\title{
Sub-eikonal corrections to scattering amplitudes at high energy
}

\author{
Giovanni Antonio Chirilli \\ Institut für Theoretische Physik, Universität Regensburg, \\ D-93040 Regensburg, Germany \\ E-mail: giovanni.chirilli@ur.de
}

ABSTRACT: Most of the progress in high-energy Quantum Chromodynamics has been obtained within the eikonal approximation and infinite Wilson-line operators. Evolution equations of Wilson lines with respect to the rapidity parameter encode the dynamics of the hadronic processes at high energy. However, even at high energy many interesting aspects of hadron dynamics are not accessible within the eikonal approximation, the spin physics being an obvious example. The higher precision reached by the experiments and the possibility to probe spin dynamics at future Electron Ion Colliders make the study of deviations from eikonal approximation especially timely. In this paper, I derive the sub-eikonal quark and gluon propagators which can serve as a starting point of studies of these effects.

Keywords: Perturbative QCD, Resummation, Scattering Amplitudes

ARXIV EPRINT: 1807.11435 


\section{Contents}

1 Introduction 1

2 Eikonal approximation for scalar and quark propagators $\quad 2$

2.1 Scalar propagator in the eikonal approximation 2

2.2 Quark propagator in eikonal approximation 5

$\begin{array}{lll}3 & \text { Sub-eikonal corrections to the scalar propagator } & 7\end{array}$

4 Sub-eikonal corrections to the quark propagator $\quad 9$

4.1 Quark propagator in the background of gluon field 9

$\begin{array}{ll}\text { 4.1.1 Symmetrizing the quark-sub-eikonal corrections } & 14\end{array}$

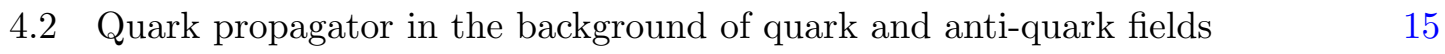

5 Sub-eikonal corrections to the gluon propagator $\quad 16$

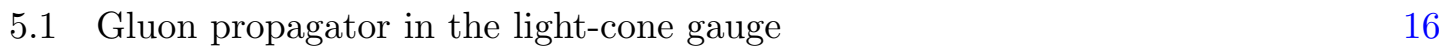

5.2 Gluon propagator in the light-cone gauge in the background of quark and anti-quark fields 20

5.3 Summing up the gluon and quark anti-quark external fields contributions 21

6 Conclusions and outlook $\quad 21$

$\begin{array}{ll}\text { A Notation } & 23\end{array}$

B An alternative type of expansion for the quark propagator $\quad 24$

C The $\left\{\hat{P}_{\bullet}, A_{*}\right\}$ term $\quad 26$

D Some calculation details of the sub-eikonal corrections to the quark $\begin{array}{ll}\text { propagator } & 27\end{array}$

E Quark and scalar propagators with one end-point in the external field $\mathbf{3 0}$

$\begin{array}{lll}\text { E.1 Scalar propagator } & 30\end{array}$

E.2 Quark propagator 32

F Gluon propagator in the background-Feynman gauge $\quad 34$

F.1 In the background of gluon field 34

F.2 In the background of quark and anti-quark fields 37

F.3 Summing up the gluon and quark anti-quark external fields contributions $\quad 42$ 


\section{Introduction}

It is well known that high-energy behavior of QCD amplitudes can be described by the evolution of relevant Wilson-line operators. The typical example is the deep inelastic scattering (DIS) at low Bjorken $x_{B}$, where the T-product of two electromagnetic currents can be approximated by a perturbative expansion in terms of coefficient functions (photon impact factors) and matrix elements of Wilson-line operators evaluated in the proton or nucleus state. The evolution equation of the Wilson-line operators with respect to the rapidity parameter provides the energy dependence of the cross section. This procedure takes the name of high-energy Operator Product Expansion (OPE) (see ref. [1] for a review on Wilson-line formalism in high-energy QCD).

The operator describing the DIS scattering amplitude is a trace of two Wilson lines. Its evolution equation with respect to the rapidity of the fields generates a hierarchy, the Balitsky-hierarchy [2], of evolution equations. The Balitsky-hierarchy is equivalent to the Jalilian-Marian-Iancu-McLerran-Weigert-Leonidov-Kovner (JIMWLK) [3-6] evolution equation thus, they are referred to as B-JIMWLK equation. The Balitsky-Kovchegov (BK) equation [7,8] is the first of the Balitsky hierarchy and it is obtained in the mean field approximation. Its linearization coincides with the BFKL $[9,10]$ equation (for a review, see ref. [11]).

The OPE in terms of composite Wilson-line operators [12] has been applied up to NLO accuracy in processes like DIS $[13,14]$ and proton-nucleus collisions $[15,16]$. In ref. [17] the analytic expression of the $\gamma^{*} \gamma^{*}$ cross section has been computed using the NLO OPE in linearized (BFKL) form.

A relevant part of the program of the proposed Electron Ion Collider [18-20] is dedicated to the study of DIS with spin. Since the high-energy OPE formalism developed so far is suitable only for unpolarized scattering processes, the extension of such formalism to high-energy spin-dynamics for Transverse Momentum Distribution (TMD) functions and $g_{1}$ structure function is in strong demand.

The high-energy OPE in terms of infinite Wilson lines is based on a semi-classical description of the process. In eikonal-approximation, the quantum free wave function of a particle propagating in a classical external field is modified by a simple phase factor in QED or gravity [21, 22], and by a path-ordered exponential in QCD [23-25]. In high-energy QCD the rapidity parameter serves as a discriminator between classical and quantum fields and the propagation of fast moving particles in an external filed is described by eikonal interactions. Unfortunately, eikonal interactions are insensitive to the spin content of the process. So, in order to bring in spin information, it is necessary to include sub-eikonal contributions. The subject of this paper is the derivation of these corrections to the quark and gluon propagator in the background of a shock-wave. These results represent the first necessary step to include spin dynamics in the high-energy OPE formalism.

In refs. [26, 27] sub-eikonal corrections to scalar and gluon propagators have been calculated in order to construct a formalism that provides evolution equations of gluon Transverse Momentum Distribution (TMD) from low to moderate $x_{B}$. In this paper, I calculate the same result for the scalar and gluon propagators but including also the 
contributions coming from the transverse gauge fields neglected in refs. [26, 27]. In addition, I compute the sub-eikonal corrections to the quark propagator. The results obtained in this paper can be used to study, for example, quark-TMDs with spin and to check results obtained in refs. [28-34].

Corrections to the eikonal formalism have been considered also in the contest of spin asymmetries in proton-nucleus collision. In ref. [35], for example, sub-eikonal corrections for the retarded gluon propagator have been calculated.

Sub-eikonal corrections have also been considered in the contest of high-energy QCD at fixed angle and resummation of threshold logarithms [36, 38].

The paper is structured as follows. In section 2.1 and in appendix A we introduce the formalism and derive the leading-eikonal scalar and quark propagator. The sub-eikonal corrections to the scalar propagator are derived in section 3. This result is a necessary step to derive the sub-eikonal corrections to the quark propagator, in section 4 , and to the gluon propagator in light-cone gauge, in section 5. In the appendix we consider a modification of the shock-wave picture, that is, we consider the case in which the particle starts or ends its propagation inside the external field. In the appendix we also include an alternative derivation of the eikonal quark propagator and we calculate the sub-eikonal corrections to the gluon propagator in background-Feynman gauge.

\section{Eikonal approximation for scalar and quark propagators}

We start our analysis with the derivation of the scalar and quark propagator in the background of a shock-wave in eikonal approximation. We will use this preliminary step in order to introduce the formalism that will be used through out the paper.

\subsection{Scalar propagator in the eikonal approximation}

The idea of the shock-wave formalism is based on the observation that high-energy regime can be reached not only rescaling the longitudinal momenta of the projectile-particle by a large parameter, but also performing a longitudinal boost of the fields generated by the target-particle. We will study the propagation of a particle in the background of a highly boosted gluon filed.

Let $p_{1}^{\mu}$ and $p_{2}^{\mu}$ be two light-cone vectors such that $p_{1 \mu} p_{2}^{\mu}=p_{1} \cdot p_{2}=\frac{s}{2}$. We assume that the projectile-particle is propagating along $p_{1}$ direction, while the target particle is moving along $p_{2}$ direction. Using the two light-cone vectors we can perform a Sudakov decomposition of the momentum $p^{\mu}=\alpha p_{1}^{\mu}+\beta p_{2}^{\mu}+p_{\perp}^{\mu}$. We also define the light-cone components $x_{\bullet}=x_{\mu} p_{1}^{\mu}=\sqrt{\frac{s}{2}} x^{-}$and $x_{*}=x_{\mu} p_{2}^{\mu}=\sqrt{\frac{s}{2}} x^{+}$with $x^{ \pm}=\frac{x^{0} \pm x^{3}}{\sqrt{2}}$. We refer the reader to the appendix A for further details on the notation that will be used.

The gauge field $A^{\mu}$ generated by the target, under a boost, gets rescaled by a large parameter $\lambda$ as follows

$$
\begin{aligned}
& A_{\bullet}\left(x_{\bullet}, x_{*}, x_{\perp}\right) \rightarrow \lambda A_{\bullet}\left(\lambda^{-1} x_{\bullet}, \lambda x_{*}, x_{\perp}\right), \\
& A_{*}\left(x_{\bullet}, x_{*}, x_{\perp}\right) \rightarrow \lambda^{-1} A_{*}\left(\lambda^{-1} x_{\bullet}, \lambda x_{*}, x_{\perp}\right), \\
& A_{\perp}\left(x_{\bullet}, x_{*}, x_{\perp}\right) \rightarrow A_{\perp}\left(\lambda^{-1} x_{\bullet}, \lambda x_{*}, x_{\perp}\right) .
\end{aligned}
$$


and the field strength tensor as

$$
\begin{aligned}
& F_{i \bullet}\left(x_{\bullet}, x_{*}, x_{\perp}\right) \rightarrow \lambda F_{i \bullet}\left(\lambda^{-1} x_{\bullet}, \lambda x_{*}, x_{\perp}\right), \\
& F_{i *}\left(x_{\bullet}, x_{*}, x_{\perp}\right) \rightarrow \lambda^{-1} F_{i *}\left(\lambda^{-1} x_{\bullet}, \lambda x_{*}, x_{\perp}\right), \\
& F_{\bullet *}\left(x_{\bullet}, x_{*}, x_{\perp}\right) \rightarrow F_{\bullet *}\left(\lambda^{-1} x_{\bullet}, \lambda x_{*}, x_{\perp}\right), \\
& F_{i j}\left(x_{\bullet}, x_{*}, x_{\perp}\right) \rightarrow F_{i j}\left(\lambda^{-1} x_{\bullet}, \lambda x_{*}, x_{\perp}\right) .
\end{aligned}
$$

In Schwinger representation the free scalar propagator is

$$
\left\langle x\left|\frac{i}{p^{2}+i \epsilon}\right| y\right\rangle=i \int d^{4} k \frac{e^{-i k \cdot(x-y)}}{k^{2}+i \epsilon},
$$

with $\langle k \mid x\rangle=e^{i x \cdot k}$. In (2.3) we used the $\hbar$-inspired notation $d^{4} k \equiv \frac{d^{4} k}{(2 \pi)^{4}}$ and $\delta^{(4)}(k)=$ $(2 \pi)^{4} \delta^{(4)}(k)$ so that, $\int d^{4} k \delta^{(4)}(k)=1$.

Because of the infinite boost, in first approximation, we can assume that the field operator $\hat{A}_{\mu}$ commutes with the $\hat{\alpha}=i \frac{\partial}{\partial x}$ operator where, as we already mentioned above, $\alpha$ is the longitudinal component along the light-cone vector $p_{1}$. The eikonal approximation is based on the observation that, the only component surviving the boost is $A_{\bullet}$ so, $\hat{P}^{2} \simeq$ $\hat{p}^{2}+2 \alpha g \hat{A}_{\bullet}$. If $A_{\mu}(x)$ is small in comparison with the typical distance $(x-y)$, we can represent the propagator as a series

$$
\begin{aligned}
\left\langle x\left|\frac{i}{\hat{P}^{2}+i \epsilon}\right| y\right\rangle \simeq & \left\langle x\left|\frac{i}{\hat{p}^{2}+i \epsilon}\right| y\right\rangle \\
& +g \int d^{4} z\left\langle x\left|\frac{i}{\hat{p}^{2}+i \epsilon}\right| z\right\rangle 2 i \alpha A_{\bullet}\left(z_{*}, z_{\perp}\right)\left\langle z\left|\frac{i}{\hat{p}^{2}+i \epsilon}\right| y\right\rangle+\ldots
\end{aligned}
$$

Alternatively, expansion (2.4) can also be expressed through Schwinger's proper time integral

$$
\begin{aligned}
\left\langle x\left|\frac{i}{\hat{P}^{2}+i \epsilon}\right| y\right\rangle= & -i \int_{0}^{+\infty} d t\left\langle x\left|e^{\left(\hat{p}^{2}+2 \alpha \hat{A} \bullet+i \epsilon\right) t}\right| y\right\rangle=-i \int_{0}^{+\infty} d t\left[\langle x| e^{i\left(\hat{p}^{2}+i \epsilon\right) t}\right. \\
& \left.+i \int_{0}^{t} d t^{\prime}\left\langle x\left|e^{i\left(t-t^{\prime}\right)\left(\hat{p}^{2}+i \epsilon\right)} 2 \alpha g \hat{A} \bullet e^{i\left(\hat{p}^{2}+i \epsilon\right) t}\right| y\right\rangle+\ldots\right]
\end{aligned}
$$

Using either (2.4) or (2.5), the expansion of the scalar propagator reduces to

$$
\begin{aligned}
\left\langle x\left|\frac{i}{\hat{P}^{2}+i \epsilon}\right| y\right\rangle= & {\left[\int_{0}^{+\infty} \frac{d \alpha}{2 \alpha} \theta\left(x_{*}-y_{*}\right)-\int_{-\infty}^{0} \frac{d \alpha}{2 \alpha} \theta\left(y_{*}-x_{*}\right)\right] e^{-i \alpha\left(x_{\bullet}-y_{\bullet}\right)} } \\
& \times\left[\left\langle x_{\perp}\left|e^{-i \frac{\hat{p}_{\perp}^{2}}{\alpha s}\left(x_{*}-y_{*}\right)}\right| y_{\perp}\right\rangle\right. \\
& \left.+\int_{y_{*}}^{x_{*}} d z_{*}\left\langle x_{\perp}\left|e^{-i \frac{\hat{p}^{2}}{\alpha s}\left(x_{*}-z_{*}\right)} i \frac{2}{s} g \hat{A}_{\bullet}\left(z_{*}\right) e^{-i \frac{\hat{p}_{\perp}^{2}}{\alpha s}\left(z_{*}-y_{*}\right)}\right| y_{\perp}\right\rangle+\ldots\right] .
\end{aligned}
$$

We are interested in the shock-wave picture relevant for high-energy scattering so, we assume that the particle starts and ends its propagation outside the interval in which the 
field strength tensor is different then zero (see figure 1). With this assumption we can rewrite expansion (2.6) as

$$
\begin{aligned}
\left\langle x\left|\frac{i}{\hat{P}^{2}+i \epsilon}\right| y\right\rangle= & {\left[\int_{0}^{+\infty} \frac{d \alpha}{2 \alpha} \theta\left(x_{*}-y_{*}\right)-\int_{-\infty}^{0} \frac{d \alpha}{2 \alpha} \theta\left(y_{*}-x_{*}\right)\right] e^{-i \alpha\left(x_{\bullet}-y_{\bullet}\right)} } \\
& \times \int d^{2} z d^{2} z^{\prime}\left\langle x_{\perp}\left|e^{-i \frac{\hat{p}_{\perp}^{2}}{\alpha s} x_{*}}\right| z_{\perp}\right\rangle \\
& \times\left\langle z_{\perp}\left|\operatorname{Pexp}\left\{i g \int_{y_{*}}^{x_{*}} d \frac{2}{s} \omega_{*} e^{i \frac{\hat{p}_{\perp}^{2}}{\alpha s} \omega_{*}} A_{\bullet}\left(\omega_{*}\right) e^{-i \frac{\hat{p}_{\perp}^{2}}{\alpha s} \omega_{*}}\right\}\right| z_{\perp}^{\prime}\right\rangle \\
& \times\left\langle z_{\perp}^{\prime}\left|e^{i \frac{\hat{p}_{\perp}^{2}}{\alpha s} y_{*}}\right| y_{\perp}\right\rangle .
\end{aligned}
$$

So far, to arrive at the propagator given in eq. (2.7), we have implemented only two consequences of the longitudinal Lorentz boost of the external field: the commutation relation $[\hat{\alpha}, \hat{A}]=0$ and the fact that the most dominant component of the gauge external field is $A_{\bullet}$. Indeed, since we are considering an infinite Lorentz boost, we can perform further approximations.

Propagator (2.7) describes the propagation, following any path from point $z_{\perp}+\frac{2}{s} x_{*} p_{1}$ to point $z_{\perp}^{\prime}+\frac{2}{s} y_{*} p_{1}$ in the external field $A^{\mu}(x)=\left(A_{\bullet}\left(x_{*}, x_{\perp}\right), 0,0\right)$, of a spinless particle. Notice that, the particle propagates between points $\left(x_{\bullet}, x_{*}, x_{\perp}\right)$ and $\left(y_{\bullet}, y_{*}, y_{\perp}\right)$, while the field strength tensor, because of the longitudinal boost, is defined within an infinitesimal interval in the longitudinal direction. In other words, $F^{\mu \nu}\left(\omega_{*}, \omega_{\perp}\right) \neq 0$ for $\omega_{*} \in\left[-\epsilon_{*}, \epsilon_{*}\right]$ with $0<\epsilon_{*} \ll 1$ and, since we are in the shock-wave case, $x_{*}, y_{*} \notin\left[-\epsilon_{*}, \epsilon_{*}\right]$.

Since we are boosting the coordinates, the longitudinal distance traveled by the particle in the external filed is rescaled under a boost as $\omega_{*} \rightarrow \frac{1}{\lambda} \omega_{*}$ while the gauge field is rescaled as $A_{\bullet} \rightarrow \lambda A_{\bullet}$ with $\lambda \gg 1$ the boost parameter. So, we can make a further approximation in eq. (2.7) and write

$$
e^{i \frac{\hat{p}_{\perp}^{2}}{\alpha s} \omega_{*}} A_{\bullet}\left(\omega_{*}\right) e^{-i \frac{\hat{p}_{\perp}^{2}}{\alpha s} \omega_{*}}=A_{\bullet}\left(\omega_{*}\right)+O\left(\lambda^{0}\right)
$$

Making use of (2.8) in propagator (2.7), we obtain

$$
\begin{aligned}
\left\langle x\left|\frac{i}{\hat{P}^{2}+i \epsilon}\right| y\right\rangle= & {\left[\int_{0}^{+\infty} \frac{d \alpha}{2 \alpha} \theta\left(x_{*}-y_{*}\right)-\int_{-\infty}^{0} \frac{d \alpha}{2 \alpha} \theta\left(y_{*}-x_{*}\right)\right] e^{-i \alpha\left(x_{\bullet}-y_{\bullet}\right)} } \\
& \times \int d^{2} z\left\langle x_{\perp}\left|e^{-i \frac{\hat{p}_{\perp}^{2}}{\alpha s} x_{*}}\right| z_{\perp}\right\rangle\left[x_{*}, y_{*}\right]_{z}\left\langle z_{\perp}\left|e^{i \frac{\hat{p}_{\perp}^{2}}{\alpha s} y_{*}}\right| y_{\perp}\right\rangle
\end{aligned}
$$

where we have defined the gauge link at fixed transverse position $z_{\perp}$ as

$$
\left[x_{*}, y_{*}\right]_{z}=\operatorname{Pexp}\left\{i g \frac{2}{s} \int_{y_{*}}^{x_{*}} d \omega_{*} A_{\bullet}\left(\omega_{*}, z_{\perp}\right)\right\} .
$$

Propagator (2.9) describes the propagation of the particle in the external field along a straight-line. Deviation from the straight-line propagation are taken into account by the higher order terms neglected in eq. (2.8). We will consider them in the next section.

In the shock wave approximation we can trade the finite gauge link with the infinite Wilson line because, under the infinite boost, the dominant component of the field strength 


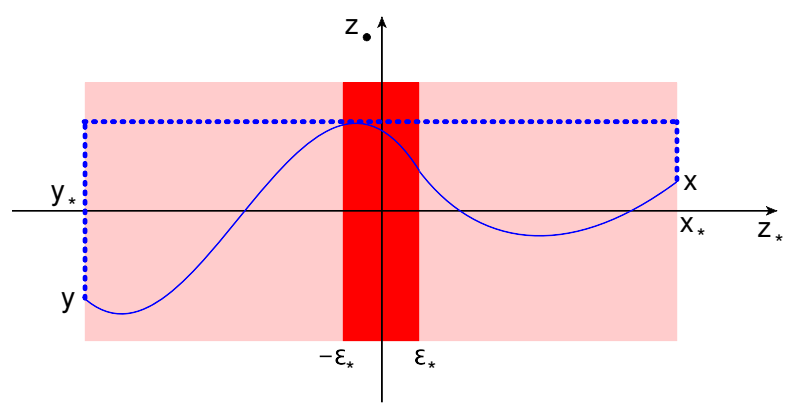

Figure 1. Particle propagating in an external gluonic field. The red strip represents the shock-wave defined in the infinitesimal interval $\left[-\epsilon_{*}, \epsilon_{*}\right]$ in which $F_{\mu \nu} \neq 0$. The light-red areas to the left and to the right of the shock-wave represent the background filed made of pure gage field. The curvy line is the particle's path from point $x$ to point $y$. The dotted lines represent the Wilson lines.

tensor, $F_{\bullet}$, has an infinitesimal thin support in $x_{*}$ coordinate. We assumed that $F_{\bullet} i$ is peaked at the origin and outside the infinitesimal interval $\left[-\epsilon_{*}, \epsilon_{*}\right], F_{\bullet}=0$ (see figure 1 ).

In the gauge rotated field $A^{\Omega}$ the gauge field outside the external field is zero so, we can trade the gauge link $\left[\epsilon_{*},-\epsilon_{*}\right]$ with the infinite Wilson line $\left[\infty p_{1},-\infty p_{1}\right]$. In this gauge we can then write

$$
\begin{aligned}
\left\langle x\left|\frac{i}{P^{2}+i \epsilon}\right| y\right\rangle= & {\left[\int_{0}^{+\infty} \frac{d \alpha}{2 \alpha} \theta\left(x_{*}-y_{*}\right)-\int_{-\infty}^{0} \frac{d \alpha}{2 \alpha} \theta\left(y_{*}-x_{*}\right)\right] e^{-i \alpha\left(x_{\bullet}-y_{\bullet}\right)} } \\
& \times \int d^{2} z\left\langle x_{\perp}\left|e^{-i \frac{\hat{p}_{\perp}^{2}}{\alpha s} x_{*}}\right| z_{\perp}\right\rangle U_{z}\left\langle z_{\perp}\left|e^{i \frac{\hat{p}_{\perp}^{2}}{\alpha s} y_{*}}\right| y_{\perp}\right\rangle,
\end{aligned}
$$

where we have defined the infinite Wilson line $U_{z}$ at fixed transverse position $z_{\perp}$ as

$$
U_{z} \equiv\left[p_{1} \infty,-p_{1} \infty\right]_{z}=\operatorname{Pexp}\left\{i g \frac{2}{s} \int_{-\infty}^{+\infty} d z_{*} A \bullet\left(\frac{2}{s} p_{1} z_{*}+z_{\perp}\right)\right\} .
$$

Notice that, because of the infinite boost, we have set $A_{\perp}^{\mu}$ component to zero, so, since it is a pure gauge, it can be restored, for example, as a transverse gauge link (see figure 1).

\subsection{Quark propagator in eikonal approximation}

We consider now the quark propagator in the eikonal approximation. From now on we work in the gauge rotated field $A^{\Omega}$. This allows us to set to zero the transverse fields at the edges of the gauge fields, i.e., $A_{i}\left(x_{*}\right)=A_{i}\left(y_{*}\right)=0$.

We proceed in the same way as in the scalar propagator case. After boosting the fields, we consider only the dominant component of the gauge fields and write

$$
\begin{aligned}
&\left\langle x\left|\frac{i}{\hat{P}+i \epsilon}\right| y\right\rangle \simeq\left\langle x\left|\hat{P} \frac{i}{\hat{P}^{2}+i g \frac{2}{s} \gamma_{\perp}^{\rho} \not p_{2} F_{\rho \bullet}+i \epsilon}\right| y\right\rangle \\
&=\left(i \not \partial^{x}+g \frac{2}{s} \not p_{2} A \bullet\left(x_{*}, x_{\perp}\right)\right) \\
& \times\left\langle x\left|\left[\frac{i}{\hat{P}^{2}+i \epsilon}-\frac{i}{\hat{P}^{2}+i \epsilon} g \frac{2}{s} \gamma_{\perp}^{\rho} \not p_{2} F_{\rho \bullet} \frac{i}{\hat{P}^{2}+i \epsilon}\right]\right| y\right\rangle,
\end{aligned}
$$


where $P^{2} \simeq p^{2}+2 g \alpha A_{\bullet}$. Notice that, because $\not p_{2} \not p_{2}=0$, there is only one term surviving the expansion in (2.13). In appendix B we will consider an alternative way of expanding the quark propagator in the external field. However, to study the sub-eikonal corrections the expansion used in this section is more convenient.

In (2.13), for each factor $\frac{1}{P^{2}+i \epsilon}$ we use the scalar propagator (2.9), and using

$$
\begin{aligned}
& \left(i \not \partial^{x}+g \frac{2}{s} \not p_{2} A_{\bullet}\left(x_{*}, x_{\perp}\right)\right) e^{-i \alpha\left(x_{\bullet}-y_{\bullet}\right)}\left\langle x_{\perp}\left|e^{-i \frac{\hat{p}_{\perp}^{2}}{\alpha s} x_{*}}\right| z_{\perp}\right\rangle \\
& =e^{-i \alpha\left(x_{\bullet}-y_{\bullet}\right)}\left\langle x_{\perp}\left|e^{-i \frac{\hat{p}_{\perp}^{2}}{\alpha s} x_{*}}\left(\frac{1}{\alpha s} \hat{p} p_{2} \hat{p}+\frac{2}{s} \not p_{2} i D_{\bullet}^{x}\right)\right| z_{\perp}\right\rangle,
\end{aligned}
$$

together with the identity

$$
i D_{\bullet}^{x}\left[x_{*}, y_{*}\right]=\left(i \partial_{\bullet}^{x}+g A_{\bullet}\left(x_{*}\right)\right)\left[x_{*}, y_{*}\right]=0
$$

we arrive at

$$
\begin{aligned}
\left\langle x\left|\frac{i}{P+i \epsilon}\right| y\right\rangle= & {\left[\int_{0}^{+\infty} \frac{d \alpha}{2 \alpha} \theta\left(x_{*}-y_{*}\right)-\int_{-\infty}^{0} \frac{d \alpha}{2 \alpha} \theta\left(y_{*}-x_{*}\right)\right] e^{-i \alpha\left(x_{\bullet}-y_{\bullet}\right)} } \\
& \times \frac{1}{\alpha s}\left\langle x_{\perp}\right| e^{-i \frac{\hat{p}_{\perp}^{2}}{\alpha s} x_{*}} \hat{\not p p} p_{2} \\
& \times\left(\hat{p}\left[x_{*}, y_{*}\right]-g \frac{2}{s} \gamma_{\perp}^{\rho} \int_{y_{*}}^{x_{*}} d \omega_{*}\left[x_{*}, \omega_{*}\right] F_{\bullet}\left[\omega_{*}, y_{*}\right]\right) e^{i \frac{\hat{p}_{\perp}^{2}}{\alpha s} y_{*}}\left|y_{\perp}\right\rangle
\end{aligned}
$$

Note that, in using eq. (2.14) we have neglected the delta function $\delta\left(x_{*}-y_{*}\right)$ coming from the differentiation of the Theta functions $\theta\left(x_{*}-y_{*}\right)$ and $\theta\left(y_{*}-x_{*}\right)$ of the scalar propagator eq. (2.9). This is because we are assuming that $x_{*} \neq y_{*}$.

Quark propagator (2.16) is in covariant gauge form. An equivalent expression of the quark propagator $(2.16)$ is

$$
\begin{aligned}
& \left\langle x\left|\frac{i}{\not P+i \epsilon}\right| y\right\rangle=\frac{1}{s}\left[\int_{0}^{+\infty} \frac{d \alpha}{2 \alpha^{2}} \theta\left(x_{*}-y_{*}\right)-\int_{-\infty}^{0} \frac{d \alpha}{2 \alpha^{2}} \theta\left(y_{*}-x_{*}\right)\right] e^{-i \alpha\left(x_{\bullet}-y_{\bullet}\right)} \\
& \times\left\langle x_{\perp}\right| e^{-i \frac{\hat{p}_{\perp}^{2}}{\alpha s} x_{*}}\left(\hat{p} p_{2}\left[x_{*}, y_{*}\right] \hat{p}-\hat{p} \not p_{2}\left[g A_{\perp}\left(x_{*}\right)\left[x_{*}, y_{*}\right]-\left[x_{*}, y_{*}\right] g A_{\perp}\left(y_{*}\right)\right]\right) \\
& \times e^{i \frac{\hat{p}_{\perp}^{2}}{\alpha s} y_{*}}\left|y_{\perp}\right\rangle \text {. }
\end{aligned}
$$

To arrive at eq. (2.17) from eq. (2.16) we have carried one of the $\hat{\not p}$ operator to the right of the gauge-link. The quark propagator in eq. (2.17) is not in gauge covariant form but it is suitable for the shock-wave picture. Indeed, in the shock-wave approximation, $A_{\perp}\left(x_{*}\right)$ and $A_{\perp}\left(y_{*}\right)$ are zero in the gauge rotated field $A^{\Omega}$ we are working in. Moreover, the gauge-link $\left[x_{*}, y_{*}\right]$, reduces to $\left[\epsilon_{*},-\epsilon_{*}\right]$. At this point we can extend the limit of integration $\epsilon_{*} \rightarrow \infty$ and $-\epsilon_{*} \rightarrow-\infty$ thus, (2.17) becomes

$$
\begin{aligned}
\left\langle x\left|\frac{i}{\not P+i \epsilon}\right| y\right\rangle= & \frac{1}{s}\left[\int_{0}^{+\infty} \frac{d \alpha}{2 \alpha^{2}} \theta\left(x_{*}-y_{*}\right)-\int_{-\infty}^{0} \frac{d \alpha}{2 \alpha^{2}} \theta\left(y_{*}-x_{*}\right)\right] e^{-i \alpha\left(x_{\bullet}-y_{\bullet}\right)} \\
& \times\left\langle x_{\perp}\left|e^{-i \frac{\hat{p}_{\perp}^{2}}{\alpha s} x_{*}} \not p\right| z_{\perp}\right\rangle \not p_{2} U_{z}\left\langle z_{\perp}\left|\not p e^{i \frac{\hat{p}_{\perp}^{2}}{\alpha s} y_{*}}\right| y_{\perp}\right\rangle .
\end{aligned}
$$


In (2.18) the shock-wave picture is now evident: we have free propagation from point $x$ to the point of interaction $z$ with the shock wave, eikonal interaction with the shock wave, which is represented by the infinite Wilson line multiplied by $\not p_{2}$, and then again free propagation from point $z$ to point $y$ (see figure 1). Propagator (2.18) is the one that has been used so far in all the results that have been obtained in the high-energy Wilson-lines formalism. In this paper we are interested in the sub-eikonal corrections to (2.18).

\section{Sub-eikonal corrections to the scalar propagator}

In the previous section, to obtain the quark propagator in the eikonal approximation, we have used the scalar propagator as intermediate step. For the sub-eikonal corrections we proceed in a similar way: we first derive the sub-eikonal correction to the scalar propagator, and then use this result as a useful intermediate step in order to get the sub-eikonal corrections to the quark propagator.

We consider a background gauge field with all components different then zero: $A_{\mu}^{c l}\left(x_{*}, x_{\perp}\right)=\left(A_{*}^{c l}\left(x_{*}, x_{\perp}\right), A_{\bullet}^{c l}\left(x_{*}, x_{\perp}\right), A_{\perp}^{c l}\left(x_{*}, x_{\perp}\right)\right)$. From now on we shall omit the superscript "cl" from the classical field. The shock-wave now has a finite width, so we have to identify the sub-dominant components of the momentum operator $\hat{P}^{2}=$ $\left\{\hat{p}_{\perp}^{\mu}, \hat{A}_{\mu}^{\perp}\right\}+\left\{\frac{2}{s} \hat{P}_{\bullet}, \hat{A}_{*}\right\}-g \hat{A}_{\perp}^{2}$. First, let us notice that we can get rid of the term $\left\{\hat{P}_{\bullet}, A_{*}\right\}$ observing that

$$
\begin{aligned}
& \left\langle x\left|\frac{1}{\hat{p}^{2}+2 \alpha g A \bullet+i \epsilon} A_{*} \hat{P}_{\bullet} \frac{1}{\hat{p}^{2}+2 \alpha g A \bullet+i \epsilon}\right| y\right\rangle \\
& =\left[-\int_{0}^{+\infty} \frac{d \alpha}{4 \alpha^{2}} \theta\left(x_{*}-y_{*}\right)+\int_{-\infty}^{0} \frac{d \alpha}{4 \alpha^{2}} \theta\left(y_{*}-x_{*}\right)\right] e^{-i \alpha\left(x_{\bullet}-y_{\bullet}\right)} \\
& \quad \times \int_{y_{*}}^{x_{*}} d \frac{2}{s} z_{*}\left\langle x_{\perp}\right| e^{-i \frac{\hat{p}_{\perp}^{2}}{\alpha s} x_{*}}\left[x_{*}, z_{*}\right]\left(A_{*}+\frac{i z_{*}}{\alpha s}\left[\hat{p}_{\perp}^{2}, A_{*}\right]\right) \\
& \quad \times\left(\frac{i z_{*}}{\alpha s}\left[\hat{p}_{\perp}^{2}, A_{\bullet}\right]+\frac{\hat{p}_{\perp}^{2}}{2 \alpha}+i D_{\bullet}^{z}\right)\left[z_{*}, y_{*}\right] e^{i \frac{\hat{p}_{\perp}^{2}}{\alpha s} y_{*}}\left|y_{\perp}\right\rangle=O\left(\lambda^{-2}\right),
\end{aligned}
$$

where in the first step we inserted $\int d^{4} z|z\rangle\langle z|=1$ and used

$$
e^{i \frac{\hat{p}_{\perp}^{2}}{\alpha s} z_{*}} A_{*} e^{-i \frac{\hat{p}_{\perp}^{2}}{\alpha s} z_{*}} \simeq A_{*}+\frac{i z_{*}}{\alpha s}\left[\hat{p}_{\perp}^{2}, A_{*}\right]
$$

and

$$
\begin{aligned}
& \left(i \partial_{\bullet}^{z}+g A_{\bullet}\left(z_{*}, z_{\perp}\right)\right)\left\langle z_{\perp}\left|e^{-i \frac{\hat{p}_{\perp}^{2}}{\alpha s} z_{*}}\left[z_{*}, y_{*}\right] e^{i \frac{\hat{p}_{\perp}^{2}}{\alpha s} y_{*}}\right| y_{\perp}\right\rangle \\
& =\left\langle z_{\perp}\left|e^{-i \frac{\hat{p}_{\perp}^{2}}{\alpha s} z_{*}}\left(\frac{i z_{*}}{\alpha s}\left[\hat{p}_{\perp}^{2}, A_{\bullet}\right]+\frac{\hat{p}_{\perp}^{2}}{2 \alpha}+i D_{\bullet}^{z}\right)\left[z_{*}, y_{*}\right] e^{i \frac{\hat{p}_{\perp}^{2}}{\alpha s} y_{*}}\right| y_{\perp}\right\rangle
\end{aligned}
$$

while in the last step we used $\left(i \partial_{\bullet}^{x}+g A_{\bullet}\left(x_{*}\right)\right)\left[x_{*}, y_{*}\right]=0$. Similarly, we can drop the term $\hat{P}_{\bullet} A_{*}$. In appendix C, following an alternative procedure, we will show that $\left\{\hat{P}_{\bullet}, A_{*}\right\}$ contributes only as sub-sub-eikonal correction. 
Let us define $\hat{O} \equiv\left\{\hat{p}_{\perp}^{\mu}, \hat{A}_{\mu}^{\perp}\right\}-g \hat{A}_{\perp}^{2}$ so, $\hat{P}^{2}=\hat{p}^{2}+2 g \alpha A_{\bullet}+g \hat{O}$. The scalar propagator in the boosted external filed can now be written as

$$
\begin{aligned}
\left\langle x\left|\frac{i}{\hat{P}^{2}+i \epsilon}\right| y\right\rangle= & \left\langle x\left|\frac{i}{\hat{p}^{2}+2 \alpha g \hat{A} \bullet+g \hat{O}+i \epsilon}\right| y\right\rangle \\
= & {\left[\int_{0}^{+\infty} \frac{d \alpha}{2 \alpha} \theta\left(x_{*}-y_{*}\right)-\int_{-\infty}^{0} \frac{d \alpha}{2 \alpha} \theta\left(y_{*}-x_{*}\right)\right] e^{-i \alpha\left(x_{\bullet}-y_{\bullet}\right)} } \\
& \times\left\langle x_{\perp}\right| e^{-i \frac{\hat{p}_{\perp}^{2}}{\alpha s} x_{*}} \operatorname{Pexp}\left\{i g \int_{y_{*}}^{x_{*}} d \frac{2}{s} \omega_{*} e^{i \frac{\hat{p}_{\perp}^{2}}{\alpha s} \omega_{*}}\left(\hat{A}_{\bullet}\left(\omega_{*}\right)+\frac{\hat{O}\left(\omega_{*}\right)}{2 \alpha}\right) e^{-i \frac{\hat{p}_{\perp}^{2}}{\alpha s} \omega_{*}}\right\} \\
& \times e^{i \frac{\hat{p}_{\perp}^{2}}{\alpha s} y_{*}}\left|y_{\perp}\right\rangle .
\end{aligned}
$$

We are interested in corrections to the eikonal propagator that go like $\frac{1}{\lambda}$, so in the following expansion it is sufficient to consider the first sub-dominant contribution which goes like $\lambda^{0}$

$$
e^{i \frac{\hat{p}_{\perp}^{2}}{\alpha s} \omega_{*}}\left(\hat{A}_{\bullet}+\frac{\hat{O}}{2 \alpha}\right) e^{-i \frac{\hat{p}_{\perp}^{2}}{\alpha s} \omega_{*}}=\hat{A}_{\bullet}+\frac{\hat{O}}{2 \alpha}+i \frac{\omega_{*}}{\alpha s}\left[\hat{p}_{\perp}^{2}, \hat{A}_{\bullet}\right]+O\left(\lambda^{-1}\right)
$$

Making use of (3.5) and of the following identity

$$
\frac{\hat{O}}{2 \alpha}+\frac{i \omega_{*}}{\alpha s}\left[\hat{p}_{\perp}^{2}, \hat{A}_{\bullet}\right]=\frac{1}{2 \alpha}\left(\left\{\hat{p}^{i}, \frac{2}{s} \omega_{*} F_{i} \bullet+\left(D \bullet \frac{2}{s} \omega_{*} \hat{A}_{i}\right)\right\}-g \hat{A}_{\perp}^{2}\right),
$$

after some algebra, we arrive at the desired expression of the scalar propagator with subeikonal corrections

$$
\begin{aligned}
\left\langle x\left|\frac{i}{\hat{P}^{2}+i \epsilon}\right| y\right\rangle= & {\left[\int_{0}^{+\infty} \frac{d \alpha}{2 \alpha} \theta\left(x_{*}-y_{*}\right)-\int_{-\infty}^{0} \frac{d \alpha}{2 \alpha} \theta\left(y_{*}-x_{*}\right)\right] e^{-i \alpha\left(x_{\bullet}-y_{\bullet}\right)} } \\
& \times\left\langle x_{\perp}\right| e^{-i \frac{\hat{p}_{\perp}^{2}}{\alpha s} x_{*}}\left\{\left[x_{*}, y_{*}\right]+\frac{i g}{2 \alpha}\left[\frac{2}{s} x_{*}\left(\left\{P_{i}, A^{i}\left(x_{*}\right)\right\}-g A_{i}\left(x_{*}\right) A^{i}\left(x_{*}\right)\right)\left[x_{*}, y_{*}\right]\right.\right. \\
& -\left[x_{*}, y_{*}\right] \frac{2}{s} y_{*}\left(\left\{P_{i}, A^{i}\left(y_{*}\right)\right\}-g A_{i}\left(y_{*}\right) A^{i}\left(y_{*}\right)\right) \\
& +\int_{y_{*}}^{x_{*}} d \frac{2}{s} \omega_{*}\left(\left\{P^{i},\left[x_{*}, \omega_{*}\right] \frac{2}{s} \omega_{*} F_{i \bullet}\left(\omega_{*}\right)\left[\omega_{*}, y_{*}\right]\right\}\right. \\
& \left.\left.\left.+g \int_{\omega_{*}}^{x_{*}} d \frac{2}{s} \omega_{*}^{\prime} \frac{2}{s}\left(\omega_{*}-\omega_{*}^{\prime}\right)\left[x_{*}, \omega_{*}^{\prime}\right] F_{\bullet}^{i}\left[\omega_{*}^{\prime}, \omega_{*}\right] F_{i}\left[\omega_{*}, y_{*}\right]\right)\right]\right\} e^{i \frac{\hat{p}_{\perp}^{2}}{\alpha s} y_{*}\left|y_{\perp}\right\rangle} \\
& +O\left(\lambda^{-2}\right) .
\end{aligned}
$$


In the shock-wave limit, the fields outside the shock-wave are pure gauge and the scalar propagator takes the following form

$$
\begin{aligned}
\left\langle x\left|\frac{i}{\hat{P}^{2}+i \epsilon}\right| y\right\rangle= & {\left[\int_{0}^{+\infty} \frac{d \alpha}{2 \alpha} \theta\left(x_{*}-y_{*}\right)-\int_{-\infty}^{0} \frac{d \alpha}{2 \alpha} \theta\left(y_{*}-x_{*}\right)\right] e^{-i \alpha\left(x_{\bullet}-y_{\bullet}\right)}\left\langle x_{\perp}\right| e^{-i \frac{\hat{p}_{\perp}^{2}}{\alpha s} x_{*}} } \\
& \times\left\{\left[\infty p_{1},-\infty p_{1}\right]+\frac{i g}{2 \alpha} \int_{-\infty}^{+\infty} \frac{2}{s} \omega_{*}\left(\left\{\hat{p}^{i},\left[\infty p_{1}, \omega_{*}\right] \frac{2}{s} \omega_{*} F_{i \bullet}\left(\omega_{*}\right)\left[\omega_{*},-\infty p_{1}\right]\right\}\right.\right. \\
& \left.\left.+g \int_{\omega_{*}}^{+\infty} d \frac{2}{s} \omega_{*}^{\prime} \frac{2}{s}\left(\omega_{*}-\omega_{*}^{\prime}\right)\left[\infty p_{1}, \omega_{*}^{\prime}\right] F^{i} \bullet\left[\omega_{*}^{\prime}, \omega_{*}\right] F_{i \bullet}\left[\omega_{*},-\infty p_{1}\right]\right)\right\} e^{i \frac{\hat{p}_{\perp}^{2}}{\alpha s} y_{*}}\left|y_{\perp}\right\rangle .
\end{aligned}
$$

Except for the terms with the transverse fields at the edges (i.e. at the points $x_{*}$ and $y_{*}$ ), propagator (3.7) coincides with the one obtained in ref. [26].

In next section we will derive, following a similar procedure, the sub-eikonal corrections to the quark propagator. To this end the result obtained in this section, eq. (3.7), will be an essential intermediate step. It is useful to notice that, the terms with transverse fields at points $x_{*}$ and $y_{*}$ in eq. (3.7) cannot be set to zero when we use the scalar propagator as intermediate step because, as we will see in next section, the points $x_{*}$ and $y_{*}$ might just be the points in the middle of the shock-wave.

\section{Sub-eikonal corrections to the quark propagator}

In this section we derive the sub-eikonal correction to the quark propagator in the background of gluon filed and quark anti-quark field. We will star with the gluon field case first.

\subsection{Quark propagator in the background of gluon field}

As already mentioned before, the sub-eikonal corrections to the scalar propagator, eq. (3.7) will be an essential intermediate step.

Our starting point is

$$
\begin{aligned}
\left\langle x\left|\frac{i}{\hat{p}+i \epsilon}\right| y\right\rangle= & \left\langle x\left|\hat{p} \frac{i}{p^{2}+2 \alpha g A_{\bullet}+g B+i \frac{2}{s} g F_{\bullet} \not p_{2} \gamma^{i}+i \epsilon}\right| y\right\rangle \\
= & \left(i \not \partial^{x}+g \frac{2}{s} A_{\bullet}\left(x_{*}, x_{\perp}\right) p_{2}+g A_{\perp}\left(x_{*}, x_{\perp}\right)\right) \\
& \times\left[\int_{0}^{+\infty} \frac{d \alpha}{2 \alpha} \theta\left(x_{*}-y_{*}\right)-\int_{-\infty}^{0} \frac{d \alpha}{2 \alpha} \theta\left(y_{*}-x_{*}\right)\right] e^{-i \alpha\left(x_{\bullet}-y_{\bullet}\right)}\left\langle x_{\perp}\right| e^{-i \frac{\hat{p}_{\perp}^{2}}{\alpha s} x_{*}} \\
& \times \operatorname{Pexp}\left\{i g \int_{y_{*}}^{x_{*}} d \frac{2}{s} \omega_{*} e^{i \frac{\hat{p}_{\perp}^{2}}{\alpha s} \omega_{*}}\left(A \cdot\left(\omega_{*}\right)+\frac{B\left(\omega_{*}\right)}{2 \alpha}+\frac{i}{2 \alpha} \frac{2}{s} F_{\bullet}\left(\omega_{*}\right) \not p_{2} \gamma^{i}\right)\right. \\
& \left.\times e^{-i \frac{\hat{p}_{\perp}^{2}}{\alpha s} \omega_{*}}\right\} e^{\hat{i}_{\perp}^{2}} y_{*}\left|y_{\perp}\right\rangle,
\end{aligned}
$$

where we have defined $B \equiv O+\frac{4}{s^{2}} g F_{\bullet *} \sigma_{* \bullet}+\frac{g}{2} F_{i j} \sigma^{i j}$ and $\sigma^{\mu \nu}=\frac{i}{2}\left(\gamma^{\mu} \gamma^{\nu}-\gamma^{\nu} \gamma^{\mu}\right)$.

Expanding again up to $\lambda^{0}$ contributions, we get

$$
\begin{aligned}
& e^{i \frac{\hat{p}_{\perp}^{2}}{\alpha s} \omega_{*}}\left(A_{\bullet}\left(\omega_{*}\right)+\frac{B\left(\omega_{*}\right)}{2 \alpha}+\frac{i}{2 \alpha} \frac{2}{s} F_{\bullet i}\left(\omega_{*}\right) \not p_{2} \gamma^{i}\right) e^{-i \frac{\hat{p}_{\perp}^{2}}{\alpha s} \omega_{*}} \\
& \quad \simeq A_{\bullet}+\frac{B}{2 \alpha}+\frac{i}{2 \alpha} \frac{2}{s} F_{\bullet} \not p_{2} \gamma^{i}+i \frac{\omega_{*}}{\alpha s}\left[p_{\perp}^{2}, A_{\bullet}\right]+i \frac{\omega_{*}}{\alpha s}\left[p_{\perp}^{2}, \frac{i}{2 \alpha} \frac{2}{s} F_{\bullet} \not p_{2} \gamma^{i}\right] .
\end{aligned}
$$


Now we have to expand the path ordered exponential of the right-hand-side of (4.2) up to the desired order. We have

$$
\begin{aligned}
\operatorname{Pexp} & \left\{i g \int_{y_{*}}^{x_{*}} d \frac{2}{s} \omega_{*} e^{i \frac{\hat{p}_{\perp}^{2}}{\alpha s} \omega_{*}}\left(A \bullet\left(\omega_{*}\right)+\frac{B\left(\omega_{*}\right)}{2 \alpha}+\frac{i}{2 \alpha} \frac{2}{s} F_{\bullet i}\left(\omega_{*}\right) p_{2} \gamma^{i}\right) e^{-i \frac{\hat{p}_{\perp}^{2}}{\alpha s} \omega_{*}}\right\} \\
= & {\left[x_{*}, y_{*}\right]+i g \int_{y_{*}}^{x_{*}} d \frac{2}{s} \omega_{*}\left[x_{*}, \omega_{*}\right]\left(\frac{O\left(\omega_{*}\right)}{2 \alpha}+i \frac{\omega_{*}}{\alpha s}\left[p_{\perp}^{2}, A_{\bullet}\right]\right)\left[\omega_{*}, y_{*}\right] } \\
& +\frac{i g}{2 \alpha} \int_{y_{*}}^{x_{*}} d \frac{2}{s} \omega_{*}\left[x_{*}, \omega_{*}\right]\left(\frac{4}{s^{2}} F_{\bullet *} \sigma_{* \bullet}+\frac{1}{2} F_{i j} \sigma^{i j}\right)\left[\omega_{*}, y_{*}\right] \\
& +i g \int_{y_{*}}^{x_{*}} d \frac{2}{s} \omega_{*}\left[x_{*}, \omega_{*}\right]\left(\frac{i}{2 \alpha} \frac{2}{s} F_{\bullet i} \not p_{2} \gamma^{i}+i \frac{\omega_{*}}{\alpha s}\left[p_{\perp}^{2}, \frac{i}{2 \alpha} \frac{2}{s} F_{\bullet} \not p_{2} \gamma^{i}\right]\right)\left[\omega_{*}, y_{*}\right] \\
& +(i g)^{2} \int_{y_{*}}^{x_{*}} d \frac{2}{s} \omega_{*} \int_{y_{*}}^{\omega_{*}} d \frac{2}{s} \omega_{*}^{\prime}\left[x_{*}, \omega_{*}\right]\left(\frac{B\left(\omega_{*}\right)}{2 \alpha}+i \frac{\omega_{*}}{\alpha s}\left[p_{\perp}^{2}, A_{\bullet}\right]\right)\left[\omega_{*}, \omega_{*}^{\prime}\right] \frac{i}{2 \alpha} \frac{2}{s} F_{\bullet i}\left(\omega_{*}^{\prime}\right) p_{2} \gamma^{i}\left[\omega_{*}^{\prime}, y_{*}\right] \\
& +(i g)^{2} \int_{y_{*}}^{x_{*}} d \frac{2}{s} \omega_{*} \int_{y_{*}}^{\omega_{*}} d \frac{2}{s} \omega_{*}^{\prime}\left[x_{*}, \omega_{*}\right] \frac{i}{2 \alpha} \frac{2}{s} F_{\bullet}\left(\omega_{*}\right) \not p_{2} \gamma^{i}\left[\omega_{*}, \omega_{*}^{\prime}\right]\left(\frac{B\left(\omega_{*}^{\prime}\right)}{2 \alpha}+i \frac{\omega_{*}^{\prime}}{\alpha s}\left[p_{\perp}^{2}, A_{\bullet}\right]\right)\left[\omega_{*}^{\prime}, y_{*}\right] \\
& +O\left(\lambda^{-2}\right) .
\end{aligned}
$$

With the help of some algebra (see appendix D for some details of the derivation), we can rewrite eq. (4.3) in a gauge invariant form as

$$
\begin{aligned}
& \operatorname{Pexp}\left\{i g \int_{y_{*}}^{x_{*}} d \frac{2}{s} \omega_{*} e^{i \frac{\hat{p}_{\perp}^{2}}{\alpha s} \omega_{*}}\left(A_{\bullet}\left(\omega_{*}\right)+\frac{B\left(\omega_{*}\right)}{2 \alpha}+\frac{i}{2 \alpha} \frac{2}{s} F_{\bullet}\left(\omega_{*}\right) \not p_{2} \gamma^{i}\right) e^{-i \frac{\hat{p}_{\perp}^{2}}{\alpha s} \omega_{*}}\right\} \\
& =\left(\left(1-\frac{1}{2 \alpha} \frac{2}{s} \not p_{2} i \mathcal{P}_{\perp}\right)\left[x_{*}, y_{*}\right]+\frac{i g}{2 \alpha} \int_{y_{*}}^{x_{*}} d \frac{2}{s} \omega_{*}\left[x_{*}, \omega_{*}\right] \mathcal{B}_{1}\left[\omega_{*}, y_{*}\right]\right) \\
& +\frac{1}{4 \alpha^{2}} \int_{y_{*}}^{x_{*}} d \frac{2}{s} z_{*}\left[i\left(\mathscr{P}_{\perp} \frac{2}{s} \not p_{2}\left[x_{*}, z_{*}\right]\right) i g \mathcal{B}_{1}\left[z_{*}, y_{*}\right]+\left[x_{*}, z_{*}\right] i g \mathcal{B}_{1}\left(i D_{\perp} \frac{2}{s} \not p_{2}\left[z_{*}, y_{*}\right]\right)\right] \\
& +\frac{i g}{2 \alpha}\left[\int _ { y _ { * } } ^ { x _ { * } } d \frac { 2 } { s } \omega _ { * } \left(\left\{p^{i},\left[x_{*}, \omega_{*}\right] \frac{2}{s} \omega_{*} F_{i \bullet}\left(\omega_{*}\right)\left[\omega_{*}, y_{*}\right]\right\}\right.\right. \\
& \left.\left.+g \int_{\omega_{*}}^{x_{*}} d \frac{2}{s} \omega_{*}^{\prime} \frac{2}{s}\left(\omega_{*}-\omega_{*}^{\prime}\right)\left[x_{*}, \omega_{*}^{\prime}\right] F^{i} \cdot\left[\omega_{*}^{\prime}, \omega_{*}\right] F_{i \bullet}\left[\omega_{*}, y_{*}\right]\right)\right]-\frac{i g}{4 \alpha^{2}} \gamma^{j} \frac{2}{s} \not p_{2} \int_{y_{*}}^{x_{*}} d \frac{2}{s} \omega_{*} \frac{2}{s} \omega_{*} \\
& \times\left[\left(i \mathfrak{D}^{i}\left[x_{*}, \omega_{*}\right]\right)\left(i D_{i} F_{j \bullet}\right)\left[\omega_{*}, y_{*}\right]-\left[x_{*}, \omega_{*}\right]\left(i D^{i} F_{j \bullet}\right)\left(i \mathfrak{D}_{i}\left[\omega_{*}, y_{*}\right]\right)-\left\{p^{i},\left[x_{*}, \omega_{*}\right]\left(i D_{i} F_{j \bullet}\right)\left[\omega_{*}, y_{*}\right]\right\}\right] \\
& +\frac{g}{4 \alpha^{2}} \frac{2}{s} \not p_{2} \int_{y_{*}}^{x_{*}} d \frac{2}{s} \omega_{*}\left[\frac{2}{s} \omega_{*}\left(\mathscr{D}_{\perp}\left[x_{*}, \omega_{*}\right]\right) F_{i \bullet}\left(i \mathfrak{D}^{i}\left[\omega_{*}, y_{*}\right]\right)-\frac{2}{s} \omega_{*}\left(i \mathfrak{D}^{i}\left[x_{*}, \omega_{*}\right]\right) F_{i \bullet}\left(\mathfrak{P}_{\perp}\left[\omega_{*}, y_{*}\right]\right)\right. \\
& \left.+\left\{p^{i},\left[x_{*}, \omega_{*}\right] \frac{2}{s} \omega_{*} F_{i \bullet}\left(\mathcal{D}_{\perp}\left[\omega_{*}, y_{*}\right]\right)\right\}+\left\{p^{i}, \mathscr{D}_{\perp}\left[x_{*}, \omega_{*}\right]\right) \frac{2}{s} \omega_{*} F_{i \bullet}\left[\omega_{*}, y_{*}\right]\right\} \\
& \left.+\left[x_{*}, \omega_{*}\right] \frac{2}{s} \omega_{*} F_{i \bullet} i \mathfrak{D}^{i}\left(\mathscr{D}_{\perp}\left[\omega_{*}, y_{*}\right]\right)-\left(i \mathfrak{D}^{i}\left(\mathscr{D}_{\perp}\left[x_{*}, \omega_{*}\right]\right)\right) \frac{2}{s} \omega_{*} F_{i \bullet}\left[\omega_{*}, y_{*}\right]\right] \\
& +O\left(\lambda^{-2}\right) \text {, }
\end{aligned}
$$

where we have defined $\mathcal{B}_{1} \equiv \frac{4}{s^{2}} F_{\bullet *} \sigma_{* \bullet}+\frac{1}{2} F_{i j} \sigma^{i j}$. 
To continue our analysis we observe that

$$
\begin{aligned}
\left(i \not \partial^{x}\right. & \left.+g \frac{2}{s} \not p_{2} A_{\bullet}\left(x_{*}, x_{\perp}\right)+g A_{\perp}\left(x_{*}, x_{\perp}\right)\right) e^{-i \alpha\left(x_{\bullet}-y_{\bullet}\right)}\left\langle x_{\perp}\left|e^{-i \frac{\hat{p}_{\perp}^{2}}{\alpha s} x_{*}}\right| z_{\perp}\right\rangle \\
= & e^{-i \alpha\left(x_{\bullet}-y_{\bullet}\right)}\left\langle x_{\perp}\right| e^{-i \frac{\hat{p}_{\perp}^{2}}{\alpha s} x_{*}} \\
& \times\left(\frac{1}{\alpha s} \not p p_{2} \not p+i \frac{2}{s} \not p_{2} D_{\bullet}^{x}+\frac{i x_{*}}{\alpha s}\left[p_{\perp}^{2}, g \frac{2}{s} \not p_{2} A_{\bullet}\left(x_{*}\right)\right]+g A_{\perp}\left(x_{*}\right)\right)\left|z_{\perp}\right\rangle,
\end{aligned}
$$

and note that $i\left[p_{\perp}^{2}, g A_{\bullet}\left(x_{*}\right)\right]=g\left\{p^{i}, F_{i \bullet}\left(x_{*}\right)+D_{\bullet} A_{i}\left(x_{*}\right)\right\}$. The field strength tensor $F_{i \bullet}\left(x_{*}\right)=0$ since $x_{*}$ is outside the shock-wave (see figure 1). Similarly, we can set all the transverse fields at the edges of the gauge-link (outside the shock-wave) to zero since they are pure gauge. So, (4.5) reduces to

$$
\begin{gathered}
\left(i \not \partial^{x}+g \frac{2}{s} \not p_{2} A_{\bullet}\left(x_{*}, x_{\perp}\right)+g A_{\perp}\left(x_{*}, x_{\perp}\right)\right) e^{-i \alpha\left(x_{\bullet}-y_{\bullet}\right)}\left\langle x_{\perp}\left|e^{-i \frac{\hat{p}_{\perp}^{2}}{\alpha s} x_{*}}\right| z_{\perp}\right\rangle \\
=e^{-i \alpha\left(x_{\bullet}-y_{\bullet}\right)}\left\langle x_{\perp}\left|e^{-i \frac{\hat{p}_{\perp}^{2}}{\alpha s} x_{*}}\left(\frac{1}{\alpha s} \not p p_{2} \not p+i \frac{2}{s} \not p_{2} D_{\bullet}^{x}\right)\right| z_{\perp}\right\rangle .
\end{gathered}
$$

Using (4.6) and the identity $i D_{\bullet}^{x}\left[x_{*}, y_{*}\right]=0$, we arrive at the following expression for the quark propagator

$$
\begin{aligned}
& \left\langle x\left|\frac{i}{\hat{p}+i \epsilon}\right| y\right\rangle \\
& =\left[\int_{0}^{+\infty} \frac{d \alpha}{2 \alpha} \theta\left(x_{*}-y_{*}\right)-\int_{-\infty}^{0} \frac{d \alpha}{2 \alpha} \theta\left(y_{*}-x_{*}\right)\right] e^{-i \alpha\left(x_{\bullet}-y_{\bullet}\right)} \frac{1}{\alpha s}\left\langle x_{\perp}\right| e^{-i \frac{\hat{p}_{\perp}^{2}}{\alpha s} x_{*}} \\
& \times\left\{\not p p_{2} \not p\left(1-\frac{1}{2 \alpha} \frac{2}{s} \not p_{2} i \mathfrak{D}_{\perp}\right)\left[x_{*}, y_{*}\right]+\frac{i g}{2 \alpha} \int_{y_{*}}^{x_{*}} d \frac{2}{s} z_{*} \not p p p_{2} \not p\left[x_{*}, z_{*}\right] \mathcal{B}_{1}\left[z_{*}, y_{*}\right]\right. \\
& +\frac{1}{4 \alpha^{2}} \frac{2}{s} \int_{y_{*}}^{x_{*}} d \frac{2}{s} z_{*} \not p p_{2} \not p\left[\left(i \mathfrak{P}_{\perp} \not p_{2}\left[x_{*}, z_{*}\right]\right) i g \mathcal{B}_{1}\left[z_{*}, y_{*}\right]+\left[x_{*}, z_{*}\right] i g \mathcal{B}_{1}\left(i \mathfrak{P}_{\perp} \not p_{2}\left[z_{*}, y_{*}\right]\right)\right] \\
& +\frac{i g}{2 \alpha} \int_{y_{*}}^{x_{*}} d \frac{2}{s} \omega_{*} \not p p_{2} \not p\left(\left\{P^{i},\left[x_{*}, \omega_{*}\right] \frac{2}{s} \omega_{*} F_{i \bullet}\left(\omega_{*}\right)\left[\omega_{*}, y_{*}\right]\right\}\right. \\
& \left.+g \int_{\omega_{*}}^{x_{*}} d \frac{2}{s} \omega_{*}^{\prime} \frac{2}{s}\left(\omega_{*}-\omega_{*}^{\prime}\right)\left[x_{*}, \omega_{*}^{\prime}\right] F^{i} \bullet\left[\omega_{*}^{\prime}, \omega_{*}\right] F_{i \bullet}\left[\omega_{*}, y_{*}\right]\right) \\
& +\frac{i g}{2 \alpha} \not p \gamma^{j} \not p_{2} \int_{y_{*}}^{x_{*}} d \frac{2}{s} z_{*} \frac{2}{s} z_{*}\left[\left\{P^{i},\left[x_{*}, z_{*}\right]\left(i D_{i} F_{j \bullet}\right)\left[z_{*}, y_{*}\right]\right\}-\left(i \mathfrak{D}^{i}\left[x_{*}, z_{*}\right]\right)\left(i D_{i} F_{j \bullet}\right)\left[z_{*}, y_{*}\right]\right. \\
& \left.+\left[x_{*}, z_{*}\right]\left(i D_{i} F_{j \bullet}\right)\left(i \mathfrak{D}^{i}\left[z_{*}, y_{*}\right]\right)\right]+\frac{i g}{2 \alpha} \not p p_{2} \int_{y_{*}}^{x_{*}} d \frac{2}{s} z_{*} \frac{2}{s} z_{*}\left[\left(i \mathfrak{D}^{i}\left(i \mathcal{D}_{\perp}\left[x_{*}, z_{*}\right]\right)\right) F_{i \bullet}\left[z_{*}, y_{*}\right]\right. \\
& -\left[x_{*}, z_{*}\right] F_{i \bullet}\left(i \mathfrak{D}^{i}\left(i \mathscr{D}_{\perp}\left[z_{*}, y_{*}\right]\right)\right)+\left(i \mathfrak{D}^{i}\left[x_{*}, z_{*}\right]\right) F_{i \bullet}\left(i \mathfrak{D}_{\perp}\left[z_{*}, y_{*}\right]\right) \\
& -\left\{P^{i},\left(i \mathscr{D}_{\perp}\left[x_{*}, z_{*}\right]\right) F_{i \bullet}\left[z_{*}, y_{*}\right]\right\}-\left\{P^{i},\left[x_{*}, z_{*}\right] F_{i \bullet}\left(i \mathscr{D}_{\perp}\left[z_{*}, y_{*}\right]\right)\right\} \\
& \left.\left.-\left(i \mathfrak{D}_{\perp}\left[x_{*}, z_{*}\right]\right) F^{i} \cdot\left(i \mathfrak{D}_{i}\left[z_{*}, y_{*}\right]\right)\right]\right\} e^{i \frac{\hat{p}_{\perp}^{2}}{\alpha s} y_{*}}\left|y_{\perp}\right\rangle \text {. }
\end{aligned}
$$

In order to have $\hat{\not}$ to the left and to the right of the gauge link, which is suitable for the shock-wave picture, we will perform similar steps we performed in going from eq. (2.16) 
to eq. (2.17), and set again to zero the fields $A_{i}$ at the point $x_{*}$ and $y_{*}$. To this end, we notice that

$$
\begin{aligned}
& \frac{i g}{2 \alpha} \int_{y_{*}}^{x_{*}} d \frac{2}{s} \omega_{*} \hat{p p} p_{2} \hat{\not p}\left(\left\{P^{i},\left[x_{*}, \omega_{*}\right] \frac{2}{s} \omega_{*} F_{i \bullet}\left(\omega_{*}\right)\left[\omega_{*}, y_{*}\right]\right\}\right. \\
& \left.+g \int_{\omega_{*}}^{x_{*}} d \frac{2}{s} \omega_{*}^{\prime} \frac{2}{s}\left(\omega_{*}-\omega_{*}^{\prime}\right)\left[x_{*}, \omega_{*}^{\prime}\right] F^{i} \bullet\left[\omega_{*}^{\prime}, \omega_{*}\right] F_{i} \bullet\left[\omega_{*}, y_{*}\right]\right) \\
& +\frac{i g}{2 \alpha} \hat{p} \gamma^{j} \not p_{2} \int_{y_{*}}^{x_{*}} d \frac{2}{s} z_{*} \frac{2}{s} z_{*}\left[\left\{P^{i},\left[x_{*}, z_{*}\right]\left(i D_{i} F_{j \bullet}\right)\left[z_{*}, y_{*}\right]\right\}-\left(i \mathfrak{D}^{i}\left[x_{*}, z_{*}\right]\right) i \mathfrak{D}_{i} F_{j \bullet}\left[z_{*}, y_{*}\right]\right. \\
& \left.+\left[x_{*}, z_{*}\right]\left(i D_{i} F_{j \bullet}\right)\left(i \mathfrak{D}^{i}\left[z_{*}, y_{*}\right]\right)\right]+\frac{i g}{2 \alpha} \hat{p} p_{2} \int_{y_{*}}^{x_{*}} d \frac{2}{s} z_{*} \frac{2}{s} z_{*}\left[\left(i \mathfrak{D}^{i}\left(i \mathfrak{D}_{\perp}\left[x_{*}, z_{*}\right]\right)\right) F_{i \bullet}\left[z_{*}, y_{*}\right]\right. \\
& -\left[x_{*}, z_{*}\right] F_{i \bullet}\left(i \mathfrak{D}^{i}\left(i \mathfrak{D}_{\perp}\left[z_{*}, y_{*}\right]\right)\right)+\left(i \mathfrak{D}^{i}\left[x_{*}, z_{*}\right]\right) F_{i \bullet}\left(i \mathfrak{D}_{\perp}\left[z_{*}, y_{*}\right]\right) \\
& -\left(i \mathfrak{D}_{\perp}\left[x_{*}, z_{*}\right]\right) F^{i} \bullet\left(i \mathfrak{D}_{i}\left[z_{*}, y_{*}\right]\right)-\left\{P^{i},\left(i \mathfrak{D}_{\perp}\left[x_{*}, z_{*}\right]\right) F_{i \bullet}\left[z_{*}, y_{*}\right]\right\} \\
& \left.-\left\{P^{i},\left[x_{*}, z_{*}\right] F_{i \bullet}\left(i \mathcal{P}_{\perp}\left[z_{*}, y_{*}\right]\right)\right\}\right] \\
& =\frac{i g}{2 \alpha} \int_{y_{*}}^{x_{*}} d \frac{2}{s} \omega_{*} \hat{p p} p_{2}\left(\frac{2}{s} \omega_{*}\left\{P_{i},\left[x_{*}, \omega_{*}\right] F^{i} \cdot\left[\omega_{*}, y_{*}\right]\right\}\right. \\
& \left.+g \int_{\omega_{*}}^{x_{*}} d \frac{2}{s} \omega_{*}^{\prime} \frac{2}{s}\left(\omega_{*}-\omega_{*}^{\prime}\right)\left[x_{*}, \omega_{*}^{\prime}\right] F^{i} \cdot\left[\omega_{*}^{\prime}, \omega_{*}\right] F_{i \bullet}\left[\omega_{*}, y_{*}\right]\right) \hat{p},
\end{aligned}
$$

where we have used $F_{i j}\left(x_{*}\right)=F_{i j}\left(y_{*}\right)=0$, as points $x_{*}$ and $y_{*}$ are outside the shock-wave (see figure 1). To arrive at eq. (4.8) we have used eqs. (A.5), (A.6), (A.7), and the fact that $\hat{\alpha}$ commutes with all fields.

Using eq. (4.8) in eq. (4.7) we arrive at

$$
\begin{aligned}
\left\langle x\left|\frac{i}{\hat{p}+i \epsilon}\right| y\right\rangle= & {\left[\int_{0}^{+\infty} \frac{d \alpha}{2 \alpha} \theta\left(x_{*}-y_{*}\right)-\int_{-\infty}^{0} \frac{d \alpha}{2 \alpha} \theta\left(y_{*}-x_{*}\right)\right] e^{-i \alpha\left(x_{\bullet}-y_{\bullet}\right)} \frac{1}{\alpha s}\left\langle x_{\perp}\right| e^{-i \frac{\hat{p}_{\perp}^{2}}{\alpha s} x_{*}} } \\
& \times\left\{\hat{\not p} p_{2}\left[x_{*}, y_{*}\right] \hat{\not p}+\frac{i g}{2 \alpha} \int_{y_{*}}^{x_{*}} d \frac{2}{s} z_{*} \hat{\not p} p_{2} \hat{\not p}\left[x_{*}, z_{*}\right] \mathcal{B}_{1}\left[z_{*}, y_{*}\right]\right. \\
& +\frac{1}{4 \alpha^{2}} \frac{2}{s} \int_{y_{*}}^{x_{*}} d \frac{2}{s} z_{*} \hat{\not p} p_{2} \hat{p}\left[\left(i \mathcal{D}_{\perp} \not p_{2}\left[x_{*}, z_{*}\right]\right) i g \mathcal{B}_{1}\left[z_{*}, y_{*}\right]+\left[x_{*}, z_{*}\right] i g \mathcal{B}_{1}\left(i \mathfrak{D}_{\perp} \not p_{2}\left[z_{*}, y_{*}\right]\right)\right] \\
& +\frac{i g}{2 \alpha} \int_{y_{*}}^{x_{*}} d \frac{2}{s} \omega_{*} \hat{\not p} p_{2}\left(\left\{p^{i},\left[x_{*}, \omega_{*}\right] \frac{2}{s} \omega_{*} F_{i \bullet}\left(\omega_{*}\right)\left[\omega_{*}, y_{*}\right]\right\}\right. \\
& \left.\left.+g \int_{\omega_{*}}^{x_{*}} d \frac{2}{s} \omega_{*}^{\prime} \frac{2}{s}\left(\omega_{*}-\omega_{*}^{\prime}\right)\left[x_{*}, \omega_{*}^{\prime}\right] F_{\bullet}^{i}\left[\omega_{*}^{\prime}, \omega_{*}\right] F_{i}\left[\omega_{*}, y_{*}\right]\right) \hat{p}\right\} e^{i \frac{\hat{p}_{\perp}^{2}}{\alpha s} y_{*}\left|y_{\perp}\right\rangle .}
\end{aligned}
$$

We now use the following results

$$
\begin{aligned}
& \frac{i g}{2 \alpha} \int_{y_{*}}^{x_{*}} d \frac{2}{s} z_{*} \hat{\not p} p_{2} \hat{\not p}\left[x_{*}, z_{*}\right] \frac{1}{2} F_{i j} \sigma^{i j}\left[z_{*}, y_{*}\right] \\
& +\frac{1}{4 \alpha^{2}} \frac{2}{s} \int_{y_{*}}^{x_{*}} d \frac{2}{s} z_{*} \hat{\not p p} p_{2} \hat{\not p}\left[\left(i \not D_{\perp} \not p_{2}\left[x_{*}, z_{*}\right]\right) i g \frac{1}{2} F_{i j} \sigma^{i j}\left[z_{*}, y_{*}\right]+\left[x_{*}, z_{*}\right] i g \frac{1}{2} F_{i j} \sigma^{i j}\left(i \mathfrak{D}_{\perp} \not p_{2}\left[z_{*}, y_{*}\right]\right)\right] \\
& =\frac{i g}{2 \alpha} \int_{y_{*}}^{x_{*}} d \frac{2}{s} z_{*} \hat{\not p h} p_{2}\left[\left[x_{*}, z_{*}\right] \frac{1}{2} F_{i j} \sigma^{i j}\left[z_{*}, y_{*}\right] \hat{p}+\left[x_{*}, z_{*}\right] \frac{1}{4}\left(i \mathfrak{D}_{k} F_{i j}\right)\left\{\sigma^{i j}, \gamma^{k}\right\}\left[z_{*}, y_{*}\right]\right. \\
& \left.\quad+\left\{\hat{p}_{k},\left[x_{*}, z_{*}\right] i F_{k j} \gamma^{j}\left[z_{*}, y_{*}\right]\right\}+\left[x_{*}, z_{*}\right] i F_{k j} \gamma^{j}\left(i \mathfrak{D}^{k}\left[z_{*}, y_{*}\right]\right)-\left(i \mathfrak{D}^{k}\left[x_{*}, z_{*}\right]\right) i F_{k j} \gamma^{j}\left[z_{*}, y_{*}\right]\right],
\end{aligned}
$$


and

$$
\begin{aligned}
& \frac{i g}{2 \alpha} \int_{y_{*}}^{x_{*}} d \frac{2}{s} z_{*} \hat{p p} p_{2} \hat{\not p}\left[x_{*}, z_{*}\right] \frac{4}{s^{2}} F_{\bullet *} \sigma_{* \bullet}\left[z_{*}, y_{*}\right] \\
& +\frac{1}{4 \alpha^{2}} \frac{2}{s} \int_{y_{*}}^{x_{*}} d \frac{2}{s} z_{*} \hat{p} p_{2} \hat{p}\left[\left(i \not D_{\perp} \not p_{2}\left[x_{*}, z_{*}\right]\right) i g \frac{4}{s^{2}} F_{\bullet *} \sigma_{* \bullet}\left[z_{*}, y_{*}\right]+\left[x_{*}, z_{*}\right] i g \frac{4}{s^{2}} F_{\bullet *} \sigma_{* \bullet}\left(i \mathfrak{D}_{\perp} \not p_{2}\left[z_{*}, y_{*}\right]\right)\right] \\
& =\frac{i g}{2 \alpha} \int_{y_{*}}^{x_{*}} d \frac{2}{s} z_{*} i \frac{s}{2} \hat{\not p} p_{2}\left[\left(\hat{\alpha} \not p_{1}-\hat{p}_{\perp}\right)\left[x_{*}, z_{*}\right] \frac{4}{s^{2}} F_{\bullet *}\left[z_{*}, y_{*}\right]\right. \\
& \left.+\left(i \mathscr{D}_{\perp}\left[x_{*}, z_{*}\right]\right) \frac{4}{s^{2}} F_{\bullet *}\left[z_{*}, y_{*}\right]-\left[x_{*}, z_{*}\right] \frac{4}{s^{2}} F_{\bullet *}\left(i \mathcal{P}_{\perp}\left[z_{*}, y_{*}\right]\right)\right],
\end{aligned}
$$

and eq. (4.9) becomes

$$
\begin{aligned}
\langle x| & \frac{i}{\hat{p}+i \epsilon}|y\rangle \\
= & {\left[\int_{0}^{+\infty} \frac{d \alpha}{2 \alpha} \theta\left(x_{*}-y_{*}\right)-\int_{-\infty}^{0} \frac{d \alpha}{2 \alpha} \theta\left(y_{*}-x_{*}\right)\right] e^{-i \alpha\left(x_{\bullet}-y_{\bullet}\right)} \frac{1}{\alpha s}\left\langle x_{\perp}\right| e^{-i \frac{\hat{p}_{\perp}^{2}}{\alpha s} x_{*}} } \\
& \times\left\{\hat{p} p_{2}\left[x_{*}, y_{*}\right] \hat{p}+\frac{i g}{2 \alpha} \int_{y_{*}}^{x_{*}} d \frac{2}{s} \omega_{*} \hat{\not p p} p_{2}\left(\left\{p^{i},\left[x_{*}, \omega_{*}\right] \frac{2}{s} \omega_{*} F_{i \bullet}\left(\omega_{*}\right)\left[\omega_{*}, y_{*}\right]\right\}\right.\right. \\
& \left.+\left[x_{*}, \omega_{*}\right] \frac{1}{2} F_{i j} \sigma^{i j}\left[\omega_{*}, y_{*}\right]+g \int_{\omega_{*}}^{x_{*}} d \frac{2}{s} \omega_{*}^{\prime} \frac{2}{s}\left(\omega_{*}-\omega_{*}^{\prime}\right)\left[x_{*}, \omega_{*}^{\prime}\right] F_{\bullet}^{i}\left[\omega_{*}^{\prime}, \omega_{*}\right] F_{i \bullet}\left[\omega_{*}, y_{*}\right]\right) \hat{p} \\
& +\frac{i g}{2 \alpha} \int_{y_{*}}^{x_{*}} d \frac{2}{s} \omega_{*} \hat{\not p} p_{2}\left[\left[x_{*}, \omega_{*}\right] \frac{i}{4}\left\{\left(i \not D_{\perp} F_{i j}\right), \gamma^{i} \gamma^{j}\right\}\left[\omega_{*}, y_{*}\right]+\left\{\hat{p}^{k},\left[x_{*}, \omega_{*}\right] i F_{k j} \gamma^{j}\left[\omega_{*}, y_{*}\right]\right\}\right. \\
& +\left[x_{*}, \omega_{*}\right] i F_{k j} \gamma^{j}\left(i \mathfrak{D}^{k}\left[\omega_{*}, y_{*}\right]\right)-\left(i \mathfrak{D}^{k}\left[x_{*}, \omega_{*}\right]\right) i F_{k j} \gamma^{j}\left[\omega_{*}, y_{*}\right] \\
& +\left(\hat{\alpha} p_{1}-\hat{p}_{\perp}\right)\left[x_{*}, \omega_{*}\right] i \frac{2}{s} F_{\bullet}\left[\omega_{*}, y_{*}\right]+\left(i \mathfrak{P}_{\perp}\left[x_{*}, \omega_{*}\right]\right) i \frac{2}{s} F_{\bullet *}\left[\omega_{*}, y_{*}\right] \\
& \left.\left.-\left[x_{*}, \omega_{*}\right] i \frac{2}{s} F_{\bullet *}\left(i \mathfrak{D}_{\perp}\left[\omega_{*}, y_{*}\right]\right)\right]\right\} e^{i \frac{\hat{p}_{\perp}^{2}}{\alpha s} y_{*}\left|y_{\perp}\right\rangle .}
\end{aligned}
$$

To underline the structure of result (4.12), we define the following two operators

$$
\begin{aligned}
\hat{\mathcal{O}}_{1}\left(x_{*}, y_{*} ; p_{\perp}\right)= & \frac{i g}{2 \alpha} \int_{y_{*}}^{x_{*}} d \frac{2}{s} \omega_{*}\left(\left[x_{*}, \omega_{*}\right] \frac{1}{2} \sigma^{i j} F_{i j}\left[\omega_{*}, y_{*}\right]+\left\{\hat{p}^{i},\left[x_{*}, \omega_{*}\right] \frac{2}{s} \omega_{*} F_{i \bullet}\left(\omega_{*}\right)\left[\omega_{*}, y_{*}\right]\right\}\right. \\
& \left.+g \int_{\omega_{*}}^{x_{*}} d \frac{2}{s} \omega_{*}^{\prime} \frac{2}{s}\left(\omega_{*}-\omega_{*}^{\prime}\right)\left[x_{*}, \omega_{*}^{\prime}\right] F^{i} \bullet\left[\omega_{*}^{\prime}, \omega_{*}\right] F_{i \bullet}\left[\omega_{*}, y_{*}\right]\right)
\end{aligned}
$$

and

$$
\begin{aligned}
\hat{\mathcal{O}}_{2}\left(x_{*}, y_{*} ; p_{\perp}\right)= & \frac{i g}{2 \alpha} \int_{y_{*}}^{x_{*}} \frac{2}{s} \omega_{*}\left[\left[x_{*}, \omega_{*}\right] \frac{i}{4}\left\{\left(i \not D_{\perp} F_{i j}\right), \gamma^{i} \gamma^{j}\right\}\left[\omega_{*}, y_{*}\right]+\left\{\hat{p}^{k},\left[x_{*}, \omega_{*}\right] i F_{k j} \gamma^{j}\left[\omega_{*}, y_{*}\right]\right\}\right. \\
& +\left[x_{*}, \omega_{*}\right] i F_{k j} \gamma^{j}\left(i \mathfrak{D}^{k}\left[\omega_{*}, y_{*}\right]\right)-\left(i \mathfrak{D}^{k}\left[x_{*}, \omega_{*}\right]\right) i F_{k j} \gamma^{j}\left[\omega_{*}, y_{*}\right] \\
& -\left[x_{*}, \omega_{*}\right] i \frac{2}{s} F_{\bullet *}\left(i \mathfrak{D}_{\perp}\left[\omega_{*}, y_{*}\right]\right)+\left(i \mathfrak{D}_{\perp}\left[x_{*}, \omega_{*}\right]\right) i \frac{2}{s} F_{\bullet *}\left[\omega_{*}, y_{*}\right] \\
& \left.+\left(\hat{\alpha} \phi_{1}-\hat{p}_{\perp}\right)\left[x_{*}, \omega_{*}\right] i \frac{2}{s} F_{\bullet *}\left[\omega_{*}, y_{*}\right]\right]
\end{aligned}
$$


where

$\frac{i g}{2 \alpha} \int_{y_{*}}^{x_{*}} d \frac{2}{s} \omega_{*}\left[\left[x_{*}, \omega_{*}\right] i F_{k j} \gamma^{j}\left(i \mathfrak{D}^{k}\left[\omega_{*}, y_{*}\right]\right)-\left(i \mathfrak{D}^{k}\left[x_{*}, \omega_{*}\right]\right) i F_{k j} \gamma^{j}\left[\omega_{*}, y_{*}\right]\right]$
$=\frac{i g}{2 \alpha} \int_{y_{*}}^{x_{*}} d \frac{2}{s} \omega_{*} \int_{\omega_{*}}^{x_{*}} d \frac{2}{s} \omega_{*}^{\prime}\left[\left[x_{*}, \omega_{*}^{\prime}\right] g F^{k}\left[\omega_{*}^{\prime}, \omega_{*}\right] i F_{k j} \gamma^{j}\left[\omega_{*}, y_{*}\right]-\left[x_{*}, \omega_{*}^{\prime}\right] i F_{k j} \gamma^{j}\left[\omega_{*}^{\prime}, \omega_{*}\right] g F^{k}\left[\omega_{*}, y_{*}\right]\right]$

and

$$
\begin{gathered}
\frac{i g}{2 \alpha} \int_{y_{*}}^{x_{*}} d \frac{2}{s} \omega_{*}\left[-\left[x_{*}, \omega_{*}\right] i \frac{2}{s} F_{\bullet *}\left(i \mathcal{D}_{\perp}\left[\omega_{*}, y_{*}\right]\right)+\left(i \mathcal{D}_{\perp}\left[x_{*}, \omega_{*}\right]\right) i \frac{2}{s} F_{\bullet *}\left[\omega_{*}, y_{*}\right]\right] \\
=\frac{i g}{2 \alpha} \int_{y_{*}}^{x_{*}} d \frac{2}{s} \omega_{*} \int_{\omega_{*}}^{x_{*}} d \frac{2}{s} \omega_{*}^{\prime}\left[\left[x_{*}, \omega_{*}^{\prime}\right] i \frac{2}{s} F_{\bullet *}\left[\omega_{*}^{\prime}, \omega_{*}\right] \gamma^{k} g F_{k \bullet}\left[\omega_{*}, y_{*}\right]\right. \\
\left.-\left[x_{*}, \omega_{*}^{\prime}\right] \gamma^{k} g F_{k \bullet}\left[\omega_{*}^{\prime}, \omega_{*}\right] i \frac{2}{s} F_{\bullet *}\left[\omega_{*}, y_{*}\right]\right]
\end{gathered}
$$

and result (4.9) becomes

$$
\begin{aligned}
\left\langle x\left|\frac{i}{\hat{p}+i \epsilon}\right| y\right\rangle= & {\left[\int_{0}^{+\infty} \frac{d \alpha}{2 \alpha} \theta\left(x_{*}-y_{*}\right)-\int_{-\infty}^{0} \frac{d \alpha}{2 \alpha} \theta\left(y_{*}-x_{*}\right)\right] e^{-i \alpha\left(x_{\bullet}-y_{\bullet}\right)} \frac{1}{\alpha s}\left\langle x_{\perp}\right| e^{-i \frac{\hat{p}_{\perp}^{2}}{\alpha s} x_{*}} } \\
& \times\left\{\hat{\not p} p_{2}\left[x_{*}, y_{*}\right] \hat{\not p}+\hat{\not p} p_{2} \hat{\mathcal{O}}_{1}\left(x_{*}, y_{*} ; p_{\perp}\right) \hat{\not p}+\hat{\not p} p_{2} \hat{\mathcal{O}}_{2}\left(x_{*}, y_{*} ; p_{\perp}\right)\right\} e^{i \frac{\hat{p}_{\perp}^{2}}{\alpha s} y_{*}}\left|y_{\perp}\right\rangle \\
& +O\left(\lambda^{-2}\right) .
\end{aligned}
$$

Equation (4.17) is the final result for the quark propagator with sub-eikonal corrections in a non symmetric form. In next section we will rewrite it in a symmetric form with respect to left and right of the shock-wave.

\subsubsection{Symmetrizing the quark-sub-eikonal corrections}

We observe that the sub-eikonal contribution $\hat{\mathcal{O}}_{1}$ has, like the leading-eikonal term, the operator $\hat{p}$ to its left and to its right, while the sub-eikonal contribution $\hat{\mathcal{O}}_{2}$ has the operator $\hat{p}$ only to its left. We can eliminate the asymmetry between the propagation to the left and to the right of the shock-wave considering the following symmetrization

$$
\left\langle x\left|\frac{i}{\hat{P}+i \epsilon}\right| y\right\rangle=\frac{1}{2}\left\langle x\left|\left[\hat{\not} \frac{i}{p^{2}+i \epsilon}+\frac{i}{\not^{2}+i \epsilon} \hat{P}\right]\right| y\right\rangle .
$$

We have to consider eq. (4.10) and eq. (4.11) with $\not \hat{p} p_{2} \hat{p}$ to the right and the final result for the quark propagator with sub-eikonal corrections can be written in terms of operators $\hat{\mathcal{O}}_{1}$ and $\hat{\mathcal{O}}_{2}$ as

$$
\begin{aligned}
\left\langle x\left|\frac{i}{\hat{p}+i \epsilon}\right| y\right\rangle= & {\left[\int_{0}^{+\infty} \frac{d \alpha}{2 \alpha} \theta\left(x_{*}-y_{*}\right)-\int_{-\infty}^{0} \frac{d \alpha}{2 \alpha} \theta\left(y_{*}-x_{*}\right)\right] e^{-i \alpha\left(x_{\bullet}-y_{\bullet}\right)} \frac{1}{\alpha s} } \\
& \times\left\langle x_{\perp}\right| e^{-i \frac{\hat{p}_{\perp}^{2}}{\alpha s} x_{*}}\left\{\hat{\not p} p_{2}\left[x_{*}, y_{*}\right] \hat{p}+\hat{\not p} p_{2} \hat{\mathcal{O}}_{1}\left(x_{*}, y_{*} ; p_{\perp}\right) \hat{\not p}\right. \\
& \left.+\hat{\not p} p_{2} \frac{1}{2} \hat{\mathcal{O}}_{2}\left(x_{*}, y_{*} ; p_{\perp}\right)-\frac{1}{2} \hat{\mathcal{O}}_{2}\left(x_{*}, y_{*} ; p_{\perp}\right) p_{2} \hat{\not p}\right\} e^{i \frac{\hat{p}_{\perp}^{2}}{\alpha s} y_{*}}\left|y_{\perp}\right\rangle+O\left(\lambda^{-2}\right) .
\end{aligned}
$$




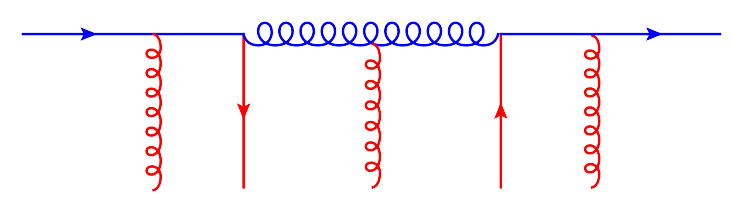

Figure 2. Typical diagram for quark propagator in the background of quark fields. We indicate in blue the quantum field while in red the background one.

The operators $\hat{\mathcal{O}}_{1}, \hat{\mathcal{O}}_{2}$ are the sub-eikonal corrections to the quark propagator that measure the deviation from the straight-line due to the finite width of the shock-wave.

\subsection{Quark propagator in the background of quark and anti-quark fields}

Up to this point we considered only the background gluon field. In this section we consider the propagation of a quark in an external field made of quarks and anti-quarks as well (see figure 2). Our starting point is

$$
\langle\psi(x) \bar{\psi}(y)\rangle_{\psi, \bar{\psi}}=g^{2} \int d^{4} z d^{4} z^{\prime}\left\langle x\left|\frac{1}{\not P+i \epsilon}\right| z\right\rangle \gamma^{\mu} t^{a} \psi(z) \mathcal{G}_{\mu \nu}^{a b}\left(z, z^{\prime}\right) \bar{\psi}\left(z^{\prime}\right) t^{b} \gamma^{\nu}\left\langle z^{\prime}\left|\frac{1}{\not P+i \epsilon}\right| y\right\rangle .
$$

Propagator (4.20) is made of three terms: two quark propagators in the background of gluon field and $\mathcal{G}_{\mu \nu}^{a b}$ the gluon propagator in the background of gluon field. Moreover, at point $z$ and $z^{\prime}$ we have the insertion of the background quark fields. The leading eikonal contribution of propagator (4.20) is

$$
\begin{aligned}
& \langle\psi(x) \bar{\psi}(y)\rangle_{\psi, \bar{\psi}}=g^{2} \int_{y_{*}}^{x_{*}} d z_{*} \int_{y_{*}}^{z_{*}} d z_{*}^{\prime}\left[\int_{0}^{+\infty} \frac{d \alpha}{2 \alpha} \theta\left(x_{*}-y_{*}\right)-\int_{-\infty}^{0} \frac{d \alpha}{2 \alpha} \theta\left(y_{*}-x_{*}\right)\right] e^{-i \alpha\left(x_{\bullet}-y_{\bullet}\right)} \\
& \times \frac{1}{\alpha^{4} s^{4}}\left\langle x_{\perp}\right| e^{-i \frac{\hat{p}_{\perp}^{2}}{\alpha s} x_{*}} \not p p_{2}\left[\not p\left[x_{*}, z_{*}\right] \gamma^{\mu} t^{a} \psi\left(z_{*}\right) \mathcal{G}_{\mu \nu}^{a b}\left(z_{*}, z_{*}^{\prime}\right) \bar{\psi}\left(z_{*}^{\prime}\right) t^{b} \gamma^{\nu}\left[z_{*}^{\prime}, y_{*}\right] p\right. \\
& +g^{2} \frac{2}{s} \gamma^{i} \int_{z_{*}}^{x_{*}} d \omega_{*}\left[x_{*}, \omega_{*}\right] F_{\bullet}\left[\omega_{*}, z_{*}\right] \gamma^{\mu} t^{a} \psi\left(z_{*}\right) \mathcal{G}_{\mu \nu}^{a b}\left(z_{*}, z_{*}^{\prime}\right) \\
& \times \bar{\psi}\left(z_{*}^{\prime}\right) t^{b} \gamma^{\nu} \frac{2}{s} \gamma^{j} \int_{y_{*}}^{z_{*}^{\prime}} d \omega_{*}^{\prime}\left[z_{*}^{\prime}, \omega_{*}^{\prime}\right] F_{\bullet j}\left[\omega_{*}^{\prime}, y_{*}\right] \\
& -g \frac{2}{s} \gamma^{i} \int_{z_{*}}^{x_{*}} d \omega_{*}\left[x_{*}, \omega_{*}\right] F_{\bullet i}\left[\omega_{*}, z_{*}\right] \gamma^{\mu} t^{a} \psi\left(z_{*}\right) \mathcal{G}_{\mu \nu}^{a b}\left(z_{*}, z_{*}^{\prime}\right) \bar{\psi}\left(z_{*}^{\prime}\right) t^{b} \gamma^{\nu}\left[z_{*}^{\prime}, y_{*}\right] p \\
& \left.-\not p\left[x_{*}, z_{*}\right] \gamma^{\mu} t^{a} \psi\left(z_{*}\right) \mathcal{G}_{\mu \nu}^{a b}\left(z_{*}, z_{*}^{\prime}\right) \bar{\psi}\left(z_{*}^{\prime}\right) t^{b} \gamma^{\nu} g \frac{2}{s} \gamma^{i} \int_{y_{*}}^{z_{*}^{\prime}} d \omega_{*}\left[z_{*}^{\prime}, \omega_{*}^{\prime}\right] F_{\bullet}\left[\omega_{*}^{\prime}, y_{*}\right]\right] \\
& \times \not p_{2} \not p e^{i \frac{\hat{p}_{\perp}^{2}}{\alpha s} y_{*}}\left|y_{\perp}\right\rangle+O\left(\lambda^{-2}\right),
\end{aligned}
$$

where $\mathcal{G}_{\mu \nu}^{a b}$ can either be the eikonal term of the gluon propagator in the backgroundFeynman gauge eq. (F.28) or the eikonal term of the light-cone gauge, $p_{2 \mu} A^{\mu}=0$, propagator eq. (5.17). To obtain eq. (4.21) we have used $e^{-i \frac{\hat{p}_{\perp}^{2}}{\alpha s} z_{*}} \psi e^{-i \frac{\hat{p}_{\perp}^{2}}{\alpha s} z_{*}}=\psi+O\left(\lambda^{-1}\right)$ and similarly for $\bar{\psi}$. 
We can simplify further eq. (4.21). Indeed, $D^{\mu} F_{\mu \nu}^{a}=-g \bar{\psi} t^{a} \gamma_{\nu} \psi$ so we can use the following scaling under the boost

$$
\bar{\psi} t^{a} \not p_{1} \psi \rightarrow \lambda \bar{\psi} t^{a} \not p_{1} \psi, \quad \bar{\psi} t^{a} \gamma_{\nu}^{\perp} \psi \rightarrow \bar{\psi} t^{a} \gamma_{\nu}^{\perp} \psi, \quad \bar{\psi} t^{a} \not p_{2} \psi \rightarrow \lambda^{-1} \bar{\psi} t^{a} \not p_{2} \psi
$$

With this power counting in mind, the final result for the quark propagator in the background of gluon field is

$$
\begin{aligned}
\langle\psi(x) \bar{\psi}(y)\rangle_{\psi, \bar{\psi}}= & {\left[\int_{0}^{+\infty} \frac{d \alpha}{2 \alpha} \theta\left(x_{*}-y_{*}\right)-\int_{-\infty}^{0} \frac{d \alpha}{2 \alpha} \theta\left(y_{*}-x_{*}\right)\right] e^{-i \alpha\left(x_{\bullet}-y_{\bullet}\right)} } \\
& \times \frac{g^{2}}{4} \int_{y_{*}}^{x_{*}} d \frac{2}{s} z_{*} \int_{y_{*}}^{z_{*}} d \frac{2}{s} z_{*}^{\prime}\left\langle x_{\perp}\right| e^{-i \frac{\hat{p}_{\perp}^{2}}{\alpha s} x_{*}} \\
& \times\left[x_{*}, z_{*}\right] \not p_{1} \gamma_{\perp}^{\mu} t^{a} \psi\left(z_{*}\right)\left[z_{*}, z_{*}^{\prime}\right]^{a b} \bar{\psi}\left(z_{*}^{\prime}\right) t^{b} \gamma_{\mu}^{\perp} \not p_{1}\left[z_{*}^{\prime}, y_{*}\right] e^{i \frac{\hat{p}_{\perp}^{2}}{\alpha s} y_{*}}\left|y_{\perp}\right\rangle+O\left(\lambda^{-2}\right) .
\end{aligned}
$$

Notice that, the result (4.23) is independent of the gauge used for the gluon propagator.

\section{Sub-eikonal corrections to the gluon propagator}

In this section we calculate the sub-eikonal corrections to the gluon propagator in the in the Light-Cone gauge $p_{2 \mu} A^{\mu}=A_{*}=0$ in the shock-wave limit. In the appendix F.1 we will calculate the gluon propagator in the background-Feynman gauge.

These corrections have been calculated in refs. [26, 27] for an external field $A^{\mu}=$ $\left(A_{\bullet}, 0,0\right)$. We will consider, instead, an external filed with transverse components different than zero, namely, $A^{\mu}=\left(A_{\bullet}, 0, A_{\perp}\right)$.

\subsection{Gluon propagator in the light-cone gauge}

We start considering the gluon propagator in the background of gluon field in functional form

$$
\begin{aligned}
\left\langle A_{\mu}^{a}(x) A_{\nu}^{b}(y)\right\rangle= & \lim _{w \rightarrow 0} N^{-1} \int D A A_{\mu}^{q a}(x) A_{\nu}^{q b}(y) \\
& \times e^{i \int d z \operatorname{Tr}\left\{A_{\alpha}^{q}(z)\left(D^{2} g^{\alpha \beta}-D^{\alpha} D^{\beta}-2 i F_{c l}^{\alpha \beta}-\frac{1}{w} p_{2}^{\alpha} p_{2}^{\beta}\right) A_{\beta}^{q}(z)\right\}},
\end{aligned}
$$

where $D_{\mu}=\partial_{\mu}-i g A_{\mu}^{c l}$. We omit the superscript "cl" from the external field from now on. The issue of fixing properly the sub-gauge conditions will not be discussed here. For this, we refer the reader to ref. [39].

In Schwinger notation the gluon propagator (5.1) can be written as

$$
i\left\langle A_{\mu}^{a}(x) A_{\nu}^{b}(y)\right\rangle \equiv i G_{\mu \nu}^{a b}(x, y)=\left\langle x\left|\frac{1}{\square^{\mu \nu}-P^{\mu} P^{\nu}+\frac{1}{w} p_{2}^{\mu} p_{2}^{\nu}}\right| y\right\rangle^{a b},
$$


where we defined $\square^{\mu \nu}=P^{2} g^{\mu \nu}+2 i g F^{\mu \nu}$. It easy to show that (5.2) satisfies a recursion formula (we omit the symbol` on top of operators, from now on)

$$
\begin{aligned}
\frac{1}{\square^{\mu \nu}-P^{\mu} P^{\nu}+\frac{p_{2}^{\mu} p_{2}^{\nu}}{\omega}}= & \left(\delta_{\mu}^{\xi}-P_{\mu} \frac{p_{2}^{\xi}}{P_{*}}\right) \frac{1}{\square \xi \eta}\left(\delta_{\nu}^{\eta}-\frac{p_{2}^{\eta}}{P_{*}} P_{\nu}\right)+P_{\mu} \frac{\omega}{P_{*}^{2}} P_{\nu} \\
& -\frac{g}{\square^{\mu \alpha}-P^{\mu} P^{\alpha}+\frac{p_{2}^{\mu} p_{2}^{\alpha}}{\omega}}\left(D_{\lambda} F^{\lambda \alpha} \frac{p_{2}^{\beta}}{P_{*}}-P^{\alpha} \frac{1}{P^{2}} D_{\lambda} F^{\lambda \beta}\right) \frac{1}{\square^{\beta \eta}}\left(\delta_{\nu}^{\eta}-\frac{p_{2}^{\eta}}{P_{*}} P_{\nu}\right) \\
& +\frac{g}{\square \mu \alpha-P^{\mu} P^{\alpha}+\frac{p_{2}^{\mu} p_{2}^{\alpha}}{\omega}} D_{\lambda} F^{\lambda \alpha} \frac{\omega}{P_{*}^{2}} P_{\nu} .
\end{aligned}
$$

We need only the terms which are linear and square in the source $D^{\mu} F_{\mu \nu}^{a}(x)=-g \bar{\psi}(x) \gamma_{\nu} t^{a} \psi(x)$ so, from (5.3) we get (now we can also set $w \rightarrow 0$ )

$$
\begin{aligned}
\frac{1}{\square^{\mu \nu}-P^{\mu} P^{\nu}+\frac{p_{2}^{\mu} p_{2}^{\nu}}{\omega}}= & \left(\delta_{\mu}^{\xi}-P_{\mu} \frac{p_{2}^{\xi}}{P_{*}}\right) \frac{1}{\square \xi \eta}\left(\delta_{\nu}^{\eta}-\frac{p_{2}^{\eta}}{P_{*}} P_{\nu}\right) \\
& -\left(\delta_{\mu}^{\xi}-P_{\mu} \frac{p_{2}^{\xi}}{P_{*}}\right) \frac{1}{\square \xi \eta}\left(D_{\lambda} F^{\lambda \eta} \frac{p_{2}^{\beta}}{P_{*}}+\frac{p_{2}^{\eta}}{P_{*}} D_{\lambda} F^{\lambda \beta}\right. \\
& \left.-\frac{p_{2}^{\eta}}{P_{*}} P_{\alpha} D_{\lambda} F^{\lambda \alpha} \frac{p_{2}^{\beta}}{P_{*}}-P^{\eta} \frac{1}{P^{2}} D_{\lambda} F^{\lambda \beta}\right) \frac{1}{\square^{\beta \sigma}}\left(\delta_{\nu}^{\sigma}-\frac{p_{2}^{\sigma}}{P_{*}} P_{\nu}\right) \\
& -\left(\delta_{\mu}^{\xi}-P_{\mu} \frac{p_{2}^{\xi}}{P_{*}}\right) \frac{1}{\square \xi \eta}\left(D_{\lambda} F^{\lambda \eta} \frac{p_{2}^{\beta}}{P_{*}}+\frac{p_{2}^{\eta}}{P_{*}} D_{\lambda} F^{\lambda \beta}\right. \\
& \left.-\frac{p_{2}^{\eta}}{P_{*}} P_{\alpha} D_{\lambda} F^{\lambda \alpha} \frac{p_{2}^{\beta}}{P_{*}}-P^{\eta} \frac{1}{P^{2}} D_{\lambda} F^{\lambda \beta}\right) \frac{1}{\square^{\beta \sigma}} \\
& \times\left(D_{\lambda^{\prime}} F^{\lambda^{\prime} \sigma} \frac{p_{2}^{\beta^{\prime}}}{P_{*}}+\frac{p_{2}^{\sigma}}{P_{*}} D_{\lambda^{\prime}} F^{\lambda^{\prime} \beta^{\prime}}-\frac{p_{2}^{\sigma}}{P_{*}} P_{\alpha} D_{\lambda^{\prime}} F^{\lambda^{\prime} \alpha} \frac{p_{2}^{\beta^{\prime}}}{P_{*}}-P^{\sigma} \frac{1}{P^{2}} D_{\lambda^{\prime}} F^{\lambda^{\prime} \beta^{\prime}}\right) \\
& \times \frac{1}{\square^{\beta^{\prime} \sigma^{\prime}}}\left(\delta_{\nu}^{\sigma^{\prime}}-\frac{p_{2}^{\sigma^{\prime}}}{P_{*}} P_{\nu}\right) .
\end{aligned}
$$

We are interested in calculating corrections up to $\frac{1}{\lambda}$. It is easy to check that we need to expand $\square_{\mu \nu}$ in terms of $F_{\mu \nu}$ up to $F^{3}$ terms (in subsequent algebra we will omit the $+i \epsilon$ prescription for each of the $\frac{1}{P^{2}}$ factor)

$$
i G_{\mu \nu}^{a b}(x, y)=\left\langle x\left|\left(\delta_{\mu}^{\xi}-P_{\mu} \frac{p_{2}^{\xi}}{P_{*}}\right)\left[\frac{g_{\xi \eta}}{P^{2}}-2 i g \frac{1}{P^{2}} F_{\xi \eta} \frac{1}{P^{2}}+\mathcal{O}_{\xi \eta}\right]\left(\delta_{\nu}^{\eta}-\frac{p_{2}^{\eta}}{P_{*}} P_{\nu}\right)\right| y\right\rangle+O\left(\lambda^{-2}\right),
$$

where we defined the operator $\mathcal{O}_{\mu \nu}$ as

$$
\begin{aligned}
\mathcal{O}_{\mu \nu}= & -4 g^{2} \frac{1}{P^{2}} F_{\mu}^{\xi} \frac{1}{P^{2}} F_{\xi \nu} \frac{1}{P^{2}}+8 i g^{3} \frac{1}{P^{2}} F_{\mu}^{\xi} \frac{1}{P^{2}} F_{\xi \eta} \frac{1}{P^{2}} F_{\nu}^{\eta} \frac{1}{P^{2}} \\
& -g \frac{1}{P^{2}}\left(D^{\alpha} F_{\alpha \mu} \frac{p_{2 \nu}}{p_{*}}+\frac{p_{2 \mu}}{p_{*}} D^{\alpha} F_{\alpha \nu}-\frac{p_{2 \mu}}{p_{*}} P^{\beta} D^{\alpha} F_{\alpha \beta} \frac{p_{2 \nu}}{p_{*}}\right) \frac{1}{P^{2}} \\
& +2 i \frac{1}{P^{2}}\left(\frac{p_{2 \mu}}{P_{*}} D_{\lambda} F^{\lambda \beta} \frac{1}{P^{2}} F_{\beta \nu}+F_{\mu \eta} \frac{1}{P^{2}} D_{\lambda} F^{\lambda \eta} \frac{p_{2 \nu}}{P_{*}}\right) \frac{1}{P^{2}} \\
& +\frac{1}{P^{2}}\left(2 D_{\lambda} F^{\lambda} \frac{1}{P^{4}} D_{\rho} F_{\nu}^{\rho}+\frac{p_{2 \mu}}{P_{*}} D_{\lambda} F^{\lambda \beta} \frac{1}{P^{2}} P_{\beta} \frac{1}{P^{2}} D_{\rho} F_{\nu}^{\rho}\right. \\
& \left.-\frac{p_{2 \mu}}{P_{*}} P_{\alpha} D_{\lambda} F^{\lambda \alpha} \frac{1}{P^{4}} D_{\rho} F^{\rho}{ }_{\nu}\right) \frac{1}{P^{2}} .
\end{aligned}
$$


Keeping only terms up to $\lambda^{-1}$, eq. (5.5) becomes

$$
\begin{aligned}
i G_{\mu \nu}^{a b}(x, y)= & {\left[\frac{g_{\mu \nu}}{P^{2}}-2 i \frac{1}{P^{2}}\left(\frac{2}{s} p_{2 \mu} F_{\bullet \nu}+\frac{2}{s} p_{2 \nu} F_{\mu \bullet}\right) \frac{1}{P^{2}}-\frac{1}{P^{2}} \frac{p_{2 \mu}}{p_{*}} P_{\nu}-P_{\mu} \frac{p_{2 \nu}}{p_{*}} \frac{1}{P^{2}}\right.} \\
& \left.+\frac{16 p_{2 \mu} p_{2 \nu}}{s^{2}} \frac{1}{P^{2}} F^{i} \cdot \frac{1}{P^{2}} F_{i \bullet} \frac{1}{P^{2}}-4 \frac{p_{2 \mu} p_{2 \nu}}{\alpha s^{2}} \frac{1}{P^{2}} D^{i} F_{i \bullet} \frac{1}{P^{2}}\right] \\
& -\frac{32 i p_{2 \mu} p_{2 \nu}}{s^{2}} \frac{1}{P^{2}} F^{i} \bullet \frac{1}{P^{2}} F_{i j} \frac{1}{P^{2}} F^{j} \bullet \frac{1}{P^{2}}-2 i \frac{1}{P^{2}} g_{\alpha \mu}^{\perp} g_{\beta \nu}^{\perp} F^{\alpha \beta} \frac{1}{P^{2}} \\
& +\frac{8 p_{2 \mu} g_{\alpha \nu}^{\perp}}{s} \frac{1}{P^{2}} F^{i} \bullet \frac{1}{P^{2}} F_{i}^{\alpha} \frac{1}{P^{2}}+\frac{8 g_{\alpha \mu}^{\perp} p_{2 \nu}}{s} \frac{1}{P^{2}} F^{i \alpha} \frac{1}{P^{2}} F_{i} \bullet \frac{1}{P^{2}} \\
& -\frac{1}{P^{2}} D^{i} F_{i}^{\alpha} g_{\alpha \mu}^{\perp} \frac{p_{2 \nu}}{p_{*}} \frac{1}{P^{2}}-\frac{1}{P^{2}} \frac{p_{2 \mu}}{p_{*}} D^{i} F_{i}{ }^{\alpha} g_{\alpha \nu}^{\perp} \frac{1}{P^{2}}+\frac{1}{P^{2}} \frac{p_{2 \mu}}{p_{*}} P^{i} D^{j} F_{j i} \frac{p_{2 \nu}}{p_{*}} \frac{1}{P^{2}} \\
& +\frac{4 i p_{2 \mu} p_{2 \nu}}{s P_{*}} \frac{1}{P^{2}}\left(D_{i} F^{i j} \frac{1}{P^{2}} F_{j \bullet}-F_{j \bullet} \frac{1}{P^{2}} D_{i} F^{i j}\right) \frac{1}{P^{2}} \\
& +\frac{8 p_{2 \mu} p_{2 \nu}}{s^{2}} \frac{1}{P^{2}} D_{i} F^{i} \bullet \frac{1}{P^{4}} D_{j} F^{j} \cdot \frac{1}{P^{2}} .
\end{aligned}
$$

Note that the terms in square bracket contain both eikonal and sub-eikonal terms, while the terms outside the square bracket are only sub-eikonal terms. After a bit of algebra we can rewrite (5.7) as

$$
\begin{aligned}
i G_{\mu \nu}^{a b}(x, y)= & \langle x|\left\{\left(\delta_{\mu}^{\xi}-\frac{p_{2 \mu}}{p_{*}} P^{\xi}\right) \frac{g_{\xi \eta}}{P^{2}}\left(\delta_{\nu}^{\eta}-P^{\eta} \frac{p_{2 \nu}}{p_{*}}\right)-\frac{p_{2 \mu} p_{2 \nu}}{p_{*}^{2}}\right. \\
& +\left[\frac{4 g^{2} p_{2 \mu} p_{2 \nu}}{s p_{*}} \frac{1}{P^{2}} F^{i} \bullet \frac{1}{P^{2}}\left\{P^{j}, F_{j i}\right\} \frac{1}{P^{2}}+\frac{4 g^{2} p_{2 \mu} p_{2 \nu}}{s p_{*}} \frac{1}{P^{2}}\left\{P^{j}, F_{j i}\right\} \frac{1}{P^{2}} F^{i} \bullet \frac{1}{P^{2}}\right. \\
& +\frac{2 g p_{2 \mu} p_{2 \nu}}{p_{*}^{2}} \frac{1}{P^{2}} P_{i} D_{j} F^{j i} \frac{1}{P^{2}}+\frac{g^{2} p_{2 \mu} p_{2 \nu}}{p_{*}^{2}} \frac{1}{P^{2}} F_{i j} F^{i j} \frac{1}{P^{2}} \\
& -\frac{32 i g^{3} p_{2 \mu} p_{2 \nu}}{s^{2}} \frac{1}{P^{2}} F_{i \bullet} \bullet \frac{1}{P^{2}} F_{i j} \frac{1}{P^{2}} F^{j} \bullet \frac{1}{P^{2}}-2 g i \frac{1}{P^{2}} g_{\alpha \mu}^{\perp} g_{\beta \nu}^{\perp} F^{\alpha \beta} \frac{1}{P^{2}} \\
& +\frac{8 g^{2} p_{2 \mu} g_{\alpha \nu}^{\perp}}{s} \frac{1}{P^{2}} F^{i} \bullet \frac{1}{P^{2}} F_{i}^{\alpha} \frac{1}{P^{2}}+\frac{8 g^{2} g_{\alpha \mu}^{\perp} p_{2 \nu}}{s} \frac{1}{P^{2}} F^{i \alpha} \frac{1}{P^{2}} F_{i} \bullet \frac{1}{P^{2}} \\
& -\frac{1}{P^{2}} g D^{i} F_{i}^{\alpha} g_{\alpha \mu}^{\perp} \frac{p_{2 \nu}}{p_{*}} \frac{1}{P^{2}}-\frac{1}{P^{2}} \frac{p_{2 \mu}}{p_{*}} g D^{i} F_{i}^{\alpha} g_{\alpha \nu}^{\perp} \frac{1}{P^{2}} \\
& +\frac{4 i p_{2 \mu} p_{2 \nu}}{s P_{*}} \frac{1}{P^{2}}\left(D_{i} F^{i j} \frac{1}{P^{2}} F_{j \bullet}-F_{j \bullet} \frac{1}{P^{2}} D_{i} F^{i j}\right) \frac{1}{P^{2}} \\
& \left.\left.+\frac{8 p_{2 \mu} p_{2 \nu}}{s^{2}} \frac{1}{P^{2}} D_{i} F^{i} \bullet \frac{1}{P^{4}} D_{j} F^{j} \bullet \frac{1}{P^{2}}\right]\right\}|y\rangle .
\end{aligned}
$$

In eq. (5.8) we need to make expansion of the operator $P^{2}$. We have to use the leadingeikonal scalar propagator (in the adjoint representation) for the terms that are already sub-eikonal i.e. all terms in the square bracket, while we need to use the scalar propagator with sub-eikonal corrections, eq. (3.7), for the first term right after equal sign in eq. (5.8). Since we are considering the shock-wave case we can disregard the terms with fields at the edges of the gauge links (i.e. at points $x_{*}$ and $y_{*}$ ) in the scalar propagator (3.7). Taking 
all this into account, eq. (5.8) becomes

$$
\begin{aligned}
\left\langle A_{\mu}^{a}(x) A_{\nu}^{b}(y)\right\rangle_{A}= & {\left[-\int_{0}^{+\infty} \frac{d \alpha}{2 \alpha} \theta\left(x_{*}-y_{*}\right)+\int_{-\infty}^{0} \frac{d \alpha}{2 \alpha} \theta\left(y_{*}-x_{*}\right)\right] e^{-i \alpha\left(x_{\bullet}-y_{\bullet}\right)}\left\langle x_{\perp}\right| e^{-i \frac{\hat{p}_{\perp}^{2}}{\alpha s} x_{*}} } \\
& \times\left(\delta_{\mu}^{\xi}-\frac{p_{2 \mu}}{p_{*}} p^{\xi}\right) \mathcal{O}_{\alpha}\left(x_{*}, y_{*}\right)\left(g_{\xi \nu}-p_{\xi} \frac{p_{2 \nu}}{p_{*}}\right) e^{i \frac{\hat{p}_{\perp}^{2}}{\alpha s} y_{*}}\left|y_{\perp}\right\rangle^{a b}+i\left\langle x\left|\frac{p_{2 \mu} p_{2 \nu}}{p_{*}^{2}}\right| y\right\rangle^{a b} \\
& +\left[-\int_{0}^{+\infty} \frac{d \alpha}{2 \alpha} \theta\left(x_{*}-y_{*}\right)+\int_{-\infty}^{0} \frac{d \alpha}{2 \alpha} \theta\left(y_{*}-x_{*}\right)\right] e^{-i \alpha\left(x_{\bullet}-y_{\bullet}\right)}\left\langle x_{\perp}\right| e^{-i \frac{\hat{p}_{\perp}^{2}}{\alpha s} x_{*}} \\
& \times\left[\mathfrak{G}_{1 \mu \nu}^{a b}\left(x_{*}, y_{*} ; p_{\perp}\right)+\mathfrak{G}_{2 \mu \nu}^{a b}\left(x_{*}, y_{*} ; p_{\perp}\right)+\mathfrak{G}_{3 \mu \nu}^{a b}\left(x_{*}, y_{*} ; p_{\perp}\right)+\mathfrak{G}_{4 \mu \nu}^{a b}\left(x_{*}, y_{*} ; p_{\perp}\right)\right] \\
& \times e^{i \frac{\hat{p}_{\perp}^{2}}{\alpha s}} y_{*}\left|y_{\perp}\right\rangle+O\left(\lambda^{-2}\right),
\end{aligned}
$$

where we defined

$$
\begin{aligned}
\mathcal{O}_{\alpha}\left(x_{*}, y_{*}\right) \equiv & {\left[x_{*}, y_{*}\right]+\frac{i g}{2 \alpha} \int_{y_{*}}^{x_{*}} d \frac{2}{s} \omega_{*}\left(\left\{p^{i},\left[x_{*}, \omega_{*}\right] \frac{2}{s} \omega_{*} F_{i \bullet}\left(\omega_{*}\right)\left[\omega_{*}, y_{*}\right]\right\}\right.} \\
& \left.+g \int_{\omega_{*}}^{x_{*}} d \frac{2}{s} \omega_{*}^{\prime} \frac{2}{s}\left(\omega_{*}-\omega_{*}^{\prime}\right)\left[x_{*}, \omega_{*}^{\prime}\right] F^{i} \bullet\left[\omega_{*}^{\prime}, \omega_{*}\right] F_{i \bullet}\left[\omega_{*}, y_{*}\right]\right)
\end{aligned}
$$

and

$$
\begin{aligned}
\mathfrak{G}_{1 \mu \nu}^{a b}\left(x_{*}, y_{*} ; p_{\perp}\right)= & -\frac{g p_{2 \mu} p_{2 \nu}}{s^{2} \alpha^{3}} \int_{y_{*}}^{x_{*}} d \frac{2}{s} \omega_{*}\left[4 p^{i}\left[x_{*}, \omega_{*}\right] F_{i j}\left[\omega_{*}, y_{*}\right] p^{j}\right. \\
& \left.+i g \int_{\omega_{*}^{\prime}}^{x_{*}} d \frac{2}{s} \omega_{*}^{\prime} \frac{2}{s}\left(\omega_{*}^{\prime}-\omega_{*}\right)\left[x_{*}, \omega_{*}^{\prime}\right] i D^{i} F_{i \bullet}\left[\omega_{*}^{\prime}, \omega_{*}\right] i D^{j} F_{j \bullet}\left[\omega_{*}, y_{*}\right]\right]^{a b}, \\
\mathfrak{G}_{2 \mu \nu}^{a b}\left(x_{*}, y_{*} ; p_{\perp}\right)= & -\frac{g}{\alpha} \delta_{\mu}^{i} \delta_{\nu}^{j} \int_{y_{*}}^{x_{*}} d \frac{2}{s} \omega_{*}\left(\left[x_{*}, \omega_{*}\right] F_{i j}\left[\omega_{*}, y_{*}\right]\right)^{a b}, \\
\mathfrak{G}_{3 \mu \nu}^{a b}\left(x_{*}, y_{*} ; p_{\perp}\right)= & \frac{g}{\alpha^{2} s}\left(\delta_{\mu}^{j} p_{2 \nu}+\delta_{\nu}^{j} p_{2 \mu}\right) \int_{y_{*}}^{x_{*}} d \frac{2}{s} \omega_{*}\left(\left[x_{*}, \omega_{*}\right] i D^{i} F_{i j}\left[\omega_{*}, y_{*}\right]\right)^{a b}, \\
\mathfrak{G}_{4 \mu \nu}^{a b}\left(x_{*}, y_{*} ; p_{\perp}\right)= & -\frac{2 g^{2}}{\alpha^{2} s} \int_{y_{*}}^{x_{*}} d \frac{2}{s} \omega_{*} \int_{\omega_{*}}^{x_{*}} d \frac{2}{s} \omega_{*}^{\prime}\left(\delta_{\nu}^{j} p_{2 \mu}\left[x_{*} \omega_{*}^{\prime}\right] F_{\bullet}^{i}\left[\omega_{*}^{\prime}, \omega_{*}\right] F_{i j}\left[\omega_{*}, y_{*}\right]\right. \\
& \left.+\delta_{\mu}^{j} p_{2 \nu}\left[x_{*} \omega_{*}^{\prime}\right] F_{i j}\left[\omega_{*}^{\prime}, \omega_{*}\right] F_{\bullet}^{i}\left[\omega_{*}, y_{*}\right]\right)^{a b} .
\end{aligned}
$$

Equation (5.9) is our final result for the gluon propagator with sub-eikonal corrections in the light-cone gauge. At this point we can send $x_{*} \rightarrow \infty$ and $y_{*} \rightarrow-\infty$ and obtain the gluon propagator in the light-cone gauge with sub-eikonal corrections in the shock-wave case. 

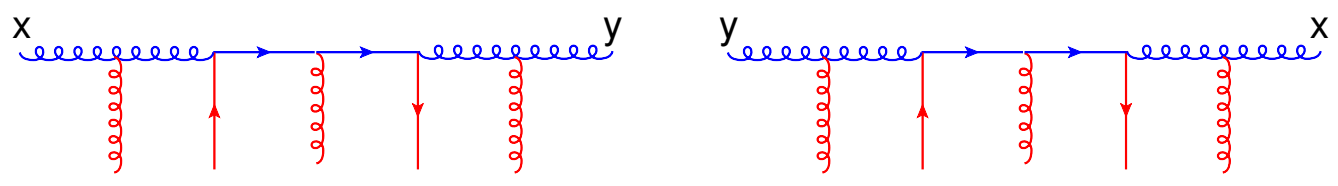

Figure 3. Typical diagram and cross-diagram for gluon propagator in the background of quark fields. As usual, we indicate in blue the quantum field while in red the background one.

\subsection{Gluon propagator in the light-cone gauge in the background of quark and anti-quark fields}

In the previous section we considered the gluon propagator in the axial gauge in the background of gluon fields. We now consider the gluon propagator in the background of quarks and anti-quarks in the axial gauge. In section F.2 we will consider the backgroundFeynman gauge.

The Feynman diagram is given in figure 3

$$
\begin{aligned}
\left\langle A_{\mu}^{a}(x) A_{\nu}^{b}(y)\right\rangle_{\psi, \bar{\psi}}= & -g^{2} \int d^{4} z_{1} d^{4} z_{2}\left\langle A_{\mu}^{a}(x) A_{\rho}^{c}\left(z_{1}\right)\right\rangle\left\langle A_{\lambda}^{d}\left(z_{2}\right) A_{\nu}^{b}(y)\right\rangle \\
& \times\left[\bar{\psi}\left(z_{2}\right) t^{d} \gamma^{\lambda}\left\langle z_{2}\left|\frac{i}{P+i \epsilon}\right| z_{1}\right\rangle \gamma^{\rho} t^{c} \psi\left(z_{1}\right)+\bar{\psi}\left(z_{1}\right) t^{c} \gamma^{\rho}\left\langle z_{1}\left|\frac{i}{\not P+i \epsilon}\right| z_{2}\right\rangle \gamma^{\lambda} t^{d} \psi\left(z_{2}\right)\right] .
\end{aligned}
$$

To disentangle the eikonal terms from the sub-eikonal ones, we will use again the scaling in eq. (4.22). The first thing we observe is that the gluon propagator in the axial gauge in the background of quark and anti-quark fields starts already with sub-eikonal contribution. Therefore, it is sufficient to use the eikonal gluon propagator (5.9) and the eikonal quark propagator (2.9) to obtain

$$
\begin{aligned}
\left\langle A_{\mu}^{a}(\right. & \left.(x) A_{\nu}^{b}(y)\right\rangle_{\psi, \bar{\psi}} \\
= & {\left[-\int_{0}^{+\infty} \frac{d \alpha}{2 \alpha} \theta\left(x_{*}-y_{*}\right)+\int_{-\infty}^{0} \frac{d \alpha}{2 \alpha} \theta\left(y_{*}-x_{*}\right)\right] e^{-i \alpha\left(x_{\bullet}-y_{\bullet}\right)} } \\
& \times g^{2} \int_{y_{*}}^{x_{*}} d \frac{2}{s} z_{1 *} \int_{y_{*}}^{z_{1 *}} d \frac{2}{s} z_{2 *} \frac{1}{4 \alpha}\left[\left\langle x_{\perp}\right| e^{-i \frac{\hat{p}_{\perp}^{2}}{\alpha s} x_{*}}\left(g_{\perp \mu}^{\xi}-\frac{p_{2 \mu}}{p_{*}} p_{\perp}^{\xi}\right)\right. \\
& \times \bar{\psi}\left(z_{1 *}\right) \gamma_{\xi}^{\perp} p_{1}\left[z_{1 *}, x_{*}\right] t^{a}\left[x_{*}, y_{*}\right] t^{b}\left[y_{*}, z_{2 *}\right] \gamma_{\perp}^{\sigma} \psi\left(z_{2 *}\right)\left(g_{\sigma \nu}^{\perp}-p_{\sigma}^{\perp} \frac{p_{2 \nu}}{p_{*}}\right) e^{i \frac{\hat{p}_{\perp}^{2}}{\alpha s} y_{*}}\left|y_{\perp}\right\rangle \\
& +\left\langle y_{\perp}\right| e^{-i \frac{\hat{p}_{\perp}^{2}}{\alpha s} y_{*}}\left(g_{\perp \nu}^{\xi}-\frac{p_{2 \nu}}{p_{*}} p_{\perp}^{\xi}\right) \bar{\psi}\left(z_{2 *}\right) \gamma_{\xi}^{\perp} \not p_{1}\left[z_{2 *}, y_{*}\right] t^{b}\left[y_{*}, x_{*}\right] t^{a}\left[x_{*}, z_{1 *}\right] \gamma_{\perp}^{\sigma} \psi\left(z_{1 *}\right) \\
& \left.\times\left(g_{\sigma \mu}^{\perp}-p_{\sigma}^{\perp} \frac{p_{2 \mu}}{p_{*}}\right) e^{i \frac{\hat{p}_{\perp}^{2}}{\alpha s} x_{*}}\left|x_{\perp}\right\rangle\right]+O\left(\lambda^{-2}\right)
\end{aligned}
$$

where $p_{*}=\frac{s}{2} \alpha$. To arrive at result (5.16) we have used $e^{-i \frac{\hat{p}_{\perp}^{2}}{\alpha s} z_{*}} \psi\left(z_{*}\right) e^{i \frac{\hat{p}_{\perp}^{2}}{\alpha s} z_{*}}=\psi\left(z_{*}\right)+O(\lambda)$ and similarly for $\bar{\psi}$.

Equation (5.16) is the gluon propagator in the background of quark and anti-quark fields. Contrary to the background-Feynman gauge, as we will see in the appendix, in the axial gauge the gluon propagator in the quark anti-quark external fields does not have any eikonal terms but only sub-eikonal ones. 


\subsection{Summing up the gluon and quark anti-quark external fields contributions}

We can now write the final expression for the gluon propagator in the light-cone gauge in the gluon and quark anti-quark external fields up to sub-eikonal corrections as

$$
\begin{aligned}
& \left\langle A_{\mu}^{a}(x) A_{\nu}^{b}(y)\right\rangle=\left[-\int_{0}^{+\infty} \frac{d \alpha}{2 \alpha} \theta\left(x_{*}-y_{*}\right)+\int_{-\infty}^{0} \frac{d \alpha}{2 \alpha} \theta\left(y_{*}-x_{*}\right)\right] e^{-i \alpha\left(x_{\bullet}-y_{\bullet}\right)}\left\langle x_{\perp}\right| e^{-i \frac{\hat{p}_{\perp}^{2}}{\alpha s} x_{*}} \\
& \quad \times\left(g_{\perp \mu}^{\xi}-\frac{p_{2 \mu}}{p_{*}} p_{\perp}^{\xi}\right) \mathcal{O}_{\alpha}\left(x_{*}, y_{*}\right)\left(g_{\xi \nu}^{\perp}-p_{\xi}^{\perp} \frac{p_{2 \nu}}{p_{*}}\right) e^{i \frac{\hat{p}_{\perp}^{2}}{\alpha s} y_{*}}\left|y_{\perp}\right\rangle^{a b}+i\left\langle x\left|\frac{p_{2 \mu} p_{2 \nu}}{p_{*}^{2}}\right| y\right\rangle^{a b} \\
& \quad+\left[-\int_{0}^{+\infty} \frac{d \alpha}{2 \alpha} \theta\left(x_{*}-y_{*}\right)+\int_{-\infty}^{0} \frac{d \alpha}{2 \alpha} \theta\left(y_{*}-x_{*}\right)\right] e^{-i \alpha\left(x_{\bullet}-y_{\bullet}\right)}\left\{\left\langle x_{\perp}\right| e^{-i \frac{\hat{p}_{\perp}^{2}}{\alpha s} x_{*}}\right. \\
& \quad \times\left(\mathfrak{G}_{1 \mu \nu}^{a b}\left(x_{*}, y_{*} ; p_{\perp}\right)+\mathfrak{G}_{2 \mu \nu}^{a b}\left(x_{*}, y_{*} ; p_{\perp}\right)+\mathfrak{G}_{3 \mu \nu}^{a b}\left(x_{*}, y_{*} ; p_{\perp}\right)+\mathfrak{G}_{4 \mu \nu}^{a b}\left(x_{*}, y_{*} ; p_{\perp}\right)\right)\left|y_{\perp}\right\rangle e^{i \frac{\hat{p}_{\perp}^{2}}{\alpha s} y_{*}} \\
& \quad+g^{2} \int_{y_{*}}^{x_{*}} d \frac{2}{s} z_{1 *} \int_{y_{*}}^{z_{1 *}} d \frac{2}{s} z_{2 *} \frac{1}{4 \alpha}\left[\left\langle x_{\perp}\right| e^{-i \frac{\hat{p}_{\perp}^{2}}{\alpha s} x_{*}}\left(g_{\perp \mu}^{\xi}-\frac{p_{2 \mu}}{p_{*}} p_{\perp}^{\xi}\right)\right. \\
& \quad \times \bar{\psi}\left(z_{1 *}\right) \gamma_{\xi}^{\perp} \not p_{1}\left[z_{1 *}, x_{*}\right] t^{a}\left[x_{*}, y_{*}\right] t^{b}\left[y_{*}, z_{2 *}\right] \gamma_{\perp}^{\sigma} \psi\left(z_{2 *}\right)\left(g_{\sigma \nu}^{\perp}-p_{\sigma}^{\perp} \frac{p_{2 \nu}}{p_{*}}\right) e^{i \frac{\hat{p}_{\perp}^{2}}{\alpha s} y_{*}}\left|y_{\perp}\right\rangle \\
& \quad+\left\langle y_{\perp}\right| e^{-i \frac{\hat{p}_{\perp}^{2}}{\alpha s} y_{*}}\left(g_{\perp \nu}^{\xi}-\frac{p_{2 \nu}}{p_{*}} p_{\perp}^{\xi}\right) \bar{\psi}\left(z_{2 *}\right) \gamma_{\xi}^{\perp} p_{1}\left[z_{2 *}, y_{*}\right] t^{b}\left[y_{*}, x_{*}\right] t^{a}\left[x_{*}, z_{1 *}\right] \gamma_{\perp}^{\sigma} \psi\left(z_{1 *}\right) \\
& \left.\quad \times\left(g_{\sigma \mu}^{\perp}-p_{\sigma}^{\perp} \frac{p_{2 \mu}}{p_{*}}\right) e^{i \frac{\hat{p}_{\perp}^{2}}{\alpha s} x_{*}}\left|x_{\perp}\right\rangle\right]+O\left(\lambda^{-2}\right),
\end{aligned}
$$

All terms in curly brackets in eq. (5.17) are the sub-eikonal corrections: they scale as $\lambda^{-1}$ under the longitudinal boost.

\section{Conclusions and outlook}

In the high-energy OPE formalism, in the eikonal approximation, the evolution equations of the relevant operators, the infinite Wilson lines, are the non-linear evolution BK or B-JIMWLK evolution equations. When sub-eikonal terms are included, new operators appear and, consequently, new non-linear evolution equations need to be derived. The new evolution equations will describe, for example, the high-energy dynamics of scattering processes with spin. These sub-eikonal corrections to high-energy OPE are similar to higher-twist corrections to the usual light-ray OPE [40] which are now important part of QCD phenomenology [41-44].

The advantage of the operatorial formalism adopted in this paper is provided by both the gauge invariant representation of the sub-eikonal operators, and by the systematics provided by the OPE formalism. Indeed, given an operatorial definition of the observable, like, for example, the T-product of two electromagnetic currents in DIS, the application of the high-energy OPE will provide a systematic description of the observable in terms of coefficient functions and matrix elements of the relevant operators evaluated in the target state. The relevant operators are provided by the propagation of the projectile-particle in the background of the target-external field like the propagator in eq. (4.19). 
The main results obtained in this paper are the sub-eikonal corrections to the quark propagator given in eq. (4.19), and to the gluon propagator in the light-cone gauge given in eq. (5.9).

The first step we made was the calculation of the scalar propagator and its sub-eikonal corrections given in eq. (3.7). To obtained this first result, we showed that the term $\left\{\hat{P}_{\bullet}, A_{*}\right\}$, which is related to the possibility for the fields to have dependence also on $x_{\bullet}$, is actually a sub-sub-eikonal correction. We proved it in two different ways. We have also shown that the non gauge-invariant terms at the edges of the gauge fields in eq. (3.7), which can be put to zero being pure gauges, actually play an important role: when the scalar propagator is used in intermediate steps, these non gauge-invariant terms may be in the middle of the shock-wave and therefore cannot be put to zero.

In the quark propagator eq. (4.19), the new gauge invariant operators are given in the definition of $\hat{\mathcal{O}}_{1}, \hat{\mathcal{O}}_{2}$ in eqs. (4.13) and (4.14) respectively.

To get the sub-eikonal terms we had to assume that the external field has a finite width. As a consequence one has to consider also the possibility that the scattering particle starts (or ends) its propagation inside the target-filed. To this end, we have derived scalar propagator (E.4) and the quark propagator (E.9) which take into account this possibility.

In section 5.1 we have calculated the sub-eikonal corrections to the gluon propagator in the light-cone gauge. In ref. [27] such correction have been calculated for a background field $A^{\mu}(x)=\left(A_{\bullet}\left(x_{*}, x_{\perp}\right), 0,0\right)$. Here we considered an external field where all the components of the external field are different then zero. The result is given in eq. (5.17); it includes the contribution due to both the gluon and quark anti-quark external fields. The operators $\mathfrak{G}_{1 \mu \nu}^{a b}, \mathfrak{G}_{2 \mu \nu}^{a b}, \mathfrak{G}_{3 \mu \nu}^{a b}$ and $\mathfrak{G}_{4 \mu \nu}^{a b}$, given in equations (5.11), (5.12), (5.13) and (5.14), respectively, are the result of an external gluon field with all filed components different then zero, while eq. (5.16) is the sub-eikonal corrections due to quark anti-quark external fields. In background-Feynman gauge, instead, the gluon propagator is given in eq. (F.28), and the sub-eikonal correctins are all included in the operators $\mathfrak{B}_{1 \mu \nu}^{a b}, \mathfrak{B}_{2 \mu \nu}^{a b}, \mathfrak{B}_{3 \mu \nu}^{a b}, \mathfrak{B}_{4 \mu \nu}^{a b}, \mathfrak{B}_{5 \mu \nu}^{a b}$, $\mathfrak{B}_{6 \mu \nu}^{a b}$ and $\mathfrak{Q}_{1 \mu \nu}^{a b}, \mathfrak{Q}_{2 \mu \nu}^{a b}, \mathfrak{Q}_{3 \mu \nu}^{a b}, \mathfrak{Q}_{4 \mu \nu}^{a b}, \mathfrak{Q}_{5 \mu \nu}^{a b}$ in eqs. (F.4), (F.5), (F.6), (F.7), (F.8), and (F.9) and (F.22), (F.23), (F.24), (F.25), (F.26), respectively.

In refs. $[32,33]$ it was shown that both the gluon and the quark distribution contribute equally to the spin structure function at low- $x_{B}$. This result was obtained within the double-logarithmic approximation (DLA). To study the quark distribution within the Wilson-line formalism, in section 4.2 , we considered also the sub-eikonal corrections due to quark and anti-quark in the target-external field. The quark propagator with such subeikonal corrections is given in eq. (4.23). Not only will this result be relevant for high-energy spin-dynamics but also to obtain sub-eikonal corrections to the BK equation. Although such corrections to the BK equations are energy suppressed, one may obtain for the first time the Regge limit of scattering amplitudes with two-fermions in the t-channel [34] within the Wilson-line formalism.

The applications of the results derived in this paper does not end with high-energy spin-dynamics. The TMD formalism developed so far (for a review see, [45]) does not describe data at sufficiently low- $x_{B}$. If one wants to have at hand a formalism that can be applicable at a wider range of $x_{B}$, one has to consider sub-eikonal corrections as a 
way to connect to lower energies and thus moderate $x_{B}$. In the case of gluon TMDs this connection has been already made in refs. [26, 27]. In the case of quark-TMDs, instead, one my use the results derived in this paper, although here we have not included terms coming from twist expansion since we considered classical fields and quantum fields with comparable transverse momenta. Corrections due to the hierarchy between the transverse momenta of the quantum and classical field is left for future publication.

The author is grateful to I. Balitsky and V.M. Braun for valuable discussions.

\section{A Notation}

In this section we explain some of the notations used through out the paper.

Given two light-cone vectors $p_{1}^{\mu}$ and $p_{2}^{\mu}$, with $p_{1}^{\mu} p_{2 \mu}=\frac{s}{2}$, we can decompose any coordinate as $x^{\mu}=\frac{2}{s} x_{*} p_{1}^{\mu}+\frac{2}{s} x_{\bullet} p_{2}^{\mu}+x_{\perp}^{\mu}$ with $x_{*}=x_{\mu} p_{2}^{\mu}=\sqrt{\frac{s}{2}} x^{+}, x_{\bullet}=x_{\mu} p_{1}^{\mu}=\sqrt{\frac{s}{2}} x^{-}$ and $x^{ \pm}=\frac{x^{0} \pm x^{3}}{\sqrt{2}}$. We use the notation $x_{\perp}^{\mu}=\left(0, x^{1}, x^{2}, 0\right)$ and $x^{i}=\left(x^{1}, x^{2}\right)$ such that $x^{i} x_{i}=x_{\perp}^{\mu} x_{\mu}^{\perp}=-x_{\perp}^{2}$. So, Latin indexes assume values 1,2 , while Greek indexes run from 0 to 3 .

We define the gauge link at fixed transverse position as

$$
\left[u p_{1}, v p_{2}\right]_{z} \equiv\left[u p_{1}+z_{\perp}, v p_{1}+z_{\perp}\right] \equiv \operatorname{Pexp}\left\{i g \int_{v}^{u} d t A_{\bullet}\left(t p_{1}+z_{\perp}\right)\right\} .
$$

The derivative of the gauge link with respect to the transverse position is

$$
\begin{aligned}
\frac{\partial}{\partial z^{i}}\left[u p_{1}, v p_{1}\right]_{z}= & i g A_{i}\left(u p_{1}+z_{\perp}\right)\left[u p_{1}, v p_{1}\right]_{z}-i g\left[u p_{1}, v p_{1}\right]_{z} A_{i}\left(v p_{1}+z_{\perp}\right) \\
& -i g \int_{v}^{u} d s\left[u p_{1}, s p_{1}\right]_{z} F_{\bullet}\left(p_{1} s+z_{\perp}\right)\left[p_{1} s, p_{1} v\right]_{z}
\end{aligned}
$$

with index $i=1,2$. From (A.2) we may formally define the transverse covariant derivative $\mathfrak{D}_{i}$ that acts on a non-local operator as

$$
\begin{aligned}
i \mathfrak{D}_{i}\left[u p_{1}, v p_{1}\right]_{z} & \equiv i \frac{\partial}{\partial z^{i}}\left[u p_{1}, v p_{1}\right]_{z}+g\left[A_{i}\left(z_{\perp}\right),\left[u p_{1}, v p_{1}\right]_{z}\right] \\
& =g \int_{v}^{u} d s\left[u p_{1}, s p_{1}\right]_{z} F_{\bullet}\left(p_{1} s+z_{\perp}\right)\left[p_{1} s, p_{1} v\right]_{z},
\end{aligned}
$$

where we have used the implicit notation $\left[A_{i}\left(z_{\perp}\right),\left[u p_{1}, v p_{1}\right]_{z}\right]=A_{i}\left(z_{\perp}+u p_{1}\right)\left[u p_{1}, v p_{1}\right]_{z}-$ $\left[u p_{1}, v p_{1}\right]_{z} A_{i}\left(z_{\perp}+v p_{1}\right)$.

Given a gauge link $\left[x_{*}, y_{*}\right]_{z} \equiv\left[\frac{2}{s} x_{*} p_{1}+z_{\perp}, \frac{2}{s} y_{*} p_{1}+z_{\perp}\right]$, in Schwinger notation we have

$$
\left\langle x_{\perp}\left|\left[x_{*}, y_{*}\right]\right| y_{\perp}\right\rangle=\left[x_{*}, y_{*}\right]_{x} \delta^{(2)}(x-y) .
$$

The transverse momentum operator $\hat{P}_{i}=\hat{p}_{i}+g \hat{A}_{i}$ acts on the gauge link as

$$
\left\langle x_{\perp}\left|\left[\hat{P}_{i},\left[x_{*}, y_{*}\right]\right]\right| y_{\perp}\right\rangle=\left\langle x_{\perp}\left|i \mathfrak{D}_{i}\left[x_{*}, y_{*}\right]\right| y_{\perp}\right\rangle=\left\langle x_{\perp}\left|g \frac{2}{s} \int_{y_{*}}^{x_{*}} d \omega_{*}\left[x_{*}, \omega_{*}\right] F_{\bullet i}\left[\omega_{*}, y_{*}\right]\right| y_{\perp}\right\rangle
$$

where we used again the short-hand notation $\left[x_{*}, \omega_{*}\right] F_{i \bullet}\left[\omega_{*}, y_{*}\right]=\left[x_{*}, \omega_{*}\right] F_{i \bullet}\left(\omega_{*}\right)\left[\omega_{*}, y_{*}\right]$. The point to make regarding (A.5) is that the covariant derivative $i \mathfrak{D}_{i}$ acts on the gauge link 
even though the transverse coordinate has not been specified yet and, as matter of fact, it does not have to in order to know how it acts on the gauge link. Therefore, through out the paper we will make quite a bit of algebra involving the gauge link, the momentum operator $P_{i}$ and the covariant derivative $i \mathfrak{D}_{i}$ without specifying the bra $\left\langle x_{\perp}\right|$ and the ket $\left|y_{\perp}\right\rangle$.

At this point, one is tempted to identify the covariant derivative $i \mathfrak{D}_{i}$ as the usual covariant derivative which acts on a local operator. This identification would not be correct. Indeed, one can easily check that

$$
\left[i \mathfrak{D}_{i}, i \mathfrak{D}_{j}\right]\left[x_{*}, y_{*}\right]=i g F_{i j}\left[x_{*}, y_{*}\right]-i g\left[x_{*}, y_{*}\right] F_{i j} .
$$

To arrive at (A.6) we have implemented the definition of $i \mathfrak{D}_{i}$ given in (A.3).

Another identity involving $\mathfrak{D}_{i}$ that we will often use is

$$
\begin{aligned}
i \mathfrak{D}_{j}\left(\left[x_{*}, \omega_{*}\right] F_{\bullet}\left[\omega_{*}, y_{*}\right]\right)= & \left(i \mathfrak{D}_{j}\left[x_{*}, \omega_{*}\right]\right) F_{\bullet}\left[\omega_{*}, y_{*}\right]+\left[x_{*}, \omega_{*}\right] F_{\bullet i}\left(i \mathfrak{D}_{j}\left[\omega_{*}, y_{*}\right]\right) \\
& +\left[x_{*}, \omega_{*}\right]\left(i D_{j} F_{\bullet i}\right)\left[\omega_{*}, y_{*}\right] \\
= & g \frac{2}{s} \int_{\omega_{*}}^{x_{*}} d z_{*}\left[x_{*}, z_{*}\right] F_{\bullet j}\left[z_{*}, \omega_{*}\right] F_{\bullet}\left[\omega_{*}, y_{*}\right] \\
& +g \frac{2}{s} \int_{y_{*}}^{\omega_{*}} d z_{*}\left[x_{*}, \omega_{*}\right] F_{\bullet i}\left[\omega_{*}, z_{*}\right] F_{\bullet j}\left[z_{*}, y_{*}\right] \\
& +\left[x_{*}, \omega_{*}\right]\left(i D_{j} F_{\bullet i}\right)\left[\omega_{*}, y_{*}\right] .
\end{aligned}
$$

Notice that in eq. (A.7) derivative $\mathfrak{D}_{i}$ acts on the gauge link, while the usual covariant derivative $D_{j}$ acts on the local field operator $F_{\bullet} i$ with respect to the transverse coordinate that will be specified in a second step according to Schwinger notation (A.4). So, as we can see from the first line after the equal sign in eq. (A.7), $\mathfrak{D}_{i}$ follows the usual Leibnitz rule for derivative of product of functions with the exception that when $\mathfrak{D}_{i}$ acts on a gauge link it acts as in eq. (A.3), while when it acts on a local filed operator it becomes the usual covariant derivative. It is now easy to show that

$$
\begin{aligned}
\mathfrak{D}_{\perp}^{2}\left[x_{*}, y_{*}\right]= & 2 g^{2} \int_{y_{*}}^{x_{*}} d \frac{2}{s} \omega_{*} \int_{\omega_{*}}^{x_{*}} d \frac{2}{s} \omega_{*}^{\prime}\left[x_{*}, \omega_{*}^{\prime}\right] F_{i \bullet}\left[\omega_{*}^{\prime}, \omega_{*}\right] F_{\bullet}^{i}\left[\omega_{*}, y_{*}\right] \\
& +g \int_{y_{*}}^{x_{*}} d \frac{2}{s} \omega_{*}\left[x_{*}, \omega_{*}\right]\left(i D^{i} F_{\bullet}\right)\left[\omega_{*}, y_{*}\right]
\end{aligned}
$$

where $\mathfrak{D}_{\perp}^{2}=-\mathfrak{D}^{i} \mathfrak{D}_{i}$.

\section{B An alternative type of expansion for the quark propagator}

The procedure we adopted in section 2.2 to obtain the quark propagator in the eikonal approximation is certainly not the only one. In this section we consider the following type of expansion

$$
\begin{aligned}
\left\langle x\left|\frac{1}{p+i \epsilon}\right| y\right\rangle= & \left\langle x\left|\frac{1}{\not p+i \epsilon}\right| y\right\rangle-\left\langle x\left|\frac{1}{\not p+i \epsilon} g A \frac{1}{\not p+i \epsilon}\right| y\right\rangle \\
& +\left\langle x\left|\frac{1}{\not p+i \epsilon} g A \frac{1}{\not p+i \epsilon} g A \frac{1}{\not p+i \epsilon}\right| y\right\rangle+\ldots,
\end{aligned}
$$




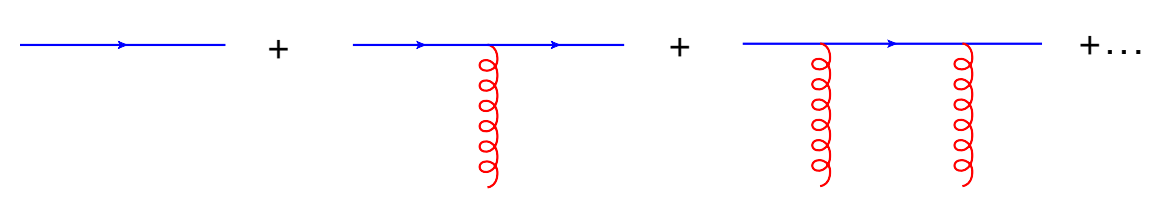

Figure 4. Diagrammatic expansion of the quark propagator in the external field. Quantum fields are in blue, while classical fields are in red as usual.

which is diagrammatically shown in figure 4 . Now we are interested only in the eikonal contribution, so we can use, in Schwinger notation, $\langle x|A| y\rangle=\frac{2}{s} \not p_{2} A_{\bullet}\left(x_{*}, x_{\perp}\right) \delta^{(4)}(x-y)$ and eq. (B.1) becomes

$$
\begin{aligned}
& \left\langle x\left|\frac{1}{\not P+i \epsilon}\right| y\right\rangle=\frac{\not x-y}{2 \pi^{2}\left[(x-y)^{2}-i \epsilon\right]^{2}}-\frac{i}{s} \int_{y_{*}}^{x_{*}} d z_{*}\left[\int_{0}^{+\infty} \frac{d \alpha}{2 \alpha^{2}} \theta\left(x_{*}-z_{*}\right) \theta\left(z_{*}-y_{*}\right)\right. \\
& \left.-\int_{-\infty}^{0} \frac{d \alpha}{2 \alpha^{2}} \theta\left(y_{*}-z_{*}\right) \theta\left(z_{*}-x_{*}\right)\right] e^{-i \alpha\left(x_{\bullet}-y_{\bullet}\right)} \\
& \times\left\langle x_{\perp}\left|\hat{p} e^{-i \frac{\hat{p}_{\perp}^{2}}{\alpha s}\left(x_{*}-z_{*}\right)} i g \frac{2}{s} \not p_{2} \hat{A} \bullet\left(z_{*}\right) e^{-i \frac{\hat{p}_{\perp}^{2}}{\alpha s}\left(z_{*}-y_{*}\right)} \hat{\not p}\right| y_{\perp}\right\rangle \\
& -\frac{i}{s} \int_{y_{*}}^{x_{*}} d z_{*} \int_{y_{*}}^{z_{*}} d z_{*}^{\prime}\left[\int_{0}^{+\infty} \frac{d \alpha}{2 \alpha^{2}} \theta\left(x_{*}-z_{*}\right) \theta\left(z_{*}-z_{*}^{\prime}\right) \theta\left(z_{*}^{\prime}-y_{*}\right)\right. \\
& \left.-\int_{-\infty}^{0} \frac{d \alpha}{2 \alpha^{2}} \theta\left(y_{*}-z_{*}^{\prime}\right) \theta\left(z_{*}^{\prime}-z_{*}\right) \theta\left(z_{*}-x_{*}\right)\right] e^{-i \alpha\left(x_{\bullet}-y_{\bullet}\right)} \\
& \times\left\langle x_{\perp}\left|\hat{p} e^{-i \frac{\hat{p}_{\perp}^{2}}{\alpha s}\left(x_{*}-z_{*}\right)} i g \frac{2}{s} \not p_{2} \hat{A} \bullet\left(z_{*}\right) e^{-i \frac{\hat{p}_{\perp}^{2}}{\alpha s}\left(z_{*}-z_{*}^{\prime}\right)} i g \frac{2}{s} \hat{A} \bullet\left(z_{*}^{\prime}\right) e^{-i \frac{\hat{p}_{\perp}^{2}}{\alpha s}\left(z_{*}^{\prime}-y_{*}\right)} \hat{p}\right| y_{\perp}\right\rangle .
\end{aligned}
$$

We can rewrite expansion (B.2) as a path ordered exponential

$$
\begin{aligned}
\left\langle x\left|\frac{i}{\hat{p}+i \epsilon}\right| y\right\rangle= & \frac{1}{s}\left[\int_{0}^{+\infty} \frac{d \alpha}{2 \alpha^{2}} \theta\left(x_{*}-y_{*}\right)-\int_{-\infty}^{0} \frac{d \alpha}{2 \alpha^{2}} \theta\left(y_{*}-x_{*}\right)\right] e^{-i \alpha\left(x_{\bullet}-y_{\bullet}\right)}\left\langle x_{\perp}\right| \hat{p} e^{-i \frac{\hat{p}_{\perp}^{2}}{\alpha s} x_{*}} \\
& \times p_{2} \operatorname{Pexp}\left\{i g \int_{y_{*}}^{x_{*}} d \frac{2}{s} z_{*} e^{-i \frac{\hat{p}_{\perp}^{2}}{\alpha s} z_{*}} \hat{A} \bullet\left(z_{*}\right) e^{i \frac{\hat{p}_{\perp}^{2}}{\alpha s} z_{*}}\right\} e^{i \frac{\hat{p}_{\perp}^{2}}{\alpha s} y_{*}} \hat{p}\left|y_{\perp}\right\rangle
\end{aligned}
$$

We may perform a further approximation due to the infinite boost (we are interest only in the eikonal contribution)

$$
e^{-i \frac{\hat{p}_{\perp}^{2}}{\alpha s} z_{*}} \hat{A}_{\bullet}\left(z_{*}\right) e^{i \frac{\hat{p}_{\perp}^{2}}{\alpha s} z_{*}}=\hat{A}_{\bullet}\left(z_{*}\right)+O\left(\lambda^{0}\right)
$$

where $\lambda$ is the large parameter of the Lorentz boost. With this approximation we arrive at

$$
\begin{aligned}
\left\langle x\left|\frac{i}{\hat{p}+i \epsilon}\right| y\right\rangle= & \frac{1}{s}\left[\int_{0}^{+\infty} \frac{d \alpha}{2 \alpha^{2}} \theta\left(x_{*}-y_{*}\right)-\int_{-\infty}^{0} \frac{d \alpha}{2 \alpha^{2}} \theta\left(y_{*}-x_{*}\right)\right] e^{-i \alpha\left(x_{\bullet}-y_{\bullet}\right)} \\
& \times\left\langle x_{\perp}\left|\hat{p} e^{-i \frac{\hat{p}_{\perp}^{2}}{\alpha s} x_{*}} p_{2}\left[x_{*}, y_{*}\right] e^{i \frac{\hat{p}_{\perp}^{2}}{\alpha s} y_{*}} \hat{p}\right| y_{\perp}\right\rangle
\end{aligned}
$$

Notice that to arrive at propagator (B.5), which is already in the shock-wave form, we did not need to perform the gauge rotation we performed in section 2.2, because expansion (B.1) assumes from the start a free propagation before and after the interaction with the external 
field, as can also be seen from its diagrammatic representation in figure 4. In other words, when we start drawing Feynman diagrams, like in figure 4, we are implicitly fixing a gauge for the external field.

\section{The $\left\{\hat{P}_{\bullet}, A_{*}\right\}$ term}

In section 3 we showed that the term $\left\{\hat{P}_{\bullet}, A_{*}\right\}$ is actually a sub-sub-eikonal correction. We now demonstrate the same thing following an alternative procedure. To this end, we notice that (for simplicity we omit the $+i \epsilon$ prescription from each $\frac{1}{\hat{p}^{2}+2 \alpha g A_{\bullet}}$ and the ${ }^{\wedge}$ symbol on top of operators)

$$
\begin{aligned}
& \frac{1}{p^{2}+2 \alpha g A_{\bullet}}\left\{P_{\bullet}, A_{*}\right\} \frac{1}{p^{2}+2 \alpha g A_{\bullet}} \\
& =\frac{1}{2 \alpha}\left\{A_{*}, \frac{1}{\hat{p}^{2}+2 \alpha g A_{\bullet}}\right\}+\frac{1}{2 \alpha} \frac{1}{p^{2}+2 \alpha g A_{\bullet}}\left\{p_{\perp}^{2}, A_{*}\right\} \frac{1}{p^{2}+2 \alpha g A_{\bullet}} \\
& =\frac{1}{2 \alpha}\left\{A_{*}, \frac{1}{p^{2}+2 \alpha g A_{\bullet}}\right\}+O\left(\lambda^{-2}\right) .
\end{aligned}
$$

Thus, we are left with sub-eikonal corrections with the $A_{*}$ (a gauge dependent term) only at the edges of the gauge link. When we use the scalar propagator in intermediate steps with the terms (C.1), we will have terms like

$$
\begin{aligned}
& \frac{1}{p^{2}+2 \alpha g A_{\bullet}}\left\{A_{*}, i g F_{i \bullet}\right\} \frac{1}{p^{2}+2 \alpha g A_{\bullet}} \\
= & \frac{1}{p^{2}+2 \alpha g A_{\bullet}}\left(P_{i} P_{\bullet} A_{*}-A_{*} P_{\bullet} P_{i}\right) \frac{1}{p^{2}+2 \alpha g A_{\bullet}}+\frac{1}{2 \alpha} \frac{1}{p^{2}+2 \alpha g A_{\bullet}} A_{*} P_{i}-\frac{1}{2 \alpha} P_{i} A_{*} \frac{1}{p^{2}+2 \alpha g A_{\bullet}} \\
& +\frac{1}{2 \alpha} \frac{1}{p^{2}+2 \alpha g A_{\bullet}} A_{*} P_{i} p_{\perp}^{2} \frac{1}{p^{2}+2 \alpha g A_{\bullet}}-\frac{1}{2 \alpha} \frac{1}{p^{2}+2 \alpha g A_{\bullet}} p_{\perp}^{2} P_{i} A_{*} \frac{1}{p^{2}+2 \alpha g A_{\bullet}} \\
= & \frac{1}{2 \alpha} \frac{1}{p^{2}+2 \alpha g A_{\bullet}} A_{*} P_{i}-\frac{1}{2 \alpha} P_{i} A_{*} \frac{1}{p^{2}+2 \alpha g A_{\bullet}}+O\left(\lambda^{-2}\right) .
\end{aligned}
$$

So, once again, we arrived at an expression which has the non gauge-invariant terms only at the edges of the gauge links and they could be eliminated by a proper gauge choice since are outside the shock-wave. To arrive at eq. (C.2), we noticed that the term

$$
\frac{1}{2 \alpha} \frac{1}{p^{2}+2 \alpha g A_{\bullet}} A_{*} P_{i} p_{\perp}^{2} \frac{1}{p^{2}+2 \alpha g A_{\bullet}}-\frac{1}{2 \alpha} \frac{1}{p^{2}+2 \alpha g A_{\bullet}} p_{\perp}^{2} P_{i} A_{*} \frac{1}{p^{2}+2 \alpha g A_{\bullet}}
$$

is sub-sub-eikonal and therefore can be disregarded. Moreover, we used

$$
\begin{aligned}
\left\langle x\left|\frac{1}{p^{2}+2 \alpha g A_{\bullet}} A_{*} P_{\bullet} P_{i} \frac{1}{p^{2}+2 \alpha g A_{\bullet}}\right| y\right\rangle \\
=\int d z\left\langle x\left|\frac{1}{p^{2}+2 \alpha g A_{\bullet}}\right| z\right\rangle A_{*}\left(z_{*}, z_{\perp}\right) i D_{\bullet}^{z} i D_{i}^{z}\left\langle z\left|\frac{1}{p^{2}+2 \alpha g A_{\bullet}}\right| y\right\rangle \\
=\left[-\int_{0}^{+\infty} \frac{d \alpha}{4 \alpha^{2}} \theta\left(x_{*}-y_{*}\right)+\int_{-\infty}^{0} \frac{d \alpha}{4 \alpha^{2}} \theta\left(y_{*}-x_{*}\right)\right] e^{-i \alpha\left(x_{\bullet}-y_{\bullet}\right)} \\
\quad \times \int_{y_{*}}^{x_{*}} d \frac{2}{s} z_{1 *}\left\langle x_{\perp}\left|e^{-i \frac{\hat{p}_{\perp}^{2}}{\alpha s} x_{*}}\left[x_{*}, z_{*}\right] e^{i \frac{\hat{p}_{\perp}^{2}}{\alpha s} z_{*}} A_{*}\left(z_{*}\right) i D_{\bullet}^{z} i D_{i}^{z} e^{-i \frac{\hat{p}_{\perp}^{2}}{\alpha s} z_{*}}\left[z_{*}, y_{*}\right] e^{i \frac{\hat{p}_{\perp}^{2}}{\alpha s} y_{*}}\right| y_{\perp}\right\rangle
\end{aligned}
$$




$$
\begin{aligned}
= & {\left[-\int_{0}^{+\infty} \frac{d \alpha}{4 \alpha^{2}} \theta\left(x_{*}-y_{*}\right)+\int_{-\infty}^{0} \frac{d \alpha}{4 \alpha^{2}} \theta\left(y_{*}-x_{*}\right)\right] e^{-i \alpha\left(x_{\bullet}-y_{\bullet}\right)} } \\
& \times(-g) \int_{y_{*}}^{x_{*}} d \frac{2}{s} z_{1 *}\left\langle x_{\perp}\right| e^{-i \frac{\hat{p}_{\perp}^{2}}{\alpha s} x_{*}}\left[x_{*}, z_{*}\right] e^{i \frac{\hat{p}_{\perp}^{2}}{\alpha s} z_{*}} A_{*}\left(z_{*}\right) i D_{\bullet}^{z} e^{-i \frac{\hat{p}_{\perp}^{2}}{\alpha s} z_{*}} \\
& \times \int_{y_{*}}^{z_{*}} d \frac{2}{s} z_{*}^{\prime}\left[z_{*}, z_{*}^{\prime}\right] F_{i \bullet}\left[z_{*}^{\prime}, y_{*}\right] e^{i \frac{\hat{p}_{\perp}^{2}}{\alpha s} y_{*}}\left|y_{\perp}\right\rangle \\
= & {\left[-\int_{0}^{+\infty} \frac{d \alpha}{4 \alpha^{2}} \theta\left(x_{*}-y_{*}\right)+\int_{-\infty}^{0} \frac{d \alpha}{4 \alpha^{2}} \theta\left(y_{*}-x_{*}\right)\right] e^{-i \alpha\left(x_{\bullet}-y_{\bullet}\right)} } \\
& \times(-g) \int_{y_{*}}^{x_{*}} d \frac{2}{s} z_{1 *}\left\langle x_{\perp}\right| e^{-i \frac{\hat{p}_{\perp}^{2}}{\alpha s} x_{*}}\left[x_{*}, z_{*}\right]\left(A_{*}\left(z_{*}\right)+\frac{i z_{*}}{\alpha s}\left[\hat{p}^{2}, A_{*}\right]\right)\left(\frac{i z_{*}}{\alpha s}\left[\hat{p}_{\perp}^{2}, A_{\bullet}\right]+\frac{\hat{p}_{\perp}^{2}}{2 \alpha}+i D_{\bullet}^{z}\right) \\
& \times \int_{y_{*}}^{z_{*}} d \frac{2}{s} z_{*}^{\prime}\left[z_{*}, z_{*}^{\prime}\right] F_{i \bullet}\left[z_{*}^{\prime}, y_{*}\right] e^{i \frac{\hat{p}_{\perp}^{2}}{\alpha s} y_{*}}\left|y_{\perp}\right\rangle=O\left(\lambda^{-2}\right),
\end{aligned}
$$

where we used the identity $\left(i \partial_{\bullet}^{x}+g A_{\bullet}\left(x_{*}\right)\right)\left[x_{*}, z_{*}\right]=0$. Clearly, the advantage of the procedure adopted in section 3 is that one does not have to deal with non gauge-invariant terms even though they are at the edges of the gauge links.

\section{Some calculation details of the sub-eikonal corrections to the quark propagator}

In this section we present some details of the calculation to obtain eq. (4.4). Our starting point is eq. (4.3) which we report here

$$
\begin{aligned}
\operatorname{Pexp} & \left\{i g \int_{y_{*}}^{x_{*}} d \frac{2}{s} \omega_{*} e^{i \frac{\hat{p}_{\perp}^{2}}{\alpha s} \omega_{*}}\left(A \bullet\left(\omega_{*}\right)+\frac{B\left(\omega_{*}\right)}{2 \alpha}+\frac{i}{2 \alpha} \frac{2}{s} F_{\bullet i}\left(\omega_{*}\right) p_{2} \gamma^{i}\right) e^{-i \frac{\hat{p}_{\perp}^{2}}{\alpha s} \omega_{*}}\right\} \\
= & {\left[x_{*}, y_{*}\right]+i g \int_{y_{*}}^{x_{*}} d \frac{2}{s} \omega_{*}\left[x_{*}, \omega_{*}\right]\left(\frac{O\left(\omega_{*}\right)}{2 \alpha}+i \frac{\omega_{*}}{\alpha s}\left[p_{\perp}^{2}, A_{\bullet}\right]\right)\left[\omega_{*}, y_{*}\right] } \\
& +\frac{i g}{2 \alpha} \int_{y_{*}}^{x_{*}} d \frac{2}{s} \omega_{*}\left[x_{*}, \omega_{*}\right]\left(\frac{4}{s^{2}} F_{\bullet *} \sigma_{* \bullet}+\frac{1}{2} F_{i j} \sigma^{i j}\right)\left[\omega_{*}, y_{*}\right] \\
& +i g \int_{y_{*}}^{x_{*}} d \frac{2}{s} \omega_{*}\left[x_{*}, \omega_{*}\right]\left(\frac{i}{2 \alpha} \frac{2}{s} F_{\bullet i} \not p_{2} \gamma^{i}+i \frac{\omega_{*}}{\alpha s}\left[p_{\perp}^{2}, \frac{i}{2 \alpha} \frac{2}{s} F_{\bullet} \not p_{2} \gamma^{i}\right]\right)\left[\omega_{*}, y_{*}\right] \\
& +(i g)^{2} \int_{y_{*}}^{x_{*}} d \frac{2}{s} \omega_{*} \int_{y_{*}}^{\omega_{*}} d \frac{2}{s} \omega_{*}^{\prime}\left[x_{*}, \omega_{*}\right]\left(\frac{B\left(\omega_{*}\right)}{2 \alpha}+i \frac{\omega_{*}}{\alpha s}\left[p_{\perp}^{2}, A_{\bullet}\right]\right)\left[\omega_{*}, \omega_{*}^{\prime}\right] \frac{i}{2 \alpha} \frac{2}{s} F_{\bullet i}\left(\omega_{*}^{\prime}\right) p_{2} \gamma^{i}\left[\omega_{*}^{\prime}, y_{*}\right] \\
& +(i g)^{2} \int_{y_{*}}^{x_{*}} d \frac{2}{s} \omega_{*} \int_{y_{*}}^{\omega_{*}} d \frac{2}{s} \omega_{*}^{\prime}\left[x_{*}, \omega_{*}\right] \frac{i}{2 \alpha} \frac{2}{s} F_{\bullet}\left(\omega_{*}\right) \not p_{2} \gamma^{i}\left[\omega_{*}, \omega_{*}^{\prime}\right]\left(\frac{B\left(\omega_{*}^{\prime}\right)}{2 \alpha}+i \frac{\omega_{*}^{\prime}}{\alpha s}\left[p_{\perp}^{2}, A_{\bullet}\right]\right)\left[\omega_{*}^{\prime}, y_{*}\right] \\
& +O\left(\lambda^{-2}\right) .
\end{aligned}
$$

Our goal is to rewrite (D.1) in a gauge invariant form. To this end, we divide the righthand-side of eq. (D.1) in four pieces, simplify them separately, and then sum them up. 
Using result (3.7), the first piece in (4.3) reduces to

$$
\begin{aligned}
& {\left[x_{*}, y_{*}\right]+i g \int_{y_{*}}^{x_{*}} d \frac{2}{s} \omega_{*}\left[x_{*}, \omega_{*}\right]\left(\frac{O\left(\omega_{*}\right)}{2 \alpha}+i \frac{\omega_{*}}{\alpha s}\left[p_{\perp}^{2}, A_{\bullet}\right]\right)\left[\omega_{*}, y_{*}\right]} \\
& +\frac{i g}{2 \alpha} \int_{y_{*}}^{x_{*}} d \frac{2}{s} \omega_{*}\left[x_{*}, \omega_{*}\right]\left(\frac{4}{s^{2}} F_{\bullet *} \sigma_{* \bullet}+\frac{1}{2} F_{i j} \sigma^{i j}\right)\left[\omega_{*}, y_{*}\right] \\
& =\left[x_{*}, y_{*}\right]+\frac{i g}{2 \alpha}\left[\frac{2}{s} x_{*}\left(\left\{P_{i}, A^{i}\left(x_{*}\right)\right\}-g A_{i}\left(x_{*}\right) A^{i}\left(x_{*}\right)\right)\left[x_{*}, y_{*}\right]\right. \\
& \quad-\left[x_{*}, y_{*}\right] \frac{2}{s} y_{*}\left(\left\{P_{i}, A^{i}\left(y_{*}\right)\right\}-g A_{i}\left(y_{*}\right) A^{i}\left(y_{*}\right)\right) \\
& +\int_{y_{*}}^{x_{*}} d \frac{2}{s} \omega_{*}\left(\left\{P^{i},\left[x_{*}, \omega_{*}\right] \frac{2}{s} \omega_{*} F_{i \bullet}\left(\omega_{*}\right)\left[\omega_{*}, y_{*}\right]\right\}\right. \\
& \left.\left.+g \int_{\omega_{*}}^{x_{*}} d \frac{2}{s} \omega_{*}^{\prime} \frac{2}{s}\left(\omega_{*}-\omega_{*}^{\prime}\right)\left[x_{*}, \omega_{*}^{\prime}\right] F^{i} \bullet\left[\omega_{*}^{\prime}, \omega_{*}\right] F_{i \bullet}\left[\omega_{*}, y_{*}\right]\right)\right] \\
& \quad+\frac{i g}{2 \alpha} \int_{y_{*}}^{x_{*}} d \frac{2}{s} \omega_{*}\left[x_{*}, \omega_{*}\right]\left(\frac{4}{s^{2}} F_{\bullet *} \sigma_{* \bullet}+\frac{1}{2} F_{i j} \sigma^{i j}\right)\left[\omega_{*}, y_{*}\right] .
\end{aligned}
$$

Next, making use of the following identity

$$
\begin{aligned}
{\left[p_{\perp}^{2}, F_{j \bullet}\right]=} & g\left(\left\{P^{i}, A_{i}\right\}-g A^{i} A_{i}\right) F_{j \bullet} \\
& -g F_{j \bullet}\left(\left\{P^{i}, A_{i}\right\}-g A^{i} A_{i}\right)-\left\{P^{i},\left(i D_{i} F_{j \bullet}\right)\right\},
\end{aligned}
$$

the second piece reduces to

$$
\begin{aligned}
& i g \int_{y_{*}}^{x_{*}} d \frac{2}{s} \omega_{*}\left[x_{*}, \omega_{*}\right]\left(\frac{i}{2 \alpha} \frac{2}{s} F_{\bullet} \not p_{2} \gamma^{i}+i \frac{\omega_{*}}{\alpha s}\left[p_{\perp}^{2}, \frac{i}{2 \alpha} \frac{2}{s} F_{\bullet i} \not p_{2} \gamma^{i}\right]\right)\left[\omega_{*}, y_{*}\right] \\
& =-\frac{i}{2 \alpha} \frac{2}{s} \not p_{2}\left(D_{\perp}\left[x_{*}, y_{*}\right]\right)-\frac{i g}{4 \alpha^{2}} \gamma^{j} \frac{2}{s} \not p_{2} \int_{y_{*}}^{x_{*}} d \frac{2}{s} \omega_{*}\left[\frac{2}{s} \omega_{*}\left[x_{*}, \omega_{*}\right]\right. \\
& \quad \times\left(g\left(\left\{P^{i}, A_{i}\right\}-g A^{i} A_{i}\right) F_{j \bullet}-g F_{j \bullet}\left(\left\{P^{i}, A_{i}\right\}-g A^{i} A_{i}\right)\right)\left[\omega_{*}, y_{*}\right] \\
& \quad-\frac{2}{s} \omega_{*}\left\{P^{i},\left[x_{*}, \omega_{*}\right]\left(i D_{i} F_{j \bullet}\right)\left[\omega_{*}, y_{*}\right]\right\}-\frac{2}{s} \omega_{*}\left[x_{*}, \omega_{*}\right]\left(i D^{i} F_{j \bullet}\right)\left(i \mathfrak{D}_{i}\left[\omega_{*}, y_{*}\right]\right) \\
& \left.\quad+\frac{2}{s} \omega_{*}\left(i \mathfrak{D}^{i}\left[x_{*}, \omega_{*}\right]\right)\left(i D_{i} F_{j \bullet}\right)\left[\omega_{*}, y_{*}\right]\right] .
\end{aligned}
$$

Note that identity (D.3) highlights the importance of the transverse fields at the edges of the gauge-link in the scalar propagator (3.7). These terms can be set to zero only when they are at the point $x_{*}$ and $y_{*}$, but when we use the scalar propagator as an intermediate step, these terms will not always be at the edges. So, setting them to zero once and for all, will lead to a wrong result. 
We now turn our attention to the third piece. After some algebra it reduces to

$$
\begin{aligned}
& (i g)^{2} \int_{y_{*}}^{x_{*}} d \frac{2}{s} \omega_{*} \int_{y_{*}}^{\omega_{*}} d \frac{2}{s} \omega_{*}^{\prime}\left[x_{*}, \omega_{*}\right]\left(\frac{B\left(\omega_{*}\right)}{2 \alpha}+i \frac{\omega_{*}}{\alpha s}\left[p_{\perp}^{2}, A_{\bullet}\right]\right)\left[\omega_{*}, \omega_{*}^{\prime}\right] \frac{i}{2 \alpha} \frac{2}{s} F_{\bullet}\left(\omega_{*}^{\prime}\right) p_{2} \gamma^{i}\left[\omega_{*}^{\prime}, y_{*}\right] \\
& =\left(\frac{i g}{2 \alpha}\right)^{2} \int_{y_{*}}^{x_{*}} d \frac{2}{s} \omega_{*}^{\prime}\left(\frac{2}{s} x_{*}\left(\left\{P_{i}, A^{i}\left(x_{*}\right)\right\}-g A_{i} A^{i}\right)\left[x_{*}, \omega_{*}^{\prime}\right]\right. \\
& \left.\quad-\left[x_{*}, \omega_{*}^{\prime}\right] \frac{2}{s} \omega_{*}^{\prime}\left(\left\{P_{i}, A^{i}\left(\omega_{*}^{\prime}\right)\right\}-g A_{i} A^{i}\right)\right) i \frac{2}{s} F_{i \bullet} \gamma^{i} \not p_{2}\left[\omega_{*}^{\prime}, y_{*}\right] \\
& \quad+\frac{g}{4 \alpha^{2}} \frac{2}{s} p_{2} \int_{y_{*}}^{x_{*}} d \frac{2}{s} \omega_{*}\left[x_{*}, \omega_{*}\right] \mathcal{B}_{1}\left(\mathscr{D}_{\perp}\left[\omega_{*}, y_{*}\right]\right) \\
& \quad+\frac{g^{2}}{4 \alpha^{2}} \frac{2}{s} p_{2} \int_{y_{*}}^{x_{*}} d \frac{2}{s} \omega_{*}^{\prime} \int_{y_{*}}^{\omega_{*}^{\prime}} d \frac{2}{s} \omega_{*} \frac{2}{s} \omega_{*}\left[x_{*}, \omega_{*}^{\prime}\right] F_{\bullet}^{i}\left[\omega_{*}^{\prime}, \omega_{*}\right] F_{i \bullet}\left(\mathscr{D}_{\perp}\left[\omega_{*}, y_{*}\right]\right) \\
& \quad+\frac{g}{4 \alpha^{2}} \frac{2}{s} p_{2} \int_{y_{*}}^{x_{*}} d \frac{2}{s} \omega_{*}\left[\left\{P^{i},\left[x_{*}, \omega_{*}\right] \frac{2}{s} \omega_{*} F_{i \bullet} \bullet \mathscr{D}_{\perp}\left[\omega_{*}, y_{*}\right]\right)\right\} \\
& \left.\quad+\left[x_{*}, \omega_{*}\right] \frac{2}{s} \omega_{*} F_{i \bullet}\left(i \mathfrak{D}^{i}\left(\mathscr{D}_{\perp}\left[\omega_{*}, y_{*}\right]\right)\right)\right] .
\end{aligned}
$$

Finally, let us consider the forth piece

$$
\begin{aligned}
& (i g)^{2} \int_{y_{*}}^{x_{*}} d \frac{2}{s} \omega_{*} \int_{y_{*}}^{\omega_{*}} d \frac{2}{s} \omega_{*}^{\prime}\left[x_{*}, \omega_{*}\right] \frac{i}{2 \alpha} \frac{2}{s} F_{\bullet}\left(\omega_{*}\right) p_{2} \gamma^{i}\left[\omega_{*}, \omega_{*}^{\prime}\right]\left(\frac{B\left(\omega_{*}^{\prime}\right)}{2 \alpha}+i \frac{\omega_{*}^{\prime}}{\alpha s}\left[p_{\perp}^{2}, A_{\bullet}\right]\right)\left[\omega_{*}^{\prime}, y_{*}\right] \\
& =\left(\frac{i g}{2 \alpha}\right)^{2} \int_{y_{*}}^{x_{*}} d \frac{2}{s} \omega_{*}\left[[ x _ { * } , \omega _ { * } ] i \frac { 2 } { s } F _ { i \bullet } \gamma ^ { i } p _ { 2 } \left(\frac{2}{s} \omega_{*}\left(\left\{P_{i}, A^{i}\right\}-g A_{i} A^{i}\right)\left[\omega_{*}, y_{*}\right]\right.\right. \\
& \left.-\frac{2}{s} y_{*}\left[\omega_{*}, y_{*}\right]\left(\left\{P_{i}, A^{i}\right\}-g A_{i} A^{i}\right)\right)+\frac{2}{s} \not p_{2}\left(i \mathfrak{D}^{i}\left(\mathfrak{D}_{\perp}\left[x_{*}, \omega_{*}\right]\right)\right) \frac{2}{s} \omega_{*} F_{i \bullet}\left[\omega_{*}, y_{*}\right] \\
& -\left\{P^{i},\left(D_{\perp}\left[x_{*}, \omega_{*}\right]\right) \frac{2}{s} \omega_{*} F_{i \bullet}\left[\omega_{*}, y_{*}\right]\right\}-\left(\mathscr{D}_{\perp}\left[x_{*}, \omega_{*}\right]\right) \mathcal{B}_{1}\left[\omega_{*}, y_{*}\right] \\
& \left.+g \int_{\omega_{*}}^{x_{*}} d \frac{2}{s} \omega_{*}^{\prime} \frac{2}{s} \omega_{*}^{\prime}\left(\mathscr{D}_{\perp}\left[x_{*}, \omega_{*}^{\prime}\right]\right) F^{i} \bullet\left[\omega_{*}^{\prime}, \omega_{*}\right] F_{i \bullet}\left[\omega_{*}, y_{*}\right]\right] .
\end{aligned}
$$

We can now sum the four terms (D.2), (D.4), (D.5) and (D.6) and, since we are interested in the shock-wave limit, we can disregard the fields that are at the edges of the gauge-links, that is at points $x_{*}$ and $y_{*}$. After some algebra, we obtain

$$
\begin{aligned}
& \operatorname{Pexp}\left\{i g \int_{y_{*}}^{x_{*}} d \frac{2}{s} \omega_{*} e^{i \frac{\hat{p}_{\perp}^{2}}{\alpha s} \omega_{*}}\left(A_{\bullet}\left(\omega_{*}\right)+\frac{B\left(\omega_{*}\right)}{2 \alpha}+\frac{i}{2 \alpha} \frac{2}{s} F_{\bullet}\left(\omega_{*}\right) \not p_{2} \gamma^{i}\right) e^{-i \frac{\hat{p}_{\perp}^{2}}{\alpha s} \omega_{*}}\right\} \\
& =\left(\left(1-\frac{1}{2 \alpha} \frac{2}{s} \not p_{2} i D_{\perp}\right)\left[x_{*}, y_{*}\right]+\frac{i g}{2 \alpha} \int_{y_{*}}^{x_{*}} d \frac{2}{s} \omega_{*}\left[x_{*}, \omega_{*}\right] \mathcal{B}_{1}\left[\omega_{*}, y_{*}\right]\right) \\
& +\frac{1}{4 \alpha^{2}} \int_{y_{*}}^{x_{*}} d \frac{2}{s} z_{*}\left[\left(i \mathfrak{P}_{\perp} \frac{2}{s} \not p_{2}\left[x_{*}, z_{*}\right]\right) i g \mathcal{B}_{1}\left[z_{*}, y_{*}\right]+\left[x_{*}, z_{*}\right] i g \mathcal{B}_{1}\left(i \mathfrak{D}_{\perp} \frac{2}{s} \not p_{2}\left[z_{*}, y_{*}\right]\right)\right] \\
& +\frac{i g}{2 \alpha}\left[\int _ { y _ { * } } ^ { x _ { * } } d \frac { 2 } { s } \omega _ { * } \left(\left\{p^{i},\left[x_{*}, \omega_{*}\right] \frac{2}{s} \omega_{*} F_{i \bullet}\left(\omega_{*}\right)\left[\omega_{*}, y_{*}\right]\right\}\right.\right. \\
& \left.\left.+g \int_{\omega_{*}}^{x_{*}} d \frac{2}{s} \omega_{*}^{\prime} \frac{2}{s}\left(\omega_{*}-\omega_{*}^{\prime}\right)\left[x_{*}, \omega_{*}^{\prime}\right] F^{i} \bullet\left[\omega_{*}^{\prime}, \omega_{*}\right] F_{i \bullet}\left[\omega_{*}, y_{*}\right]\right)\right]-\frac{i g}{4 \alpha^{2}} \gamma^{j} \frac{2}{s} \not p_{2} \int_{y_{*}}^{x_{*}} d \frac{2}{s} \omega_{*} \frac{2}{s} \omega_{*}
\end{aligned}
$$




$$
\begin{aligned}
& \times\left[\left(i \mathfrak{D}^{i}\left[x_{*}, \omega_{*}\right]\right)\left(i D_{i} F_{j \bullet}\right)\left[\omega_{*}, y_{*}\right]-\left[x_{*}, \omega_{*}\right]\left(i D^{i} F_{j \bullet}\right)\left(i \mathfrak{D}_{i}\left[\omega_{*}, y_{*}\right]\right)-\left\{p^{i},\left[x_{*}, \omega_{*}\right]\left(i D_{i} F_{j \bullet}\right)\left[\omega_{*}, y_{*}\right]\right\}\right] \\
& +\frac{g}{4 \alpha^{2}} \frac{2}{s} \not p_{2} \int_{y_{*}}^{x_{*}} d \frac{2}{s} \omega_{*}\left[\frac{2}{s} \omega_{*}\left(\mathscr{D}_{\perp}\left[x_{*}, \omega_{*}\right]\right) F_{i \bullet}\left(i \mathfrak{D}^{i}\left[\omega_{*}, y_{*}\right]\right)-\frac{2}{s} \omega_{*}\left(i \mathfrak{D}^{i}\left[x_{*}, \omega_{*}\right]\right) F_{i \bullet}\left(\mathscr{D}_{\perp}\left[\omega_{*}, y_{*}\right]\right)\right. \\
& +\left\{p^{i},\left[x_{*}, \omega_{*}\right] \frac{2}{s} \omega_{*} F_{i \bullet}\left(\mathscr{D}_{\perp}\left[\omega_{*}, y_{*}\right]\right)\right\}+\left\{p^{i},\left(\mathscr{P}_{\perp}\left[x_{*}, \omega_{*}\right]\right) \frac{2}{s} \omega_{*} F_{i \bullet}\left[\omega_{*}, y_{*}\right]\right\} \\
& \left.+\left[x_{*}, \omega_{*}\right] \frac{2}{s} \omega_{*} F_{i \bullet} i \mathfrak{D}^{i}\left(\mathscr{D}_{\perp}\left[\omega_{*}, y_{*}\right]\right)-\left(i \mathfrak{D}^{i}\left(\mathfrak{D}_{\perp}\left[x_{*}, \omega_{*}\right]\right)\right) \frac{2}{s} \omega_{*} F_{i \bullet}\left[\omega_{*}, y_{*}\right]\right]+O\left(\lambda^{-2}\right),
\end{aligned}
$$

where we have defined $\mathcal{B}_{1} \equiv \frac{4}{s^{2}} F_{\bullet *} \sigma_{* \bullet}+\frac{1}{2} F_{i j} \sigma^{i j}$.

Note that, to get to eq. (D.7) we could have started, alternatively, from the following type of expansion

$$
\begin{aligned}
\left\langle x\left|P \frac{i}{P^{2}+\frac{g}{2} F_{\mu \nu} \sigma^{\mu \nu}}\right| y\right\rangle & \\
= & \left\langlex | \not | P \left[\frac{i}{P^{2}}-\frac{i}{P^{2}}\left(i g \frac{2}{s} F_{i \bullet} \gamma^{i} p_{2}+g \mathcal{B}_{1}\right) \frac{1}{P^{2}}\right.\right. \\
& \left.+\frac{i}{P^{2}}\left(i g \frac{2}{s} F_{i \bullet} \gamma^{i} \not p_{2}+g \mathcal{B}_{1}\right) \frac{1}{P^{2}}\left(i g \frac{2}{s} F_{i \bullet} \gamma^{i} p_{2}+g \mathcal{B}_{1}\right) \frac{1}{P^{2}}\right]|y\rangle \\
= & i \not D\langle x|\left[\frac{i}{p^{2}+2 g \alpha A_{\bullet}+g O}-\frac{i}{p^{2}+2 g \alpha A_{\bullet}+g O} i g \frac{2}{s} F_{i \bullet} \gamma^{i} \not p_{2} \frac{1}{p^{2}+2 g \alpha A_{\bullet}+g O}\right. \\
& -\frac{i}{p^{2}+2 g \alpha A_{\bullet}} g \mathcal{B}_{1} \frac{1}{p^{2}+2 g \alpha A_{\bullet}}+\frac{i}{p^{2}+2 g \alpha A_{\bullet}} i g \frac{2}{s} F_{\bullet} \gamma^{i} \not p_{2} \frac{1}{p^{2}+2 g \alpha A_{\bullet}} \mathcal{B}_{1} \frac{1}{p^{2}+2 g \alpha A_{\bullet}} \\
& \left.+\frac{i}{p^{2}+2 g \alpha A_{\bullet}} \mathcal{B}_{1} \frac{1}{p^{2}+2 g \alpha A_{\bullet}} i g \frac{2}{s} F_{i \bullet} \gamma^{i} p_{2} \frac{1}{p^{2}+2 g \alpha A_{\bullet}}\right]|y\rangle,
\end{aligned}
$$

and, substituting the scalar propagator with sub-eikonal corrections, eq. (3.7), for each $\frac{i}{p^{2}+2 g \alpha A_{\bullet}+g O}$ factor, and the eikonal scalar propagator, eq. (2.9), for each $\frac{i}{p^{2}+2 g \alpha A_{\bullet}}$ factor, we would get to the same gauge invariant expression, eq. (D.7), with the help of steps similar to eqs. (D.2), (D.4), (D.5) and (D.6).

\section{E Quark and scalar propagators with one end-point in the external field}

\section{E.1 Scalar propagator}

In section 3 we derived the scalar propagator for the shock-wave case, that is, for the case in which the particle starts and ends its propagation outside the shock-wave as shown in figure 1. We expanded around a point which is in the middle of the external field, eq. (3.5). We are now interested in the scalar propagator for a particle that starts or ends its propagation inside the external field. We consider the case in which the point $x_{*}$ is in the shock-wave, i.e. inside the interval in which the filed strength tensor is different then zero. In this case, expansion (2.6) can be written as

$$
\begin{aligned}
\left\langle x\left|\frac{i}{P^{2}+i \epsilon}\right| y\right\rangle= & {\left[\int_{0}^{+\infty} \frac{d \alpha}{2 \alpha} \theta\left(x_{*}-y_{*}\right)-\int_{-\infty}^{0} \frac{d \alpha}{2 \alpha} \theta\left(y_{*}-x_{*}\right)\right] e^{-i \alpha\left(x_{\bullet}-y_{\bullet}\right)} } \\
& \times\left\langle x_{\perp}\right| \operatorname{Pexp}\left\{i g \int_{y_{*}}^{x_{*}} d \frac{2}{s} \omega_{*} e^{i \frac{\hat{p}_{\perp}^{2}}{\alpha s}\left(\omega_{*}-x_{*}\right)}\left(A \bullet\left(\omega_{*}\right)+\frac{O\left(\omega_{*}\right)}{2 \alpha}\right) e^{-i \frac{\hat{p}_{\perp}^{2}}{\alpha s}\left(\omega_{*}-x_{*}\right)}\right\} \\
& \times e^{-i \frac{\hat{p}_{\perp}^{2}}{\alpha s}\left(x_{*}-y_{*}\right)}\left|y_{\perp}\right\rangle .
\end{aligned}
$$


In eq. (E.1), we observe that the coordinate $x_{*}$, which is the end point of the particle's propagation, is now in the path ordered exponential. This means that both, particle and external field end at $x_{*}$. We can now repeat the same steps we performed in the previous section and arrive at

$$
\begin{aligned}
\left\langle x\left|\frac{i}{P^{2}+i \epsilon}\right| y\right\rangle= & {\left[\int_{0}^{+\infty} \frac{d \alpha}{2 \alpha} \theta\left(x_{*}-y_{*}\right)-\int_{-\infty}^{0} \frac{d \alpha}{2 \alpha} \theta\left(y_{*}-x_{*}\right)\right] e^{-i \alpha\left(x_{\bullet}-y_{\bullet}\right)} } \\
& \times\left\langle x_{\perp}\right|\left\{\left[x_{*}, y_{*}\right]+\frac{i g}{2 \alpha}\left[\left[x_{*}, y_{*}\right] \frac{2}{s}\left(x_{*}-y_{*}\right)\left(\left\{P_{i}, A^{i}\left(y_{*}\right)\right\}-g A_{i}\left(y_{*}\right) A^{i}\left(y_{*}\right)\right)\right.\right. \\
& +\int_{y_{*}}^{x_{*}} d \frac{2}{s} \omega_{*}\left(\left\{P^{i},\left[x_{*}, \omega_{*}\right] \frac{2}{s}\left(\omega_{*}-x_{*}\right) F_{i \bullet}\left(\omega_{*}\right)\left[\omega_{*}, y_{*}\right]\right\}\right. \\
& \left.\left.\left.+g \int_{\omega_{*}}^{x_{*}} d \frac{2}{s} \omega_{*}^{\prime} \frac{2}{s}\left(\omega_{*}-\omega_{*}^{\prime}\right)\left[x_{*}, \omega_{*}^{\prime}\right] F^{i} \bullet\left[\omega_{*}^{\prime}, \omega_{*}\right] F_{i \bullet}\left[\omega_{*}, y_{*}\right]\right)\right]\right\} \\
& \times e^{-i \frac{\hat{p}_{\perp}^{2}}{\alpha s}\left(x_{*}-y_{*}\right)}\left|y_{\perp}\right\rangle+O\left(\lambda^{-2}\right) .
\end{aligned}
$$

We will now show that, since the particle ends its propagation inside the external field at point $x_{*}$, the non gauge-invariant terms, the transverse field $A_{i}$, will be only at point $y_{*}$. To this end we use

$$
\begin{aligned}
& \int_{y_{*}}^{x_{*}} d \frac{2}{s} \omega_{*}\left\{P^{i},\left[x_{*}, \omega_{*}\right] \frac{2}{s}\left(\omega_{*}-x_{*}\right) F_{i \bullet}\left[\omega_{*}, y_{*}\right]\right\} \\
& =\int_{y_{*}}^{x_{*}} d \frac{2}{s} \omega_{*}\left[\left[x_{*}, \omega_{*}\right] \frac{2}{s}\left(\omega_{*}-x_{*}\right)\left(i D^{i} F_{i \bullet}\left(\omega_{*}\right)\right)\left[\omega_{*}, y_{*}\right]+2\left[x_{*}, \omega_{*}\right] \frac{2}{s}\left(\omega_{*}-x_{*}\right) F_{i}\left(\omega_{*}\right)\left[\omega_{*}, y_{*}\right] P^{i}\right] \\
& \quad-g \int_{y_{*}}^{x_{*}} d \frac{2}{s} \omega_{*} \int_{\omega_{*}}^{x_{*}} d \frac{2}{s} \omega_{*}^{\prime}\left(\left[x_{*}, \omega_{*}^{\prime}\right] F_{i \bullet}\left(\omega_{*}^{\prime}\right)\left[\omega_{*}^{\prime}, \omega_{*}\right] \frac{2}{s}\left(\omega_{*}-x_{*}\right) F^{i} \bullet\left(\omega_{*}\right)\left[\omega_{*}, y_{*}\right]\right. \\
& \left.\quad+\left[x_{*}, \omega_{*}^{\prime}\right] \frac{2}{s}\left(\omega_{*}^{\prime}-x_{*}\right) F_{i \bullet}\left(\omega_{*}^{\prime}\right)\left[\omega_{*}^{\prime}, \omega_{*}\right] F^{i} \bullet\left(\omega_{*}\right)\left[\omega_{*}, y_{*}\right]\right)
\end{aligned}
$$

To arrive at eq. (E.3) we have pushed the operator $P_{i}$ to the right of the gauge link up to point $y_{*}$ which is the point outside the range in which $F_{\mu \nu}^{c l} \neq 0$. Hence, result (E.2) can be written as

$$
\begin{aligned}
\left\langle x\left|\frac{i}{P^{2}+i \epsilon}\right| y\right\rangle= & {\left[\int_{0}^{+\infty} \frac{d \alpha}{2 \alpha} \theta\left(x_{*}-y_{*}\right)-\int_{-\infty}^{0} \frac{d \alpha}{2 \alpha} \theta\left(y_{*}-x_{*}\right)\right] e^{-i \alpha\left(x_{\bullet}-y_{\bullet}\right)} } \\
& \times\left\langle x_{\perp}\right|\left\{\left[x_{*}, y_{*}\right]+\frac{i g}{2 \alpha}\left[\left[x_{*}, y_{*}\right] \frac{2}{s}\left(x_{*}-y_{*}\right)\left(\left\{P_{i}, A^{i}\left(y_{*}\right)\right\}-g A_{i}\left(y_{*}\right) A^{i}\left(y_{*}\right)\right)\right.\right. \\
& +\int_{y_{*}}^{x_{*}} d \frac{2}{s} \omega_{*}\left(\left[x_{*}, \omega_{*}\right] \frac{2}{s}\left(\omega_{*}-x_{*}\right)\left(i D^{i} F_{\bullet}\left(\omega_{*}\right)\right)\left[\omega_{*}, y_{*}\right]\right. \\
& -2 g \int_{\omega_{*}}^{x_{*}} d \frac{2}{s} \omega_{*}^{\prime}\left[x_{*}, \omega_{*}^{\prime}\right] \frac{2}{s}\left(\omega_{*}^{\prime}-x_{*}\right) F_{i \bullet}\left(\omega_{*}^{\prime}\right)\left[\omega_{*}^{\prime}, \omega_{*}\right] F^{i} \bullet\left(\omega_{*}\right)\left[\omega_{*}, y_{*}\right] \\
& \left.\left.\left.+2\left[x_{*}, \omega_{*}\right] \frac{2}{s}\left(\omega_{*}-x_{*}\right) F_{i}\left(\omega_{*}\right)\left[\omega_{*}, y_{*}\right] P^{i}\right)\right]\right\} e^{-i \frac{\hat{p}_{\perp}^{2}}{\alpha s}\left(x_{*}-y_{*}\right)}\left|y_{\perp}\right\rangle \\
& +O\left(\lambda^{-2}\right) .
\end{aligned}
$$




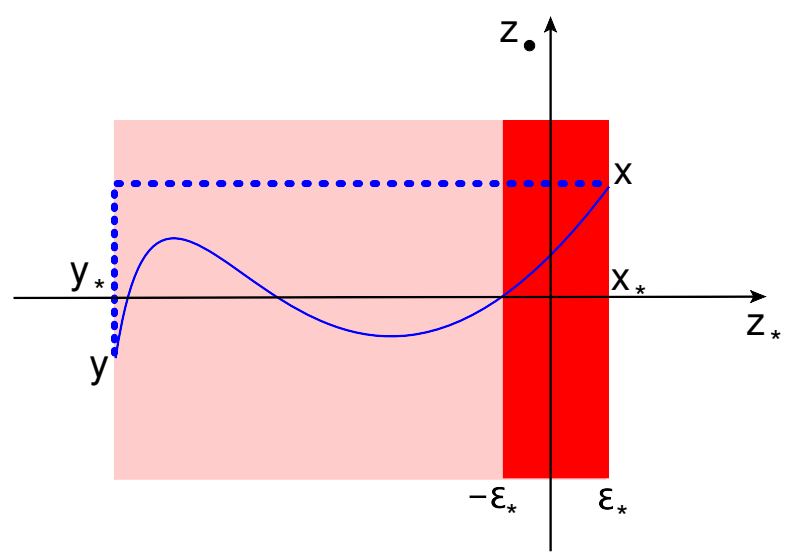

Figure 5. Particle starts its propagation within the shock-wave. In this case the pure gauge is only to the left of the shock-wave.

All non gauge-invariant terms in (E.4) are now only to the right of the gauge links, that is to the point $y_{*}$. This is opposite to what we have in eq. (3.7) which has the transverse field $A^{i}$ on both edges of the gauge link (i.e. at points $x_{*}$ and $y_{*}$ ). In the gauge-rotated field $A^{\Omega}$, we can set such non gauge-invariant terms to zero.

In a similar way we can expand with respect to $y_{\perp}+\frac{2}{s} p_{1} y_{*}$ and get

$$
\begin{aligned}
\left\langle x\left|\frac{i}{P^{2}+i \epsilon}\right| y\right\rangle=[ & \left.\int_{0}^{+\infty} \frac{d \alpha}{2 \alpha} \theta\left(x_{*}-y_{*}\right)-\int_{-\infty}^{0} \frac{d \alpha}{2 \alpha} \theta\left(y_{*}-x_{*}\right)\right] e^{-i \alpha\left(x_{\bullet}-y_{\bullet}\right)} \\
& \times\left\langle x_{\perp}\right| e^{-i \frac{\hat{p}_{\perp}^{2}}{\alpha s}\left(x_{*}-y_{*}\right)} \\
& \times\left\{\left[x_{*}, y_{*}\right]+\frac{i g}{2 \alpha}\left[\left(\left\{P_{i}, A^{i}\left(x_{*}\right)\right\}-g A_{i}\left(x_{*}\right) A^{i}\left(x_{*}\right)\right)\left[x_{*}, y_{*}\right] \frac{2}{s}\left(x_{*}-y_{*}\right)\right.\right. \\
& +\int_{y_{*}}^{x_{*}} d \frac{2}{s} \omega_{*}\left(-\left[x_{*}, \omega_{*}\right] \frac{2}{s}\left(\omega_{*}-y_{*}\right)\left(i D^{i} F_{i}\left(\omega_{*}\right)\right)\left[\omega_{*}, y_{*}\right]\right. \\
& +2 g \int_{\omega_{*}}^{x_{*}} d \frac{2}{s} \omega_{*}^{\prime}\left[x_{*}, \omega_{*}^{\prime}\right] F_{i}\left(\omega_{*}^{\prime}\right)\left[\omega_{*}^{\prime}, \omega_{*}\right] \frac{2}{s}\left(\omega_{*}-y_{*}\right) F_{\bullet}^{i}\left(\omega_{*}\right)\left[\omega_{*}, y_{*}\right] \\
& \left.\left.\left.+2 P^{i}\left[x_{*}, \omega_{*}\right] \frac{2}{s}\left(\omega_{*}-y_{*}\right) F_{i \bullet}\left(\omega_{*}\right)\left[\omega_{*}, y_{*}\right]\right)\right]\right\}\left|y_{\perp}\right\rangle+O\left(\lambda^{-2}\right) .
\end{aligned}
$$

In eq. (E.5) we have the non gauge-invariant terms only at point $x_{*}$

\section{E.2 Quark propagator}

In this section we derive the sub-eikonal corrections to the quark propagator with one end-point inside the external filed. To this end we need to use result (E.4). Let us suppose that $x_{\perp}+\frac{2}{s} p_{1} x_{*}$ is the end-point of the quark propagator in the external field as shown in figure 5. It is convenient to start from the following expression of the quark propagator

$$
\left\langle x\left|\frac{i}{\hat{p}+i \epsilon}\right| y\right\rangle=\left\langle x\left|\frac{i}{\hat{p}^{2}+2 \alpha g A_{\bullet}+g B+i \frac{2}{s} g F_{\bullet} \not p_{2} \gamma^{i}+i \epsilon} \hat{P}\right| y\right\rangle,
$$


and we need to include the point $x_{*}$ in the path-ordered exponential. So, we have

$$
\begin{aligned}
\left\langle x\left|\frac{i}{\hat{p}+i \epsilon}\right| y\right\rangle= & {\left[\int_{0}^{+\infty} \frac{d \alpha}{2 \alpha} \theta\left(x_{*}-y_{*}\right)-\int_{-\infty}^{0} \frac{d \alpha}{2 \alpha} \theta\left(y_{*}-x_{*}\right)\right] e^{-i \alpha\left(x_{\bullet}-y_{\bullet}\right)}\left\langle x_{\perp}\right| e^{-i \frac{\hat{p}_{\perp}^{2}}{\alpha s} x_{*}} } \\
& \times \operatorname{Pexp}\left\{i g \int_{y_{*}}^{x_{*}} d \frac{2}{s} \omega_{*} e^{i \frac{\hat{p}_{\perp}^{2}}{\alpha s} \omega_{*}}\left(A_{\bullet}\left(\omega_{*}\right)+\frac{B\left(\omega_{*}\right)}{2 \alpha}+\frac{i}{2 \alpha} \frac{2}{s} F_{\bullet}\left(\omega_{*}\right) \not p_{2} \gamma^{i}\right)\right. \\
& \left.\times e^{-i \frac{\hat{p}_{\perp}^{2}}{\alpha s} \omega_{*}}\right\} e^{i \frac{\hat{p}_{\perp}^{2}}{\alpha s} y_{*}}\left|y_{\perp}\right\rangle\left(-i \overleftarrow{\not} \overleftarrow{p}^{y}+g \frac{2}{s} A_{\bullet}\left(y_{*}, y_{\perp}\right) p_{2}+g A_{\perp}\left(y_{*}, y_{\perp}\right)\right) .
\end{aligned}
$$

We also need

$$
\begin{aligned}
& \left\langle z_{\perp}\left|e^{i \frac{\hat{p}_{\perp}^{2}}{\alpha s}\left(y_{*}-x_{*}\right)}\right| y_{\perp}\right\rangle e^{-i \alpha\left(x_{\bullet}-y_{\bullet}\right)}\left(i \overleftarrow{\not} y+g \frac{2}{s} \not p_{2} A \bullet\left(y_{*}, y_{\perp}\right)+A\left(y_{*}\right)\right) \\
& =\left\langle z_{\perp}\right|\left(\frac{1}{\alpha s} \not p p_{2} \not p-\frac{2}{s} \not p_{2} i \overleftarrow{D}_{\bullet}^{y}-i \frac{\left(x_{*}-y_{*}\right)}{\alpha s}\left[\hat{p}_{\perp}^{2}, g \frac{2}{s} \not p_{2} A_{\bullet}\left(y_{*}\right)\right]+A_{\perp}\left(y_{*}\right)\right) \\
& \times e^{i \frac{\hat{p}_{\perp}^{2}}{\alpha s}\left(y_{*}-x_{*}\right)}\left|y_{\perp}\right\rangle e^{-i \alpha\left(x_{\bullet}-y_{\bullet}\right)} \text {. }
\end{aligned}
$$

At this point the procedure we have to adopt is the same as the one performed in the previous section. Therefore, we arrive at

$$
\begin{aligned}
& \left\langle x\left|\frac{i}{P+i \epsilon}\right| y\right\rangle=\left[\int_{0}^{+\infty} \frac{d \alpha}{2 \alpha} \theta\left(x_{*}-y_{*}\right)-\int_{-\infty}^{0} \frac{d \alpha}{2 \alpha} \theta\left(y_{*}-x_{*}\right)\right] e^{-i \alpha\left(x_{\bullet}-y_{\bullet}\right)} \\
& \times\left\langle x_{\perp}\right|\left\{\left(\left[x_{*}, y_{*}\right] \not p-g \int_{y_{*}}^{x_{*}} d \frac{2}{s} \omega_{*} \gamma^{i}\left[x_{*}, \omega_{*}\right] F_{i \bullet}\left[\omega_{*}, y_{*}\right]\right) \frac{1}{\alpha s} \not p_{2} \not p\right. \\
& +\frac{i g}{2 \alpha} \int_{y_{*}}^{x_{*}} d \frac{2}{s} \omega_{*}\left(\left[x_{*}, \omega_{*}\right]\left(\frac{1}{2} \sigma^{i j} F_{i j}+\frac{4}{s^{2}} F_{\bullet *} \sigma_{* \bullet}\right)\left[\omega_{*}, y_{*}\right]\right. \\
& +\left[x_{*}, \omega_{*}\right] \frac{2}{s}\left(\omega_{*}-x_{*}\right)\left(i D^{i} F_{i}\left(\omega_{*}\right)\right)\left[\omega_{*}, y_{*}\right] \\
& -2 g \int_{\omega_{*}}^{x_{*}} d \frac{2}{s} \omega_{*}^{\prime}\left[x_{*}, \omega_{*}^{\prime}\right] \frac{2}{s}\left(\omega_{*}^{\prime}-x_{*}\right) F_{i \bullet}\left(\omega_{*}^{\prime}\right)\left[\omega_{*}^{\prime}, \omega_{*}\right] F^{i} \bullet\left(\omega_{*}\right)\left[\omega_{*}, y_{*}\right] \\
& \left.+2\left[x_{*}, \omega_{*}\right] \frac{2}{s}\left(\omega_{*}-x_{*}\right) F_{i \bullet}\left(\omega_{*}\right)\left[\omega_{*}, y_{*}\right] P^{i}\right) \frac{1}{\alpha s} \not p p_{2} \not p+\left[\frac{i g}{4 \alpha^{2}} \int_{y_{*}}^{x_{*}} d \frac{2}{s} \omega_{*}\left[x_{*}, \omega_{*}\right] \mathcal{B}_{1}\left(i D_{\perp}\left[\omega_{*}, y_{*}\right]\right)\right. \\
& +\frac{i g}{4 \alpha^{2}} \int_{y_{*}}^{x_{*}} d \frac{2}{s} \omega_{*}\left(i \mathscr{D}_{\perp}\left[x_{*}, \omega_{*}\right]\right) \mathcal{B}_{1}\left[\omega_{*}, y_{*}\right]+\frac{i g}{4 \alpha^{2}} \int_{y_{*}}^{x_{*}} d \frac{2}{s} \omega_{*} \frac{2}{s}\left(\omega_{*}-x_{*}\right) \\
& \times\left(-2\left[x_{*}, \omega_{*}\right]\left(i D_{i} \gamma^{j} F_{j \bullet}\right)\left[\omega_{*}, y_{*}\right] P^{i}-2\left[x_{*}, \omega_{*}\right]\left(i D^{i} \gamma^{j} F_{j \bullet}\right)\left(i \mathfrak{D}_{i}\left[\omega_{*}, y_{*}\right]\right)\right. \\
& +2\left(i \mathscr{D}_{\perp}\left[x_{*}, \omega_{*}\right]\right) F^{i} \bullet\left(i \mathfrak{D}_{i}\left[\omega_{*}, y_{*}\right]\right)+2\left[x_{*}, \omega_{*}\right] F_{i \bullet}\left(i \mathfrak{D}^{i}\left(i \mathscr{D}_{\perp}\left[\omega_{*}, y_{*}\right]\right)\right) \\
& +\left[x_{*}, \omega_{*}\right]\left(i D^{i} F_{i \bullet}\right)\left(i D_{\perp}\left[\omega_{*}, y_{*}\right]\right)+\left(i \mathscr{D}_{\perp}\left[x_{*}, \omega_{*}\right]\right)\left(i D^{i} F_{i \bullet}\right)\left[\omega_{*}, y_{*}\right] \\
& \left.\left.\left.+2\left[x_{*}, \omega_{*}\right] F_{i \bullet}\left(i \mathscr{D}_{\perp}\left[\omega_{*}, y_{*}\right]\right) P^{i}+2\left(i \mathscr{D}_{\perp}\left[x_{*}, \omega_{*}\right]\right) F_{i \bullet}\left[\omega_{*}, y_{*}\right] P^{i}\right)\right] \frac{2}{s} \not p_{2} \not p\right\} \\
& \times e^{i \frac{\hat{p}_{\perp}^{2}}{\alpha s}\left(y_{*}-x_{*}\right)}\left|y_{\perp}\right\rangle+O\left(\lambda^{-2}\right) .
\end{aligned}
$$


Equation (E.9) is the final result for the quark propagator with sub-eikonal corrections with point $x_{\perp}^{\mu}+\frac{2}{s} x_{*} p_{2}^{\mu}$ in the external field. Note that, in (E.9), the non gauge-invariant terms are those with gauge field $A_{i}$ only at point $y_{*}$ which, being outside the shock-wave, can be set to zero (see figure 5). In eq. (E.9) the action of the covariant derivative $\mathfrak{D}_{i}$ can be performed using its definition (A.3).

\section{F Gluon propagator in the background-Feynman gauge}

\section{F.1 In the background of gluon field}

Let us consider gluon propagator in the background-Feynman gauge $D^{\mu} A_{\mu}^{q}=0$ where again $D_{\mu}=\partial_{\mu}-i g A_{\mu}^{c l}$. The superscript "cl" will be omitted again from now on. The propagator in Schwinger formalism can be written as

$$
i\left\langle A_{\mu}^{a}(x) A_{\nu}^{b}(y)\right\rangle=\left\langle x\left|\frac{1}{\hat{P}^{2}+2 i g F+i \epsilon}\right| y\right\rangle_{\mu \nu}^{a b}
$$

where $\hat{P}^{2}=\hat{p}^{2}+2 g \alpha \hat{A}_{\bullet}+g\left\{\hat{p}_{\perp}^{\mu}, \hat{A}_{\mu}^{\perp}\right\}+\frac{2}{s} g\left\{\hat{P}_{\bullet}, \hat{A}_{*}\right\}-g^{2} \hat{A}_{\perp}^{2}$. We will omit the symbol ^ from the operators, for simplicity. We define again the operator $O \equiv\left\{p_{\perp}^{\mu}, A_{\mu}^{\perp}\right\}+\frac{2}{s}\left\{P_{\bullet}, A_{*}\right\}-g A_{\perp}^{2}$ and get rid of the term $\left\{P_{\bullet}, A_{*}\right\}$ as we did in section 3 .

Let us expand eq. (F.1) in $F_{\mu \nu}$ up to $F^{3}$ terms, which are the ones relevant to get the $\lambda^{-1}$ corrections (we will omit the $+i \epsilon$ prescription for each $\frac{1}{P^{2}}$ factor)

$$
\begin{aligned}
( & \left.\frac{1}{P^{2}+2 i g F+i \epsilon}\right)_{\mu \nu} \\
= & \frac{g_{\mu \nu}}{P^{2}}-2 i g \frac{1}{P^{2}} F_{\mu \nu} \frac{1}{P^{2}}-4 g^{2} \frac{1}{P^{2}} F_{\mu \xi} \frac{1}{P^{2}} F_{\nu}^{\xi} \frac{1}{P^{2}}+8 i g^{3} \frac{1}{P^{2}} F_{\mu \xi} \frac{1}{P^{2}} F^{\xi \eta} \frac{1}{P^{2}} F_{\eta \nu} \frac{1}{P^{2}}+\ldots \\
= & \frac{g_{\mu \nu}}{P^{2}}-\frac{4 i g}{s}\left(p_{2 \nu} g_{\mu \alpha}^{\perp}-p_{2 \mu} g_{\nu \alpha}^{\perp}\right) \frac{1}{P^{2}} F^{\alpha} \bullet \frac{1}{P^{2}}+\frac{16 g^{2} p_{2 \mu} p_{2 \nu}}{s^{2}} \frac{1}{P^{2}} F^{i} \bullet \frac{1}{P^{2}} F_{i} \bullet \frac{1}{P^{2}} \\
& +\left[-2 i g g_{\alpha \mu}^{\perp} g_{\beta \nu}^{\perp} \frac{1}{P^{2}} F^{\alpha \beta} \frac{1}{P^{2}}-\frac{8 i g}{s^{2}}\left(p_{1 \mu} p_{2 \nu}-p_{2 \mu} p_{1 \nu}\right) \frac{1}{P^{2}} F_{* \bullet} \frac{1}{P^{2}}\right. \\
& +\frac{8 g^{2} p_{2 \mu} g_{\alpha \nu}^{\perp}}{s} \frac{1}{P^{2}} F^{i} \bullet \frac{1}{P^{2}} F_{i}^{\alpha} \frac{1}{P^{2}}+\frac{8 g^{2} g_{\alpha \mu}^{\perp} p_{2 \nu}}{s} \frac{1}{P^{2}} F^{i \alpha} \frac{1}{P^{2}} F_{i} \bullet \frac{1}{P^{2}} \\
& +\frac{16 g^{2} p_{2 \mu} g_{\alpha \nu}^{\perp}}{s^{2}} \frac{1}{P^{2}} F_{\bullet *} \frac{1}{P^{2}} F^{\alpha} \cdot \frac{1}{P^{2}}+\frac{16 g^{2} p_{2 \nu} g_{\alpha \mu}^{\perp}}{s^{2}} \frac{1}{P^{2}} F^{\alpha} \bullet \frac{1}{P^{2}} F_{\bullet *} \frac{1}{P^{2}} \\
& -\frac{64 i g^{3} p_{2 \mu 2} p_{2 \nu}}{s^{3}} \frac{1}{P^{2}} F_{\bullet *} \frac{1}{P^{2}} F_{i \bullet} \frac{1}{P^{2}} F^{i} \bullet \frac{1}{P^{2}}+\frac{64 i g^{3} p_{2 \mu 2} p_{2 \nu}}{s^{3}} \frac{1}{P^{2}} F_{i \bullet} \frac{1}{P^{2}} F^{i} \bullet \frac{1}{P^{2}} F_{\bullet *} \frac{1}{P^{2}} \\
& \left.-\frac{32 i g^{3} p_{2 \mu 2} p_{2 \nu}}{s^{2}} \frac{1}{P^{2}} F_{i \bullet} \frac{1}{P^{2}} F^{i j} \frac{1}{P^{2}} F_{j \bullet} \frac{1}{P^{2}}\right]+O\left(\lambda^{-2}\right) .
\end{aligned}
$$

In eq. (F.2) the terms in the square bracket are sub-eikonal terms so, for those terms, for each of the $\frac{1}{P^{2}}$ factors, we need the leading-eikonal scalar propagator. The terms outside the square bracket, instead, contain both eikonal and sub-eikonal corrections so, for those terms, for each $\frac{1}{P^{2}}$, we need the scalar propagator with up to sub-eikonal corrections, eq. (3.7), in the adjoint representation. Therefore, neglecting the terms with fields at the 
edges of the gauge link, that is, at point $x_{*}$ and $y_{*}$, we arrive, after some algebra,

$$
\begin{aligned}
\left\langle A_{\mu}^{a}(x) A_{\nu}^{b}(y)\right\rangle_{A}= & {\left[-\int_{0}^{+\infty} \frac{d \alpha}{2 \alpha} \theta\left(x_{*}-y_{*}\right)+\int_{-\infty}^{0} \frac{d \alpha}{2 \alpha} \theta\left(y_{*}-x_{*}\right)\right] e^{-i \alpha\left(x_{\bullet}-y_{\bullet}\right)}\left\langle x_{\perp}\right| e^{-i \frac{\hat{p}_{\perp}^{2}}{\alpha s} x_{*}} } \\
& \times\left\{g_{\mu \nu}\left[x_{*}, y_{*}\right]^{a b}-\frac{2 g}{\alpha s} \int_{y_{*}}^{x_{*}} d \frac{2}{s} \omega_{*}\left[\left(p_{2 \nu} \delta_{\mu}^{j}-p_{2 \mu} \delta_{\nu}^{j}\right)\left[x_{*}, \omega_{*}\right] F_{j}\left[\omega_{*}, y_{*}\right]\right.\right. \\
& \left.+\frac{2 g}{\alpha s} p_{2 \mu} p_{2 \nu} \int_{\omega_{*}}^{x_{*}} d \frac{2}{s} \omega_{*}^{\prime}\left[x_{*}, \omega_{*}^{\prime}\right] F^{i} \bullet\left[\omega_{*}^{\prime}, \omega_{*}\right] F_{i \bullet}\left[\omega_{*}, y_{*}\right]\right]^{a b} \\
& +\mathfrak{B}_{1 \mu \nu}^{a b}\left(x_{*}, y_{*} ; p_{\perp}\right)+\mathfrak{B}_{2 \mu \nu}^{a b}\left(x_{*}, y_{*} ; p_{\perp}\right)+\mathfrak{B}_{3 \mu \nu}^{a b}\left(x_{*}, y_{*} ; p_{\perp}\right)+\mathfrak{B}_{4 \mu \nu}^{a b}\left(x_{*}, y_{*} ; p_{\perp}\right) \\
& \left.+\mathfrak{B}_{5 \mu \nu}^{a b}\left(x_{*}, y_{*} ; p_{\perp}\right)+\mathfrak{B}_{6 \mu \nu}^{a b}\left(x_{*}, y_{*} ; p_{\perp}\right)+O\left(\lambda^{-2}\right)\right\} e^{i \frac{p_{\perp}^{2}}{\alpha s} y_{*}}\left|y_{\perp}\right\rangle
\end{aligned}
$$

where we defined

$$
\begin{aligned}
& \mathfrak{B}_{1 \mu \nu}^{a b}\left(x_{*}, y_{*} ; p_{\perp}\right)=g_{\mu \nu} \frac{i g}{2 \alpha}\left[\int _ { y _ { * } } ^ { x _ { * } } d \frac { 2 } { s } \omega _ { * } \left(\left\{p^{i},\left[x_{*}, \omega_{*}\right] \frac{2}{s} \omega_{*} F_{i \bullet}\left(\omega_{*}\right)\left[\omega_{*}, y_{*}\right]\right\}\right.\right. \\
& \left.\left.+g \int_{\omega_{*}}^{x_{*}} d \frac{2}{s} \omega_{*}^{\prime} \frac{2}{s}\left(\omega_{*}-\omega_{*}^{\prime}\right)\left[x_{*}, \omega_{*}^{\prime}\right] F^{i} \cdot\left[\omega_{*}^{\prime}, \omega_{*}\right] F_{i \bullet}\left[\omega_{*}, y_{*}\right]\right)\right]^{a b} \\
& \mathfrak{B}_{2 \mu \nu}^{a b}\left(x_{*}, y_{*} ; p_{\perp}\right)=\frac{1}{\alpha}\left(\frac{2}{s} p_{2 \nu} \delta_{\mu}^{j}-\frac{2}{s} p_{2 \mu} \delta_{\nu}^{j}\right) \frac{i g}{2 \alpha} \int_{y_{*}}^{x_{*}} d \frac{2}{s} z_{1 *}\left\{\frac{2}{s} z_{1 *}\left\{p^{i},\left[x_{*}, z_{1 *}\right] i D_{i} F_{j \bullet}\left[z_{1 *}, y_{*}\right]\right\}\right. \\
& -g \int_{z_{1 *}}^{x_{*}} d \frac{2}{s} \omega_{*}\left[\frac{2}{s}\left(\omega_{*}-z_{1 *}\right)\left[x_{*}, \omega_{*}\right] i D_{i} F_{j \bullet}\left[\omega_{*}, z_{1 *}\right] F_{j \bullet}\left[z_{1 *}, y_{*}\right]\right. \\
& +\frac{2}{s}\left(\omega_{*}-z_{1 *}\right)\left[x_{*}, \omega_{*}\right] F^{i} \bullet\left[\omega_{*}, z_{1 *}\right] i D_{i} F_{j \bullet}\left[z_{1 *}, y_{*}\right] \\
& +\left\{p^{i},\left[x_{*}, \omega_{*}\right] \frac{2}{s} \omega_{*} F_{i \bullet}\left[\omega_{*}, z_{1 *}\right] F_{j \bullet}\left[z_{1 *}, y_{*}\right]\right\}+\left\{p^{i},\left[x_{*}, \omega_{*}\right] F_{j \bullet}\left[\omega_{*}, z_{1 *}\right] \frac{2}{s} z_{1 *} F_{i \bullet}\left[z_{1 *}, y_{*}\right]\right\} \\
& +g \int_{\omega_{*}}^{x_{*}} d \frac{2}{s} \omega_{*}^{\prime}\left(\frac{2}{s}\left(\omega_{*}-\omega_{*}^{\prime}\right)\left[x_{*}, \omega_{*}^{\prime}\right] F^{i} \bullet\left[\omega_{*}^{\prime}, \omega_{*}\right] F_{i \bullet}\left[\omega_{*}, z_{1 *}\right] F_{j \bullet}\left[z_{1 *}, y_{*}\right]\right. \\
& +\frac{2}{s}\left(z_{1 *}-\omega_{*}\right)\left[x_{*}, \omega_{*}^{\prime}\right] F_{j \bullet}\left[\omega_{*}^{\prime}, \omega_{*}\right] F^{i} \bullet\left[\omega_{*}, z_{1 *}\right] F_{i \bullet}\left[z_{1 *}, y_{*}\right] \\
& \left.\left.\left.+\frac{2}{s}\left(z_{1 *}-\omega_{*}^{\prime}\right)\left[x_{*}, \omega_{*}^{\prime}\right] F_{i \bullet}\left[\omega_{*}^{\prime}, \omega_{*}\right] F_{j \bullet}\left[\omega_{*}, z_{1 *}\right] F^{i} \cdot\left[z_{1 *}, y_{*}\right]\right)\right]\right\}^{a b} \\
& \mathfrak{B}_{3 \mu \nu}^{a b}\left(x_{*}, y_{*} ; p_{\perp}\right) \\
& =\frac{4 g p_{2 \mu} p_{2 \nu}}{\alpha^{2} s^{2}} \int_{y_{*}}^{x_{*}} d \frac{2}{s} z_{2 *}\left\{\frac { i g } { 2 \alpha } \int _ { z _ { 2 * } } ^ { x * } d \frac { 2 } { s } z _ { 1 * } \left[\frac{2}{s} z_{1 *}\left\{p^{i},\left[x_{*}, z_{1 *}\right] i D_{i} F^{j} \cdot\left[z_{1 *}, y_{*}\right] F_{j \bullet}\left[z_{2 *}, y_{*}\right]\right\}\right.\right. \\
& +\frac{2}{s} z_{2 *}\left\{p^{i},\left[x_{*}, z_{1 *}\right] F^{j} \bullet\left[z_{1 *}, z_{2 *}\right] i D_{i} F_{j \bullet}\left[z_{2 *}, y_{*}\right]\right\} \\
& +\frac{2}{s}\left(z_{1 *}-z_{2 *}\right)\left[x_{*}, z_{1 *}\right] i D_{i} F^{j} \bullet\left[z_{1 *}, z_{2 *}\right] i D^{i} F_{j \bullet}\left[z_{2 *}, y_{*}\right] \\
& -g \int_{z_{1 *}}^{x_{*}} d \frac{2}{s} \omega_{*}\left(\left\{p^{i},\left[x_{*}, \omega_{*}\right] \frac{2}{s} \omega_{*} F_{i \bullet}\left[\omega_{*}, z_{1 *}\right] F^{j} \bullet\left[z_{1 *}, z_{2 *}\right] F_{j \bullet}\left[z_{2 *}, y_{*}\right]\right\}\right.
\end{aligned}
$$




$$
\begin{aligned}
& +\left\{p^{i},\left[x_{*}, \omega_{*}\right] F^{j} \bullet\left[\omega_{*}, z_{1 *}\right] \frac{2}{s} z_{1 *} F_{i \bullet}\left[z_{1 *}, z_{2 *}\right] F_{j \bullet}\left[z_{2 *}, y_{*}\right]\right\} \\
& +\left\{p^{i},\left[x_{*}, \omega_{*}\right] F^{j} \cdot\left[\omega_{*}, z_{1 *}\right] F_{j \bullet[}\left[z_{1 *}, z_{2 *}\right] \frac{2}{s} z_{2 *} F_{i \bullet}\left[z_{2 *}, y_{*}\right]\right\} \\
& +\frac{2}{s}\left(\omega_{*}-z_{2 *}\right)\left[x_{*}, \omega_{*}\right] F^{i} \bullet\left[\omega_{*}, z_{1 *}\right] F^{j} \cdot\left[z_{1 *}, z_{2 *}\right] i D_{i} F_{j \bullet}\left[z_{2 *}, y_{*}\right] \\
& +\frac{2}{s}\left(z_{1 *}-z_{2 *}\right)\left[x_{*}, \omega_{*}\right] F^{j} \cdot\left[\omega_{*}, z_{1 *}\right] F^{i} \cdot\left[z_{1 *}, z_{2 *}\right] i D_{i} F_{j \bullet[}\left[z_{2 *}, y_{*}\right] \\
& +\frac{2}{s}\left(z_{1 *}-z_{2 *}\right)\left[x_{*}, \omega_{*}\right] F^{j} \cdot\left[\omega_{*}, z_{1 *}\right] i D_{i} F_{j \bullet}\left[z_{1 *}, z_{2 *}\right] F^{i} \bullet\left[z_{2 *}, y_{*}\right] \\
& +\frac{2}{s}\left(\omega_{*}-z_{2 *}\right)\left[x_{*}, \omega_{*}\right] i D_{i} F^{j} \cdot\left[\omega_{*}, z_{1 *}\right] F_{j \bullet}\left[z_{1 *}, z_{2 *}\right] F^{i} \bullet\left[z_{2 *}, y_{*}\right] \\
& +\frac{2}{s}\left(\omega_{*}-z_{1 *}\right)\left[x_{*}, \omega_{*}\right] i D_{i} F^{j} \bullet\left[\omega_{*}, z_{1 *}\right] F^{i} \bullet\left[z_{1 *}, z_{2 *}\right] F_{j \bullet}\left[z_{2 *}, y_{*}\right] \\
& +\frac{2}{s}\left(\omega_{*}-z_{1 *}\right)\left[x_{*}, \omega_{*}\right] F^{i} \bullet\left[\omega_{*}, z_{1 *}\right] i D_{i} F^{j} \bullet\left[z_{1 *}, z_{2 *}\right] F_{j \bullet}\left[z_{2 *}, y_{*}\right] \\
& +2 i \frac{2}{s}\left[x_{*}, \omega_{*}\right] F_{\bullet *}\left[\omega_{*}, z_{1 *}\right] F_{i \bullet}\left[z_{1 *}, z_{2 *}\right] F_{\bullet}^{i}\left[z_{2 *}, y_{*}\right] \\
& -2 i \frac{2}{s}\left[x_{*}, \omega_{*}\right] F^{i} \bullet\left[\omega_{*}, z_{1 *}\right] F_{i \bullet}\left[z_{1 *}, z_{2 *}\right] F_{\bullet *}\left[z_{2 *}, y_{*}\right] \\
& +2 i\left[x_{*}, \omega_{*}\right] F^{i} \cdot\left[\omega_{*}, z_{1 *}\right] F_{i j}\left[z_{1 *}, z_{2 *}\right] F^{j} \cdot\left[z_{2 *}, y_{*}\right] \\
& +g \int_{\omega_{*}}^{x_{*}} d \frac{2}{s} \omega_{*}^{\prime}\left(\frac{2}{s}\left(z_{2 *}-z_{1 *}+\omega_{*}-\omega_{*}^{\prime}\right)\left[x_{*}, \omega_{*}^{\prime}\right] F^{i} \cdot\left[\omega_{*}^{\prime}, \omega_{*}\right] F_{i \bullet}\left[\omega_{*}, z_{1 *}\right] F^{j} \bullet\left[z_{1 *}, z_{2 *}\right] F_{j \bullet[}\left[z_{2 *}, y_{*}\right]\right. \\
& +\frac{2}{s}\left(z_{2 *}+z_{1 *}-\omega_{*}-\omega_{*}^{\prime}\right)\left[x_{*}, \omega_{*}^{\prime}\right] F^{j} \cdot\left[\omega_{*}^{\prime}, \omega_{*}\right] F^{i} \bullet\left[\omega_{*}, z_{1 *}\right] F_{i \bullet}\left[z_{1 *}, z_{2 *}\right] F_{j \bullet}\left[z_{2 *}, y_{*}\right] \\
& \left.\left.\left.\left.+\frac{2}{s}\left(z_{2 *}+z_{1 *}-\omega_{*}-\omega_{*}^{\prime}\right)\left[x_{*}, \omega_{*}^{\prime}\right] F_{i \bullet}\left[\omega_{*}^{\prime}, \omega_{*}\right] F^{j} \cdot\left[\omega_{*}, z_{1 *}\right] F^{i} \cdot\left[z_{1 *}, z_{2 *}\right] F_{j \bullet}\left[z_{2 *}, y_{*}\right]\right)\right)\right]\right\}^{a b}
\end{aligned}
$$

$$
\begin{aligned}
\mathfrak{B}_{4 \mu \nu}^{a b}\left(x_{*}, y_{*} ; p_{\perp}\right)= & -\frac{2 g^{2}}{\alpha^{2} s} \int_{y_{*}}^{x_{*}} d \frac{2}{s} \omega_{*}\left[p_{2 \mu} \delta_{\nu}^{j} \int_{\omega_{*}}^{x_{*}} d \frac{2}{s} \omega_{*}^{\prime}\left[x_{*}, \omega_{*}^{\prime}\right] F_{\bullet}^{i}\left[\omega_{*}^{\prime}, \omega_{*}\right] F_{i j}\left[\omega_{*}, y_{*}\right]\right. \\
& +p_{2 \nu} \delta_{\mu}^{j} \int_{\omega_{*}}^{x} d \frac{2}{s} \omega_{*}^{\prime}\left[x_{*}, \omega_{*}^{\prime}\right] F_{i j}\left[\omega_{*}^{\prime}, \omega_{*}\right] F_{\bullet}^{i}\left[\omega_{*}, y_{*}\right] \\
& +p_{2 \mu} \delta_{\nu}^{j} \int_{\omega_{*}}^{x} d \frac{2}{s} \omega_{*}^{\prime}\left[x_{*}, \omega_{*}^{\prime}\right] \frac{2}{s} F_{\bullet *}\left[\omega_{*}^{\prime}, \omega_{*}\right] F_{j \bullet}\left[\omega_{*}, y_{*}\right] \\
& \left.+p_{2 \nu} \delta_{\mu}^{j} \int_{\omega_{*}}^{x_{*}} d \frac{2}{s} \omega_{*}^{\prime}\left[x_{*}, \omega_{*}^{\prime}\right] F_{j \bullet}\left[\omega_{*}^{\prime}, \omega_{*}\right] \frac{2}{s} F_{\bullet *}\left[\omega_{*}, y_{*}\right]\right]^{a b}
\end{aligned}
$$$$
\mathfrak{B}_{5 \mu \nu}^{a b}\left(x_{*}, y_{*} ; p_{\perp}\right)=-\frac{g}{\alpha} \delta_{\mu}^{i} \delta_{\nu}^{j} \int_{y_{*}}^{x_{*}} d \frac{2}{s} \omega_{*}\left(\left[x_{*}, \omega_{*}\right] F_{i j}\left[\omega_{*}, y_{*}\right]\right)^{a b}
$$$$
\mathfrak{B}_{6 \mu \nu}^{a b}\left(x_{*}, y_{*} ; p_{\perp}\right)=-\frac{4 g}{\alpha s^{2}}\left(p_{1 \mu} p_{2 \nu}-p_{2 \mu} p_{1 \nu}\right) \int_{y_{*}}^{x_{*}} d \frac{2}{s} \omega_{*}\left(\left[x_{*}, \omega_{*}\right] F_{* \bullet}\left[\omega_{*}, y_{*}\right]\right)^{a b}
$$

Equation (F.3) is the final result of the gluon propagator with sub-eikonal corrections in the background-Feynman gauge. The operators $\mathfrak{B}_{1 \mu \nu}^{a b}, \mathfrak{B}_{2 \mu \nu}^{a b}, \mathfrak{B}_{3 \mu \nu}^{a b}, \mathfrak{B}_{4 \mu \nu}^{a b}, \mathfrak{B}_{5 \mu \nu}^{a b}$, and $\mathfrak{B}_{6 \mu \nu}^{a b}$ are the sub-eikonal corrections to the gluon propagator in the gluon external filed in the background-Feynman gauge. 


\section{F.2 In the background of quark and anti-quark fields}

Let us consider the gluon propagator in the background of quark and anti-quark fields. As we have anticipated in 5.2, in the background-Feynman gauge the gluon propagator in the background of quark and anti quark fields receives a eikonal contribution. The diagram and the cross-diagram is given in figure 3

$$
\begin{aligned}
\left\langle A_{\mu}^{a}(x) A_{\nu}^{b}(y)\right\rangle_{\psi \bar{\psi}}= & -g^{2} \int d^{4} z_{1} d^{4} z_{2}\left\langle A_{\mu}^{a}(x) A_{\rho}^{c}\left(z_{1}\right)\right\rangle\left\langle A_{\lambda}^{d}\left(z_{2}\right) A_{\nu}^{b}(y)\right\rangle \\
& \times\left[\bar{\psi}\left(z_{2}\right) t^{d} \gamma^{\lambda}\left\langle z_{2}\left|\frac{i}{P+i \epsilon}\right| z_{1}\right\rangle \gamma^{\rho} t^{c} \psi\left(z_{1}\right)+\bar{\psi}\left(z_{1}\right) t^{c} \gamma^{\rho}\left\langle z_{1}\left|\frac{i}{P+i \epsilon}\right| z_{2}\right\rangle \gamma^{\lambda} t^{d} \psi\left(z_{2}\right)\right]
\end{aligned}
$$

To proceed, we need the eikonal gluon propagator in the gluon external field with the transverse $A_{i}$ fields at the edge of the gauge link (i.e. at point $z_{1 *}$ and $z_{2 *}$ ) not set to zero like eq. (3.7) for the scalar propagator. The reason is two folds. First, we need to extract eikonal and sub-eikonal contributions and second, the gluon propagator has points $z_{1 *}$ and $z_{2 *}$ in the shock-wave external field. Thus, we have

$$
\begin{aligned}
\left\langle A_{\mu}^{a}(x) A_{\rho}^{c}\left(z_{1}\right)\right\rangle_{A}= & {\left[-\int_{0}^{+\infty} \frac{d \alpha}{2 \alpha} \theta\left(x_{*}-z_{1 *}\right)+\int_{-\infty}^{0} \frac{d \alpha}{2 \alpha} \theta\left(z_{1 *}-x_{*}\right)\right] e^{-i \alpha\left(x_{\bullet}-z_{1}\right)}\left\langle x_{\perp}\right| e^{-i \frac{\hat{p}_{\perp}^{2}}{\alpha s} x_{*}} } \\
& \times\left\{g_{\mu \rho}\left[x_{*}, z_{1 *}\right]^{a c}-g_{\mu \rho} \frac{i g}{2 \alpha}\left[x_{*}, z_{1 *}\right]^{a c} \frac{2}{s} z_{1 *}\left(\left\{P_{i}, A^{i}\left(z_{1 *}\right)\right\}-g A_{i}\left(z_{1 *}\right) A^{i}\left(z_{1 *}\right)\right)\right. \\
& -\frac{2 g}{\alpha s} \int_{z_{1 *}}^{x_{*}} d \frac{2}{s} \omega_{*}\left(\left(p_{2 \rho} \delta_{\mu}^{j}-p_{2 \mu} \delta_{\rho}^{j}\right)\left[x_{*}, \omega_{*}\right] F_{j \bullet}\left[\omega_{*}, z_{1 *}\right]\right. \\
& \left.\left.+\frac{2 g}{\alpha s} p_{2 \mu} p_{2 \rho} \int_{\omega_{*}}^{x_{*}} d \frac{2}{s} \omega_{*}^{\prime}\left[x_{*}, \omega^{\prime}\right] F_{\bullet}^{i}\left[\omega_{*}^{\prime}, \omega_{*}\right] F_{\bullet}\left[\omega_{*}, z_{1 *}\right]\right)^{a c}\right\} e^{i \frac{\hat{p}_{\perp}^{2}}{\alpha s} z_{1 *}\left|z_{1 \perp}\right\rangle}
\end{aligned}
$$

and

$$
\begin{aligned}
\left\langle A_{\lambda}^{d}\left(z_{2}\right) A_{\nu}^{b}(y)\right\rangle_{A}= & {\left[-\int_{0}^{+\infty} \frac{d \alpha}{2 \alpha} \theta\left(z_{2 *}-y_{*}\right)+\int_{-\infty}^{0} \frac{d \alpha}{2 \alpha} \theta\left(y_{*}-z_{2 *}\right)\right] e^{-i \alpha\left(z_{2}-y_{\bullet}\right)}\left\langle z_{2 \perp}\right| e^{-i \frac{\hat{p}_{\perp}^{2}}{\alpha s} z_{2 *}} } \\
& \times\left\{g_{\lambda \nu}\left[z_{2 *}, y_{*}\right]^{d b}+g_{\lambda \nu} \frac{i g}{2 \alpha} \frac{2}{s} z_{2 *}\left(\left\{P_{i}, A^{i}\left(z_{2 *}\right)\right\}-g A_{i}\left(z_{2 *}\right) A^{i}\left(z_{2 *}\right)\right)\left[z_{2 *}, y_{*}\right]^{d b}\right. \\
& -\frac{2 g}{\alpha s} \int_{y_{*}}^{z_{2 *}} d \frac{2}{s} \omega_{*}\left(\left(p_{2 \nu} \delta_{\lambda}^{j}-p_{2 \lambda} \delta_{\nu}^{j}\right)\left[z_{2 *}, \omega_{*}\right] F_{j \bullet}\left[\omega_{*}, y_{*}\right]\right. \\
& \left.\left.+\frac{2 g}{\alpha s} p_{2 \lambda} p_{2 \nu} \int_{\omega_{*}}^{z_{2 *}} d \frac{2}{s} \omega_{*}^{\prime}\left[z_{2 *}, \omega_{*}^{\prime}\right] F_{\bullet}^{i}\left[\omega_{*}^{\prime}, \omega_{*}\right] F_{i \bullet}\left[\omega_{*}, y_{*}\right]\right)^{d b}\right\} e^{i \frac{\hat{p}_{\perp}^{2}}{\alpha s} y_{*}\left|y_{\perp}\right\rangle .}
\end{aligned}
$$


It is convenient to start with a symmetric expression with respect to $\not P$

$$
\begin{aligned}
\bar{\psi} & \left(z_{1}\right) t^{c} \gamma^{\rho}\left(\frac{1}{2}\left\langle z_{1}\left|\not P \frac{i}{P^{2}+i \epsilon}\right| z_{2}\right\rangle+\frac{1}{2}\left\langle z_{1}\left|\frac{i}{P^{2}+i \epsilon} \not P\right| z_{2}\right\rangle\right) \gamma^{\lambda} t^{d} \psi\left(z_{2}\right) \\
= & \left\langle z_{1}\right|\left(\frac{8}{s^{2}} p_{2}^{\rho} p_{2}^{\lambda} \bar{\psi} t^{c} \not p_{1} \frac{1}{2}\left\{P \bullet \frac{i}{P^{2}+i \epsilon}\right\} t^{d} \psi+\bar{\psi} t^{c} \not p_{1} \frac{1}{2}\left(\frac{2}{s} p_{2}^{\rho} \gamma^{j} \gamma_{\perp}^{\lambda}+\frac{2}{s} p_{2}^{\lambda} \gamma_{\perp}^{\rho} \gamma^{j}\right)\left\{P_{j}, \frac{i}{P^{2}+i \epsilon}\right\} t^{d} \psi\right. \\
& +\frac{2}{s} \bar{\psi} t^{c} \gamma_{\perp}^{\rho} P_{*} \not p_{1} \gamma_{\perp}^{\lambda} \frac{i}{P^{2}+i \epsilon} t^{d} \psi-\frac{8}{s^{2}} p_{2}^{\rho} p_{2}^{\lambda} \bar{\psi} t^{c} \not p_{1} \frac{1}{2}\left[\not P_{\perp}, \frac{i}{P^{2}+i \epsilon} g F_{i} \bullet \gamma^{i} \frac{i}{P^{2}+i \epsilon}\right] t^{d} \psi \\
& \left.+\frac{8}{s^{2}} \bar{\psi} t^{c} \frac{1}{2}\left(p_{2}^{\lambda} \gamma_{\perp}^{\rho} \gamma^{i}-p_{2}^{\rho} \gamma^{i} \gamma_{\perp}^{\lambda}\right) \not p_{1} P_{*} \frac{i}{P^{2}+i \epsilon} g F_{i \bullet} \frac{i}{P^{2}+i \epsilon} t^{d} \psi\right)\left|z_{2}\right\rangle .
\end{aligned}
$$

Note that, we can use $P_{*}=p_{*}=\frac{s}{2} \alpha$ because $A_{*}$ will contribute as a sub-sub-eikonal term. In eq. (F.10) we have two integrations over the $*$-components $z_{1 *}$ and $z_{2 *}$, so we can use $p^{2}=p^{2}+2 \alpha g A_{\bullet}+i g \frac{2}{s} F_{i} \bullet \gamma^{i} p_{2}+O\left(\lambda^{-1}\right)$ neglecting all other sub-eikonal terms because they would contribute as sub-sub-eikonal corrections.

The term from which we will extract both eikonal and sub-eikonal contributions is $\frac{8}{s^{2}} p_{2}^{\rho} p_{2}^{\lambda} \bar{\psi} t^{c} \not p_{1} P \bullet \frac{i}{P^{2}} t^{d} \psi$. Let us observe that, using scalar propagator (3.7), we have

$$
\begin{aligned}
& \left\langle z_{1}\left|P \bullet \frac{i}{p^{2}+2 \alpha g A \bullet+g O}\right| z_{2}\right\rangle=i D_{\bullet}^{z_{1}}\left\langle z_{1}\left|\frac{i}{p^{2}+2 \alpha g A \bullet+g O}\right| z_{2}\right\rangle \\
& =\left\langle z_{1}\left|\frac{i}{2 \alpha}\right| z_{2}\right\rangle+\left[\int_{0}^{+\infty} \frac{d \alpha}{2 \alpha} \theta\left(z_{1 *}-z_{2 *}\right)-\int_{-\infty}^{0} \frac{d \alpha}{2 \alpha} \theta\left(z_{2 *}-z_{1 *}\right)\right] e^{-i \alpha\left(z_{1} \bullet-z_{2} \bullet\right.}\left[\left\langle z_{1 \perp}\right| e^{-i \frac{\hat{p}_{\perp}^{2}}{\alpha s} z_{1 *}}\right. \\
& \quad \times\left\{\frac{\hat{p}_{\perp}^{2}}{2 \alpha}\left[z_{1 *}, z_{2 *}\right]-\frac{g}{2 \alpha}\left(\left\{P_{i}, A^{i}\left(z_{1 *}\right)\right\}-g A_{i}\left(z_{1 *}\right) A^{i}\left(z_{1 *}\right)\right)\left[z_{1 *}, z_{2 *}\right]\right\} e^{i \frac{\hat{p}_{\perp}^{2}}{\alpha s} z_{2 *}}\left|z_{2 \perp}\right\rangle \\
& \left.=\left\langle z_{1}\left|\frac{i}{2 \alpha}\right| z_{2}\right\rangle+\left[\int_{0}^{+\infty} \frac{d \alpha}{2 \alpha} \theta\left(z_{1 *}-z_{2 *}\right)-\int_{-\infty}^{0} \frac{d \alpha}{2 \alpha} \theta\left(z_{2 *}-z_{1 *}\right)\right] e^{-i \alpha\left(z_{1} \bullet-z_{2} \bullet\right.}\right) \\
& \quad \times\left\langle z_{1 \perp}\left|e^{-i \frac{\hat{p}_{\perp}^{2}}{\alpha s} z_{1 *}} P_{\perp}^{2}\left[z_{1 *}, z_{2 *}\right] e^{i \frac{\hat{p}_{\perp}^{2}}{\alpha s} z_{2 *}}\right| z_{2 \perp}\right\rangle
\end{aligned}
$$

and similarly we have

$$
\begin{aligned}
& \left\langle z_{1}\left|\frac{i}{p^{2}+2 \alpha g A_{\bullet}+g O} P \bullet\right| z_{2}\right\rangle \\
& \left.=\left\langle z_{1}\left|\frac{i}{2 \alpha}\right| z_{2}\right\rangle+\left[\int_{0}^{+\infty} \frac{d \alpha}{2 \alpha} \theta\left(z_{1 *}-z_{2 *}\right)-\int_{-\infty}^{0} \frac{d \alpha}{2 \alpha} \theta\left(z_{2 *}-z_{1 *}\right)\right] e^{-i \alpha\left(z_{1} \bullet-z_{2} \bullet\right.}\right) \\
& \quad \times\left\langle z_{1 \perp}\left|e^{-i \frac{\hat{p}_{\perp}^{2}}{\alpha s} z_{1 *}}\left[z_{1 *}, z_{2 *}\right] P_{\perp}^{2} e^{i \frac{\hat{p}_{\perp}^{2}}{\alpha s} z_{2 *}}\right| z_{2 \perp}\right\rangle
\end{aligned}
$$

where $P_{\perp}^{2}=-P^{i} P_{i}$, and in section 3 we defined $O=\left\{p_{\mu}, A_{\perp}^{\mu}\right\}-g A_{\perp}^{2}$.

Let us consider first the term $\left\langle z_{1}\left|\frac{i}{2 \alpha}\right| z_{2}\right\rangle$ from which we will have to extract eikonal and sub-eikonal corrections. Adding also the cross diagram, we have

$$
\begin{aligned}
\left\langle A_{\mu}^{a}(x) A_{\nu}^{b}(y)\right\rangle_{\psi \bar{\psi}} \ni & -g^{2} \int d^{4} z_{1} d^{4} z_{2}\left\langle A_{\mu}^{a}(x) A_{\rho}^{c}\left(z_{1}\right)\right\rangle\left\langle A_{\lambda}^{d}\left(z_{2}\right) A_{\nu}^{b}(y)\right\rangle \frac{8}{s^{2}} p_{2}^{\rho} p_{2}^{\lambda} \\
& \times\left[\bar{\psi}\left(z_{2}\right) \not p_{1} t^{d}\left\langle z_{1}\left|\frac{i}{2 \alpha}\right| z_{2}\right\rangle t^{c} \psi\left(z_{1}\right)+\bar{\psi}\left(z_{1}\right) \not p_{1} t^{c}\left\langle z_{1}\left|\frac{i}{2 \alpha}\right| z_{2}\right\rangle t^{d} \psi\left(z_{2}\right)\right] .
\end{aligned}
$$


Using propagators (F.11) and (F.12), and the identity $g \bar{\psi} \not p_{1}\left[t^{c}, t^{d}\right] \psi=\left(D^{i} F_{i \bullet}\right)^{c d}+\frac{2}{s}\left(D \bullet F_{* \bullet}\right)^{c d}$, we obtain

$$
\begin{aligned}
\left\langle A_{\mu}^{a}(x) A_{\nu}^{b}(y)\right\rangle_{\psi \bar{\psi}} \ni & -g^{2} \int d^{4} z_{1} d^{4} z_{2}\left\langle A_{\mu}^{a}(x) A_{\rho}^{c}\left(z_{1}\right)\right\rangle\left\langle A_{\lambda}^{d}\left(z_{2}\right) A_{\nu}^{b}(y)\right\rangle \frac{8}{s^{2}} p_{2}^{\rho} p_{2}^{\lambda} \\
& \times\left[\bar{\psi}\left(z_{2}\right) \not p_{1} t^{d}\left\langle z_{1}\left|\frac{i}{2 \alpha}\right| z_{2}\right\rangle t^{c} \psi\left(z_{1}\right)+\bar{\psi}\left(z_{1}\right) \not p_{1} t^{c}\left\langle z_{1}\left|\frac{i}{2 \alpha}\right| z_{2}\right\rangle t^{d} \psi\left(z_{2}\right)\right] \\
= & \left.-\int_{0}^{+\infty} \frac{d \alpha}{8 \alpha^{3}} \theta\left(x_{*}-y_{*}\right)+\int_{-\infty}^{0} \frac{d \alpha}{8 \alpha^{3}} \theta\left(y_{*}-x_{*}\right)\right] e^{-i \alpha\left(x_{\bullet}-y_{\bullet}\right)} \\
& \times \frac{8}{s^{2}} p_{2 \mu} p_{2 \nu}\left\langle x_{\perp}\right| e^{-i \frac{\hat{p}_{\perp}^{2}}{\alpha s} x_{*}} i g \int_{y_{*}}^{x_{*}} d \frac{2}{s} z_{1 *}\left[x_{*}, z_{1 *}\right]^{a c}\left(\left(D^{i} F_{i \bullet}\right)^{c d}+\frac{2}{s}\left(D_{\bullet} F_{* \bullet}\right)^{c d}\right. \\
& \left.-\frac{i z_{1 *}}{\alpha s}\left[\left\{P^{k},\left(i D_{k} D^{i} F_{i \bullet}\right)+\frac{2}{s}\left(i D_{k} D \bullet F_{*}\right)\right\}\right]^{c d}\right)\left[z_{1 *}, y_{*}\right]^{d b} e^{i \frac{\hat{p}_{\perp}^{2}}{\alpha s} y_{*}}\left|y_{\perp}\right\rangle .
\end{aligned}
$$

To arrive at result (F.17) we have used $e^{-i \frac{\hat{p}_{\perp}^{2}}{\alpha s} z_{*}} \psi\left(z_{*}\right) e^{i \frac{\hat{p}_{\perp}^{2}}{\alpha s} z_{*}}=\psi\left(z_{*}\right)+O(\lambda)$ and similarly for $\bar{\psi}$. The terms $\left(D^{i} F_{i \bullet}\right)$ and $\frac{2}{s}\left(D_{\bullet} F_{* \bullet}\right)^{c d}$ in eq. (F.17) are the eikonal contributions to the gluon propagator in the background of quark and anti-quark fields, while $\left\{P^{k},\left(i D_{k} D^{i} F_{i \bullet}\right)+\right.$ $\left.\frac{2}{s}\left(i D_{k} D_{\bullet} F_{* \bullet}\right)\right\}$ is one of the sub-eikonal terms. Note that to arrive at eq. (F.17) we made use of

$$
\begin{aligned}
e^{i \frac{\hat{p}_{\perp}^{2}}{\alpha s} z_{1 *}}\left(\left(D^{i} F_{i \bullet}\right)+\frac{2}{s}\left(D \bullet F_{* \bullet}\right)\right) e^{-i \frac{\hat{p}_{\perp}^{2}}{\alpha s} z_{1 *}} \\
=\left(D^{i} F_{i \bullet}\right)+\frac{2}{s}\left(D \bullet F_{* \bullet \bullet}\right)+\frac{i z_{1 *}}{\alpha s}\left[\hat{p}_{\perp}^{2},\left(D^{i} F_{i \bullet}\right)+\frac{2}{s}\left(D \bullet F_{* \bullet}\right)\right]+O\left(\lambda^{-2}\right) \\
=\left(D^{i} F_{i \bullet}\right)+\frac{2}{s}\left(D \cdot F_{* \bullet}\right)-\frac{i z_{1 *}}{\alpha s}\left[g\left(\left\{P^{j}, A_{j}\right\}-g A^{j} A_{j}\right)\left(\left(D^{i} F_{i \bullet}\right)+\frac{2}{s}\left(D_{\bullet} F_{* \bullet}\right)\right)\right. \\
\left.-g\left(\left(D^{i} F_{i \bullet}\right)+\frac{2}{s}\left(D \bullet F_{* \bullet}\right)\right)\left(\left\{P^{j}, A_{j}\right\}-g A^{j} A_{j}\right)-\left\{P^{j},\left(i D_{j}\left(D^{i} F_{i \bullet}\right)\right)+\frac{2}{s}\left(i D_{j} D \bullet F_{* \bullet}\right)\right\}\right]+O\left(\lambda^{-2}\right) .
\end{aligned}
$$

where $g\left(\left\{P^{j}, A_{j}\right\}-g A^{j} A_{j}\right)\left(\left(D^{i} F_{i \bullet}\right)+\frac{2}{s}\left(D \bullet F_{* \bullet}\right)\right)$ and $-g\left(\left(D^{i} F_{i \bullet}\right)+\frac{2}{s}\left(D_{\bullet} F_{* \bullet}\right)\right)\left(\left\{P^{j}, A_{j}\right\}-\right.$ $\left.g A^{j} A_{j}\right)$ will cancel out with similar terms coming from the gluon propagators (F.11) and (F.12). We can rewrite eq. (F.17), pushing the operator $P^{j}$ all to the left and to the right of the gauge links, as

$$
\begin{aligned}
& \left\langle A_{\mu}^{a}(x) A_{\nu}^{b}(y)\right\rangle_{\psi \bar{\psi}} \ni\left[-\int_{0}^{+\infty} \frac{d \alpha}{8 \alpha^{3}} \theta\left(x_{*}-y_{*}\right)+\int_{-\infty}^{0} \frac{d \alpha}{8 \alpha^{3}} \theta\left(y_{*}-x_{*}\right)\right] e^{-i \alpha\left(x_{\bullet}-y_{\bullet}\right)} \\
& \quad \times \frac{8}{s^{2}} p_{2 \mu} p_{2 \nu}\left\langle x_{\perp}\right| e^{-i \frac{\hat{p}_{\perp}^{2}}{\alpha s} x_{*}} i g \int_{y_{*}}^{x_{*}} d \frac{2}{s} z_{1 *}\left[\left[x_{*}, z_{1 *}\right]^{a c}\left(\left(D^{i} F_{\boldsymbol{}_{\bullet}}\right)+\frac{2}{s}\left(D_{\bullet} F_{* \bullet}\right)\right)^{c d}\left[z_{1 *}, y_{*}\right]^{d b}\right. \\
& \quad+\frac{z_{1 *}}{\alpha s}\left\{P^{k},\left[x_{*}, z_{1 *}\right]^{a c}\left(\left(D_{k} D^{i} F_{\bullet}\right)+\frac{2}{s}\left(D_{k} D \bullet F_{*}\right)\right)^{c d}\left[z_{1 *}, y_{*}\right]^{d b}\right\} \\
& \quad+\left(\frac{z_{1 *}}{\alpha s}\right) g \int_{z_{1 *}}^{x_{*}} d \frac{2}{s} \omega_{*}\left(\left(\left[x_{*}, \omega_{*}\right] F_{k} \bullet\left[\omega_{*}, z_{1 *}\right]\right)^{a c}\left(\left(D^{k} D^{i} F_{\bullet \bullet}\right)+\frac{2}{s}\left(D^{k} D \cdot F_{* \bullet}\right)\right)^{c d}\left[z_{1 *}, y_{*}\right]^{d b}\right. \\
& \left.\left.\quad-\left[x_{*}, \omega_{*}\right]^{a c}\left(\left(D^{k} D^{i} F_{i \bullet}\right)+\frac{2}{s}\left(D^{k} D_{\bullet} F_{* \bullet}\right)\right)^{c d}\left(\left[\omega_{*}, z_{1 *}\right] F_{k \bullet}\left[z_{1 *}, y_{*}\right]\right)^{d b}\right)\right] e^{i \frac{\hat{p}_{\perp}^{2}}{\alpha s} y_{*}}\left|y_{\perp}\right\rangle .
\end{aligned}
$$

Note that, to get eq. (F.19) we have also added the cross diagram. 
Next, we consider the terms $P_{\perp}^{2}\left[z_{1 *}, z_{2 *}\right]$ and $\left[z_{1 *}, z_{2 *}\right] P_{\perp}^{2}$ (without the cross diagram) which are sub-eikonal corrections. We have

$$
\begin{aligned}
& \left\langle A_{\mu}^{a}(x) A_{\nu}^{b}(y)\right\rangle_{\psi \bar{\psi}} \ni g^{2} \int d^{4} z_{1} d^{4} z_{2}\left\langle A_{\mu}^{a}(x) A_{\rho}^{c}\left(z_{1}\right)\right\rangle\left\langle A_{\lambda}^{d}\left(z_{2}\right) A_{\nu}^{b}(y)\right\rangle \\
& \quad \times\left[-\int_{0}^{+\infty} \frac{d \alpha}{2 \alpha} \theta\left(z_{1 *}-z_{2 *}\right)+\int_{-\infty}^{0} \frac{d \alpha}{2 \alpha} \theta\left(z_{2 *}-z_{1 *}\right)\right] e^{-i \alpha\left(z_{1} \bullet-z_{2} \bullet\right.} \frac{4}{s^{2}} p_{2 \mu} p_{2 \nu} \\
& \quad \times \bar{\psi}\left(z_{1}\right) t^{c} p_{1}\left\langle z_{1 \perp}\right| e^{-i \frac{\hat{p}_{\perp}^{2}}{\alpha s} z_{1 *}} \frac{1}{2 \alpha}\left(P_{\perp}^{2}\left[z_{1 *}, z_{2 *}\right]+\left[z_{1 *}, z_{2 *}\right] P_{\perp}^{2}\right) e^{i \frac{\hat{p}_{\perp}^{2}}{\alpha s} z_{2 *}\left|z_{2 \perp}\right\rangle t^{d} \psi\left(z_{2}\right)} \\
& =\left[-\int_{0}^{+\infty} \frac{d \alpha}{2 \alpha} \theta\left(x_{*}-y_{*}\right)+\int_{-\infty}^{0} \frac{d \alpha}{2 \alpha} \theta\left(y_{*}-x_{*}\right)\right] e^{-i \alpha\left(x_{\bullet}-y_{\bullet}\right)}\left\langle x_{\perp}\right| e^{-i \frac{\hat{p}_{\perp}^{2}}{\alpha s} x_{*}} \\
& \quad \times \frac{g^{2}}{8 \alpha^{3}} \frac{4}{s^{2}} p_{2 \mu} p_{2 \nu} \int_{x_{*}}^{y_{*}} d \frac{2}{s} z_{1 *} \int_{y_{*}}^{z_{1 *}} d \frac{2}{s} z_{2 *}\left(\left[P_{\perp}^{2} \bar{\psi}\left(z_{1 *}\right)-\left(\bar{\psi}\left(z_{1 *}\right) \overleftarrow{D}_{\perp}^{2}\right)+2 i P_{i}\left(\bar{\psi}\left(z_{1 *}\right) \overleftarrow{D}^{i}\right)\right]\right. \\
& \quad \times\left[z_{1 *}, x_{*}\right] t^{a} p_{1}\left[x_{*}, y_{*}\right] t^{b}\left[y_{*}, z_{2 *}\right] \psi\left(z_{2 *}\right)\left[z_{2 *}, y_{*}\right]+\bar{\psi}\left(z_{1 *}\right)\left[z_{1 *}, x_{*}\right] t^{a} p_{1}\left[x_{*}, y_{*}\right] t^{b}\left[y_{*}, z_{2 *}\right] \\
& \left.\quad \times\left[\psi\left(z_{2 *}\right) P_{\perp}^{2}-2 i\left(D^{i} \psi\left(z_{2 *}\right)\right) P_{i}-\left(D_{\perp}^{2} \psi\left(z_{2 *}\right)\right)\right]\right) e^{i \frac{\hat{p}_{\perp}^{2}}{\alpha s} y_{*}\left|y_{\perp}\right\rangle}
\end{aligned}
$$

where we used $e^{i \frac{\hat{p}_{\perp}^{2}}{\alpha s} z_{1 *}} \psi e^{-i \frac{\hat{p}_{\perp}^{2}}{\alpha s} z_{1 *}}=\psi+O\left(\lambda^{-1}\right)$ and similarly for $\bar{\psi}$.

The other terms in eq. (F.13) will contribute as sub-eikonal terms so, the final result is (including also the cross diagram)

$$
\begin{aligned}
&\left\langle A_{\mu}^{a}(x) A_{\nu}^{b}(y)\right\rangle_{\psi \bar{\psi}}=\left[-\int_{0}^{+\infty} \frac{d \alpha}{2 \alpha} \theta\left(x_{*}-y_{*}\right)+\int_{-\infty}^{0} \frac{d \alpha}{2 \alpha} \theta\left(y_{*}-x_{*}\right)\right] e^{-i \alpha\left(x_{\bullet}-y_{\bullet}\right)} \\
& \times\left\{\frac{4}{s^{2}} p_{2 \mu} p_{2 \nu}\left\langle x_{\perp}\left|e^{-i \frac{\hat{p}_{\perp}^{2}}{\alpha s} x_{*}} \frac{i g}{2 \alpha^{2}} \int_{y_{*}}^{x_{*}} d \frac{2}{s} z_{1 *}\left[x_{*}, z_{1 *}\right]^{a c}\left(\left(D^{i} F_{i \bullet}\right)+\frac{2}{s}\left(D_{\bullet} F_{*}\right)\right)^{c d}\left[z_{1 *}, y_{*}\right]^{d b} e^{i \frac{\hat{p}_{\perp}^{2}}{\alpha s} y_{*}}\right| y_{\perp}\right\rangle\right. \\
&+\left\langle x_{\perp}\right| e^{-i \frac{\hat{p}_{\perp}^{2}}{\alpha s} x_{*}}\left[\mathfrak{Q}_{1 \mu \nu}^{a b}\left(x_{*}, y_{*} ; p_{\perp}\right)+\mathfrak{Q}_{2 \mu \nu}^{a b}\left(x_{*}, y_{*} ; p_{\perp}\right)+\mathfrak{Q}_{3 \mu \nu}^{a b}\left(x_{*}, y_{*} ; p_{\perp}\right)\right. \\
&\left.+\mathfrak{Q}_{4 \mu \nu}^{a b}\left(x_{*}, y_{*} ; p_{\perp}\right)+\mathfrak{Q}_{5 \mu \nu}^{a b}\left(x_{*}, y_{*} ; p_{\perp}\right)\right] e^{i \frac{\hat{p}_{\perp}^{2}}{\alpha s} y_{*}}\left|y_{\perp}\right\rangle \\
&+\left\langle y_{\perp}\right| e^{-i \frac{\hat{p}_{\perp}^{2}}{\alpha s} y_{*}}[ {\left[\mathfrak{Q}_{2 \nu \mu}^{b a}\left(y_{*}, x_{*} ; p_{\perp}\right)+\mathfrak{Q}_{3 \nu \mu}^{b a}\left(y_{*}, x_{*} ; p_{\perp}\right)\right.} \\
&+\left.\left.\mathfrak{Q}_{4 \nu \mu}^{b a}\left(y_{*}, x_{*} ; p_{\perp}\right)+\mathfrak{Q}_{5 \nu \mu}^{b a}\left(y_{*}, x_{*} ; p_{\perp}\right)\right] e^{i \frac{\hat{p}_{\perp}^{2}}{\alpha s} x_{*}}\left|x_{\perp}\right\rangle+O\left(\lambda^{-2}\right)\right\}
\end{aligned}
$$

where we define

$$
\begin{aligned}
& \mathfrak{Q}_{1 \mu \nu}^{a b}\left(x_{*}, y_{*} ; p_{\perp}\right) \\
& =\frac{4}{s^{2}} p_{2 \mu} p_{2 \nu} \frac{i g}{2 \alpha^{2}} \int_{y_{*}}^{x_{*}} d \frac{2}{s} z_{1 *}\left[\frac{z_{1 *}}{\alpha s}\left\{P^{k},\left[x_{*}, z_{1 *}\right]^{a c}\left(\left(D_{k} D^{i} F_{i \bullet}\right)+\frac{2}{s}\left(D_{k} D \bullet F_{*}\right)\right)^{c d}\left[z_{1 *}, y_{*}\right]^{d b}\right\}\right. \\
& \quad+\left(\frac{g}{2 \alpha}\right) \int_{z_{1 *}}^{x_{*}} d \frac{2}{s} \omega_{*} \frac{2}{s} z_{1 *}\left(\left(\left[x_{*}, \omega_{*}\right] F_{k \bullet}\left[\omega_{*}, z_{1 *}\right]\right)^{a c}\left(\left(D^{k} D^{i} F_{i \bullet}\right)+\frac{2}{s}\left(D^{k} D_{\bullet} F_{* \bullet}\right)\right)^{c d}\left[z_{1 *}, y_{*}\right]^{d b}\right. \\
& \left.\left.\quad-\left[x_{*}, \omega_{*}\right]^{a c}\left(\left(D^{k} D^{i} F_{i \bullet}\right)+\frac{2}{s}\left(D^{k} D_{\bullet} F_{* \bullet}\right)\right)^{c d}\left(\left[\omega_{*}, z_{1 *}\right] F_{k \bullet}\left[z_{1 *}, y_{*}\right]\right)^{d b}\right)\right]
\end{aligned}
$$




$$
\begin{aligned}
& \mathfrak{Q}_{2 \mu \nu}^{a b}\left(x_{*}, y_{*} ; p_{\perp}\right) \\
& =\frac{g^{2}}{\alpha} \frac{4}{s^{2}} p_{2 \mu} p_{2 \nu}\left\{\frac { 1 } { 8 \alpha ^ { 2 } } \int _ { x _ { * } } ^ { y _ { * } } d \frac { 2 } { s } z _ { 1 * } \int _ { y _ { * } } ^ { z _ { 1 * } } d \frac { 2 } { s } z _ { 2 * } \left(\left[P_{\perp}^{2} \bar{\psi}\left(z_{1 *}\right)-\left(\bar{\psi}\left(z_{1 *}\right) \overleftarrow{D}_{\perp}^{2}\right)+2 i P_{i}\left(\bar{\psi}\left(z_{1 *}\right) \overleftarrow{D}^{i}\right)\right]\right.\right. \\
& \times\left[z_{1 *}, x_{*}\right] t^{a} \not p_{1}\left[x_{*}, y_{*}\right] t^{b}\left[y_{*}, z_{2 *}\right] \psi\left(z_{2 *}\right)\left[z_{2 *}, y_{*}\right]+\bar{\psi}\left(z_{1 *}\right)\left[z_{1 *}, x_{*}\right] t^{a} p_{1}\left[x_{*}, y_{*}\right] t^{b}\left[y_{*}, z_{2 *}\right] \\
& \left.\times\left[\psi\left(z_{2 *}\right) P_{\perp}^{2}-2 i\left(D^{i} \psi\left(z_{2 *}\right)\right) P_{i}-\left(D_{\perp}^{2} \psi\left(z_{2 *}\right)\right)\right]\right) \\
& +\left[\frac{1}{2} \int_{y_{*}}^{x_{*}} d \frac{2}{s} z_{1 *} \int_{y_{*}}^{z_{1 *}} d \frac{2}{s} \omega_{1 *} \int_{y_{*}}^{\omega_{1 *}} d \frac{2}{s} z_{2 *}\left(P_{j} \bar{\psi}\left(z_{1 *}\right)+\left(-i \bar{\psi}\left(z_{1 *}\right) \overleftarrow{D}_{j}\right)\right)\right. \\
& \times\left[z_{1 *}, x_{*}\right] t^{a}\left[x_{*}, \omega_{1 *}\right] g F_{i \bullet}\left[\omega_{1 *}, y_{*}\right] t^{b}\left[y_{*}, z_{2 *}\right] \\
& +\int_{y_{*}}^{x_{*}} d \frac{2}{s} z_{1 *} \int_{y_{*}}^{z_{1 *}} d \frac{2}{s} z_{2 *} \int_{y_{*}}^{z_{2 *}} d \frac{2}{s} \omega_{1 *}\left(P_{j} \bar{\psi}\left(z_{1 *}\right)+\left(-i \bar{\psi}\left(z_{1 *}\right) \overleftarrow{D}_{j}\right)\right) \\
& \times\left(\left[z_{1 *}, x_{*}\right] t^{a}\left[x_{*}, \omega_{1 *}\right] g F_{i \bullet}\left[\omega_{1 *}, y_{*}\right] t^{b}\left[y_{*}, z_{2 *}\right]\right. \\
& \left.\left.-\left[z_{1 *}, x_{*}\right] t^{a}\left[x_{*}, y_{*}\right] t^{b}\left[y_{*}, \omega_{1 *}\right] g F_{i \bullet}\left[\omega_{1 *}, z_{2 *}\right]\right)\right] \gamma^{j} \not p_{1} \gamma^{i} \psi\left(z_{2 *}\right) \\
& -\left[\frac{1}{2} \int_{y_{*}}^{x_{*}} d \frac{2}{s} z_{1 *} \int_{y_{*}}^{z_{1 *}} d \frac{2}{s} \omega_{1 *} \int_{y_{*}}^{\omega_{1 *}} d \frac{2}{s} z_{2 *} \bar{\psi}\left(z_{1 *}\right)\left[z_{1 *}, x_{*}\right] t^{a}\left[x_{*}, \omega_{1 *}\right] g F_{j \bullet}\left[\omega_{1 *}, y_{*}\right] t^{b}\left[y_{*}, z_{2 *}\right]\right. \\
& +\int_{y_{*}}^{x_{*}} d \frac{2}{s} \omega_{1 *} \int_{y_{*}}^{\omega_{1 *}} d \frac{2}{s} z_{1 *} \int_{y_{*}}^{z_{1 *}} d \frac{2}{s} z_{2 *} \bar{\psi}\left(z_{1 *}\right)\left(\left[z_{1 *}, x_{*}\right] t^{a}\left[x_{*}, \omega_{1 *}\right] g F_{j \bullet}\left[\omega_{1 *}, y_{*}\right] t^{b}\left[y_{*}, z_{2 *}\right]\right. \\
& \left.\left.-\left[z_{1 *}, \omega_{1 *}\right] g F_{j \bullet}\left[\omega_{1 *}, x_{*}\right] t^{a}\left[x_{*}, y_{*}\right] t^{b}\left[y_{*}, z_{2 *}\right]\right)\right] \gamma^{j} \not p_{1} \gamma^{i}\left(\left(i D_{i} \psi\left(z_{2 *}\right)\right)+\psi\left(z_{2 *}\right) P_{i}\right) \\
& +\left[\int_{y_{*}}^{x_{*}} d \frac{2}{s} \omega_{1 *} \int_{y_{*}}^{\omega_{1 *}} d \frac{2}{s} z_{1 *} \int_{y_{*}}^{z_{1 *}} d \frac{2}{s} z_{2 *} \int_{y_{*}}^{z_{2 *}} d \frac{2}{s} \omega_{2 *} \bar{\psi}\left(z_{1 *}\right)\right. \\
& \times\left(\left[z_{1 *}, x_{*}\right] t^{a}\left[x_{*}, \omega_{1 *}\right] g F_{j \bullet}\left[\omega_{1 *}, \omega_{2 *}\right] g F_{i \bullet}\left[\omega_{2 *}, y_{*}\right] t^{b}\left[y_{*}, z_{2 *}\right]\right. \\
& -\left[z_{1 *}, \omega_{1 *}\right] g F_{j \bullet}\left[\omega_{1 *}, x_{*}\right] t^{a}\left[x_{*}, \omega_{2 *}\right] g F_{i \bullet}\left[\omega_{2 *}, y_{*}\right] t^{b}\left[y_{*}, z_{2 *}\right] \\
& +\left[z_{1 *}, \omega_{1 *}\right] g F_{j \bullet}\left[\omega_{1 *}, x_{*}\right] t^{a}\left[x_{*}, y_{*}\right] t^{b}\left[y_{*}, \omega_{2 *}\right] g F_{i \bullet}\left[\omega_{2 *}, z_{2 *}\right] \\
& \left.-\left[z_{1 *}, x_{*}\right] t^{a}\left[x_{*}, \omega_{1 *}\right] g F_{j \bullet}\left[\omega_{1 *}, y_{*}\right] t^{b}\left[y_{*}, \omega_{2 *}\right] g F_{i \bullet}\left[\omega_{2 *}, z_{2 *}\right]\right) \\
& +\frac{1}{2} \int_{y_{*}}^{x_{*}} d \frac{2}{s} \omega_{1 *} \int_{y_{*}}^{\omega_{1 *}} d \frac{2}{s} z_{1 *} \int_{y_{*}}^{z_{1 *}} d \frac{2}{s} \omega_{2 *} \int_{y_{*}}^{\omega_{2 *}} d \frac{2}{s} z_{2 *} \bar{\psi}\left(z_{1 *}\right) \\
& \times\left(\left[z_{1 *}, x_{*}\right] t^{a}\left[x_{*}, \omega_{1 *}\right] g F_{j \bullet}\left[\omega_{1 *}, \omega_{2 *}\right] g F_{i \bullet}\left[\omega_{2 *}, y_{*}\right] t^{b}\left[y_{*}, z_{2 *}\right]\right. \\
& \left.-\left[z_{1 *}, \omega_{1 *}\right] g F_{j \bullet}\left[\omega_{1 *}, x_{*}\right] t^{a}\left[x_{*}, \omega_{2 *}\right] g F_{i \bullet}\left[\omega_{2 *}, y_{*}\right] t^{b}\left[y_{*}, z_{2 *}\right]\right) \\
& +\frac{1}{2} \int_{y_{*}}^{x_{*}} d \frac{2}{s} z_{1 *} \int_{y_{*}}^{z_{1 *}} d \frac{2}{s} \omega_{1 *} \int_{y_{*}}^{\omega_{1 *}} d \frac{2}{s} z_{2 *} \int_{y_{*}}^{z_{2 *}} d \frac{2}{s} \omega_{2 *} \bar{\psi}\left(z_{1 *}\right) \\
& \times\left(\left[z_{1 *}, x_{*}\right] t^{a}\left[x_{*}, \omega_{1 *}\right] g F_{j \bullet}\left[\omega_{1 *}, \omega_{2 *}\right] g F_{i \bullet}\left[\omega_{2 *}, y_{*}\right] t^{b}\left[y_{*}, z_{2 *}\right]\right. \\
& \left.\left.\left.-\left[z_{1 *}, x_{*}\right] t^{a}\left[x_{*}, \omega_{1 *}\right] g F_{j \bullet}\left[\omega_{1 *}, y_{*}\right] t^{b}\left[y_{*}, \omega_{2 *}\right] g F_{i \bullet}\left[\omega_{2 *}, z_{2 *}\right]\right)\right] \gamma^{j} \not p_{1} \gamma^{i} \psi\left(z_{2 *}\right)\right\}
\end{aligned}
$$




$$
\begin{aligned}
\mathfrak{Q}_{3 \mu \nu}^{a b}\left(x_{*}, y_{*} ; p_{\perp}\right)= & \frac{g^{2}}{4 \alpha} \int_{y_{*}}^{x_{*}} d \frac{2}{s} z_{1 *} \int_{y_{*}}^{z_{1 *}} d \frac{2}{s} z_{2 *} \bar{\psi}\left(z_{1 *}\right) \gamma_{\mu}^{\perp} \not p_{1} \gamma_{\nu}^{\perp}\left[z_{1 *}, x_{*}\right] t^{a}\left[x_{*}, y_{*}\right] t^{b}\left[y_{*}, z_{2 *}\right] \psi\left(z_{2 *}\right) \\
\mathfrak{Q}_{4 \mu \nu}^{a b}\left(x_{*}, y_{*} ; p_{\perp}\right)= & \frac{g^{2}}{8 \alpha^{2}} \int_{y_{*}}^{x_{*}} d \frac{2}{s} z_{1 *} \int_{y_{*}}^{z_{1 *}} d \frac{2}{s} z_{2 *} \bar{\psi}\left(z_{1 *}\right)\left(\frac{2}{s} p_{2 \mu} \gamma^{j} \not p_{1} \gamma_{\nu}^{\perp}-\frac{2}{s} p_{2 \nu} \gamma_{\mu}^{\perp} \not p_{1} \gamma^{j}\right) \\
& \times\left[\int_{y_{*}}^{x_{*}} d \frac{2}{s} \omega_{*}\left[z_{1 *}, x_{*}\right] t^{a}\left[x_{*}, \omega_{*}\right] g F_{j \bullet}\left[\omega_{*}, y_{*}\right] t^{b}\left[y_{*}, z_{2 *}\right]\right. \\
& -\int_{z_{1 *}}^{x_{*}} d \frac{2}{s} \omega_{*}\left[z_{1 *}, \omega_{*}\right] g F_{j \bullet}\left[\omega_{*}, x_{*}\right] t^{a}\left[x_{*}, y_{*}\right] t^{b}\left[y_{*}, z_{2 *}\right] \\
& -\int_{y_{*}}^{z_{2 *}} d \frac{2}{s} \omega_{*}\left[z_{1 *}, x_{*}\right] t^{a}\left[x_{*}, y_{*}\right] t^{b}\left[y_{*}, \omega_{*}\right] g F_{j \bullet}\left[\omega_{*}, z_{2 *}\right] \psi \psi\left(z_{2 *}\right) \\
\mathfrak{Q}_{5 \mu \nu}^{a b}\left(x_{*}, y_{*} ; p_{\perp}\right)= & \frac{g^{2}}{8 \alpha^{2}} \int_{y_{*}}^{x_{*}} d \frac{2}{s} z_{1 *} \int_{y_{*}}^{z_{1 *}} d \frac{2}{s} z_{2 *}\left[\bar{\psi}\left(z_{1 *}\right)\left[z_{1 *}, x_{*}\right] t^{a}\left[x_{*}, y_{*}\right] t^{b}\left[y_{*}, z_{2 *}\right]\right. \\
& \times\left(\frac{2}{s} p_{2 \mu} \gamma^{j} p_{1} \gamma_{\nu}^{\perp}+\frac{2}{s} p_{2 \nu} \gamma_{\mu}^{\perp} \not p_{1} \gamma^{j}\right)\left(\left(i D_{j} \psi\left(z_{2 *}\right)\right)+\psi\left(z_{2 *}\right) P_{j}\right) \\
& -\left(P_{j} \bar{\psi}\left(z_{1 *}\right)+\left(-i \bar{\psi}\left(z_{1 *}\right) \overleftarrow{D_{j}}\right)\right)\left(\frac{2}{s} p_{2 \mu} \gamma^{j} \not p_{1} \gamma_{\nu}^{\perp}+\frac{2}{s} p_{2 \nu} \gamma_{\mu}^{\perp} p_{1} \gamma^{j}\right) \\
& \left.\times\left[z_{1 *}, x_{*}\right] t^{a}\left[x_{*}, y_{*}\right] t^{b}\left[y_{*}, z_{2 *}\right] \psi\left(z_{2 *}\right)\right]
\end{aligned}
$$

Notice that in $\mathfrak{Q}_{4 \mu \nu}^{a b}$ we can use

$$
\begin{aligned}
-i \mathfrak{D}_{j}\left(\left[z_{1 *}, x_{*}\right] t^{a}\left[x_{*}, y_{*}\right] t^{b}\left[y_{*}, z_{2 *}\right]\right)= & \int_{y_{*}}^{x_{*}} d \frac{2}{s} \omega_{*}\left[z_{1 *}, x_{*}\right] t^{a}\left[x_{*}, \omega_{*}\right] g F_{j \bullet}\left[\omega_{*}, y_{*}\right] t^{b}\left[y_{*}, z_{2 *}\right] \\
& -\int_{z_{1 *}}^{x_{*}} d \frac{2}{s} \omega_{*}\left[z_{1 *}, \omega_{*}\right] g F_{j \bullet}\left[\omega_{*}, x_{*}\right] t^{a}\left[x_{*}, y_{*}\right] t^{b}\left[y_{*}, z_{2 *}\right] \\
& -\int_{y_{*}}^{z_{2 *}} \frac{2}{s} \omega_{*}\left[z_{1 *}, x_{*}\right] t^{a}\left[x_{*}, y_{*}\right] t^{b}\left[y_{*}, \omega_{*}\right] g F_{j \bullet}\left[\omega_{*}, z_{2 *}\right]
\end{aligned}
$$

and $\mathfrak{Q}_{1 \mu \nu}^{a b}\left(x_{*}, y_{*} ; p_{\perp}\right)$ includes both diagram and cross diagram, so we did not need to include $\mathfrak{Q}_{1 \nu \mu}^{b a}\left(y_{*}, x_{*} ; p_{\perp}\right)$ in eq. (F.21).

Equation (F.21) is the final result for the gluon propagator in the background-Feynman gauge in the quark and anti-quark external fields.

\section{F.3 Summing up the gluon and quark anti-quark external fields contributions}

We can now sum up the contribution due to the gluon external field eq. (F.3) and the quark and anti-quark external field eq. (F.21) and obtain the final expression of the gluon propagator in the Background-Feynman gauge up to sub-eikonal terms $(i$. e. terms that 
scale as $\left.\lambda^{-1}\right)$

$$
\begin{aligned}
& \left\langle A_{\mu}^{a}(x) A_{\nu}^{b}(y)\right\rangle=\left[-\int_{0}^{+\infty} \frac{d \alpha}{2 \alpha} \theta\left(x_{*}-y_{*}\right)+\int_{-\infty}^{0} \frac{d \alpha}{2 \alpha} \theta\left(y_{*}-x_{*}\right)\right] e^{-i \alpha\left(x_{\bullet}-y_{\bullet}\right)} \\
& \times\left(\langle x _ { \perp } | e ^ { - i \frac { \hat { p } _ { \perp } ^ { 2 } } { \alpha s } x _ { * } } \left\{g_{\mu \nu}\left[x_{*}, y_{*}\right]^{a b}+\frac{2 g}{\alpha s} \int_{y_{*}}^{x_{*}} d \frac{2}{s} \omega_{*}\left[\left(p_{2 \mu} \delta_{\nu}^{j}-p_{2 \nu} \delta_{\mu}^{j}\right)\left[x_{*}, \omega_{*}\right] F_{j \bullet}\left[\omega_{*}, y_{*}\right]\right.\right.\right. \\
& +\frac{1}{\alpha s} p_{2 \mu} p_{2 \nu}\left(\left[x_{*}, \omega_{*}\right]\left(\left(i D^{i} F_{i \bullet}\right)+\frac{2}{s}\left(i D_{\bullet} F_{* \bullet}\right)\right)\left[\omega_{*}, y_{*}\right]\right. \\
& \left.\left.-2 g \int_{\omega_{*}}^{x_{*}} d \frac{2}{s} \omega_{*}^{\prime}\left[x_{*}, \omega_{*}^{\prime}\right] F^{i} \bullet\left[\omega_{*}^{\prime}, \omega_{*}\right] F_{i \bullet}\left[\omega_{*}, y_{*}\right]\right)\right]^{a b} \\
& +\mathfrak{B}_{1 \mu \nu}^{a b}\left(x_{*}, y_{*} ; p_{\perp}\right)+\mathfrak{B}_{2 \mu \nu}^{a b}\left(x_{*}, y_{*} ; p_{\perp}\right)+\mathfrak{B}_{3 \mu \nu}^{a b}\left(x_{*}, y_{*} ; p_{\perp}\right)+\mathfrak{B}_{4 \mu \nu}^{a b}\left(x_{*}, y_{*} ; p_{\perp}\right) \\
& +\mathfrak{B}_{5 \mu \nu}^{a b}\left(x_{*}, y_{*} ; p_{\perp}\right)+\mathfrak{B}_{6 \mu \nu}^{a b}\left(x_{*}, y_{*} ; p_{\perp}\right)+\mathfrak{Q}_{1 \mu \nu}^{a b}\left(x_{*}, y_{*} ; p_{\perp}\right)+\mathfrak{Q}_{2 \mu \nu}^{a b}\left(x_{*}, y_{*} ; p_{\perp}\right) \\
& \left.+\mathfrak{Q}_{3 \mu \nu}^{a b}\left(x_{*}, y_{*} ; p_{\perp}\right)+\mathfrak{Q}_{4 \mu \nu}^{a b}\left(x_{*}, y_{*} ; p_{\perp}\right)+\mathfrak{Q}_{5 \mu \nu}^{a b}\left(x_{*}, y_{*} ; p_{\perp}\right)\right\} e^{i \frac{\hat{p}_{\perp}^{2}}{\alpha s} y_{*}}\left|y_{\perp}\right\rangle \\
& +\left\langle y_{\perp}\right| e^{-i \frac{\hat{p}_{\perp}^{2}}{\alpha s} y_{*}}\left[\mathfrak{Q}_{2 \nu \mu}^{b a}\left(y_{*}, x_{*} ; p_{\perp}\right)+\mathfrak{Q}_{3 \nu \mu}^{b a}\left(y_{*}, x_{*} ; p_{\perp}\right)\right. \\
& \left.\left.+\mathfrak{Q}_{4 \nu \mu}^{b a}\left(y_{*}, x_{*} ; p_{\perp}\right)+\mathfrak{Q}_{5 \nu \mu}^{b a}\left(y_{*}, x_{*} ; p_{\perp}\right)\right] e^{i \frac{\hat{p}_{\perp}^{2}}{\alpha s} x_{*}}\left|x_{\perp}\right\rangle\right)+O\left(\lambda^{-2}\right)
\end{aligned}
$$

Equation (F.28) is the gluon propagator in the background-Feynman gauge up to subeikonal corrections. All the sub-eikonal corrections, which scale as $\lambda^{-1}$ under the longitudinal boost, are enclosed in the operators $\mathfrak{B}_{1 \mu \nu}^{a b}, \mathfrak{B}_{2 \mu \nu}^{a b}, \mathfrak{B}_{3 \mu \nu}^{a b}, \mathfrak{B}_{4 \mu \nu}^{a b}, \mathfrak{B}_{5 \mu \nu}^{a b}, \mathfrak{B}_{6 \mu \nu}^{a b}$ and $\mathfrak{Q}_{1 \mu \nu}^{a b}$, $\mathfrak{Q}_{2 \mu \nu}^{a b}, \mathfrak{Q}_{3 \mu \nu}^{a b}, \mathfrak{Q}_{4 \mu \nu}^{a b}, \mathfrak{Q}_{5 \mu \nu}^{a b}$.

Open Access. This article is distributed under the terms of the Creative Commons Attribution License (CC-BY 4.0), which permits any use, distribution and reproduction in any medium, provided the original author(s) and source are credited.

\section{References}

[1] I. Balitsky, High-energy QCD and Wilson lines, hep-ph/0101042 [INSPIRE].

[2] I. Balitsky, Operator expansion for high-energy scattering, Nucl. Phys. B 463 (1996) 99 [hep-ph/9509348] [INSPIRE].

[3] J. Jalilian-Marian, A. Kovner and H. Weigert, The Wilson renormalization group for low $x$ physics: Gluon evolution at finite parton density, Phys. Rev. D 59 (1998) 014015 [hep-ph/9709432] [INSPIRE].

[4] J. Jalilian-Marian, A. Kovner, A. Leonidov and H. Weigert, The Wilson renormalization group for low x physics: Towards the high density regime, Phys. Rev. D 59 (1998) 014014 [hep-ph/9706377] [INSPIRE].

[5] E. Iancu, A. Leonidov and L.D. McLerran, The Renormalization group equation for the color glass condensate, Phys. Lett. B 510 (2001) 133 [hep-ph/0102009] [INSPIRE].

[6] E. Iancu, A. Leonidov and L.D. McLerran, Nonlinear gluon evolution in the color glass condensate. 1., Nucl. Phys. A 692 (2001) 583 [hep-ph/0011241] [InSPIRE]. 
[7] Y.V. Kovchegov, Small-x $F_{2}$ structure function of a nucleus including multiple Pomeron exchanges, Phys. Rev. D 60 (1999) 034008 [hep-ph/9901281] [INSPIRE].

[8] Y.V. Kovchegov, Unitarization of the BFKL Pomeron on a nucleus, Phys. Rev. D 61 (2000) 074018 [hep-ph/9905214] [INSPIRE].

[9] E.A. Kuraev, L.N. Lipatov and V.S. Fadin, The Pomeranchuk Singularity in Nonabelian Gauge Theories, Sov. Phys. JETP 45 (1977) 199 [InSPIRE].

[10] I.I. Balitsky and L.N. Lipatov, The Pomeranchuk Singularity in Quantum Chromodynamics, Sov. J. Nucl. Phys. 28 (1978) 822 [inSPIRE].

[11] Y.V. Kovchegov and E. Levin, Quantum chromodynamics at high energy, Camb. Monogr. Part. Phys. Nucl. Phys. Cosmol. 33 (2012) [inSPIRE].

[12] I. Balitsky and G.A. Chirilli, High-energy amplitudes in $N=4 S Y M$ in the next-to-leading order, Phys. Lett. B 687 (2010) 204 [arXiv:0911.5192] [INSPIRE].

[13] I. Balitsky and G.A. Chirilli, Photon impact factor in the next-to-leading order, Phys. Rev. D 83 (2011) 031502 [arXiv: 1009.4729] [INSPIRE].

[14] I. Balitsky and G.A. Chirilli, Photon impact factor and $k_{T}$-factorization for DIS in the next-to-leading order, Phys. Rev. D 87 (2013) 014013 [arXiv:1207.3844] [INSPIRE].

[15] G.A. Chirilli, B.-W. Xiao and F. Yuan, One-loop Factorization for Inclusive Hadron Production in pA Collisions in the Saturation Formalism, Phys. Rev. Lett. 108 (2012) 122301 [arXiv: 1112.1061] [INSPIRE].

[16] G.A. Chirilli, B.-W. Xiao and F. Yuan, Inclusive Hadron Productions in pA Collisions, Phys. Rev. D 86 (2012) 054005 [arXiv: 1203.6139] [INSPIRE].

[17] G.A. Chirilli and Y.V. Kovchegov, $\gamma^{*} \gamma^{*}$ Cross Section at NLO and Properties of the BFKL Evolution at Higher Orders, JHEP 05 (2014) 099 [Erratum ibid. 08 (2015) 075] [arXiv: 1403.3384] [INSPIRE].

[18] D. Boer et al., Gluons and the quark sea at high energies: Distributions, polarization, tomography, arXiv:1108.1713 [INSPIRE].

[19] A. Accardi et al., Electron Ion Collider: The Next QCD Frontier. Understanding the glue that binds us all, Eur. Phys. J. A 52 (2016) 268 [arXiv:1212.1701] [INSPIRE].

[20] E.C. Aschenauer, R. Sassot and M. Stratmann, Unveiling the Proton Spin Decomposition at a Future Electron-Ion Collider, Phys. Rev. D 92 (2015) 094030 [arXiv: 1509.06489] [InSPIRE].

[21] J.D. Bjorken, J.B. Kogut and D.E. Soper, Quantum Electrodynamics at Infinite Momentum: Scattering from an External Field, Phys. Rev. D 3 (1971) 1382 [INSPIRE].

[22] G. 't Hooft, Graviton Dominance in Ultrahigh-Energy Scattering, Phys. Lett. B 198 (1987) 61 [INSPIRE].

[23] J.C. Collins, D.E. Soper and G.F. Sterman, Heavy Particle Production in High-Energy Hadron Collisions, Nucl. Phys. B 263 (1986) 37 [INSPIRE].

[24] H. Cheng and T.T. Wu, Expanding Protons: Scattering At High-energies, Cambridge, U.S.A., MIT-PR. (1987) [INSPIRE].

[25] O. Nachtmann, Considerations concerning diffraction scattering in quantum chromodynamics, Annals Phys. 209 (1991) 436 [INSPIRE].

[26] I. Balitsky and A. Tarasov, Rapidity evolution of gluon TMD from low to moderate $x$, JHEP 10 (2015) 017 [arXiv: 1505.02151] [INSPIRE]. 
[27] I. Balitsky and A. Tarasov, Gluon TMD in particle production from low to moderate $x$, JHEP 06 (2016) 164 [arXiv: 1603.06548] [INSPIRE].

[28] Y.V. Kovchegov, D. Pitonyak and M.D. Sievert, Helicity Evolution at Small-x, JHEP 01 (2016) 072 [Erratum ibid. 10 (2016) 148] [arXiv:1511.06737] [INSPIRE].

[29] Y.V. Kovchegov, D. Pitonyak and M.D. Sievert, Helicity Evolution at Small x: Flavor Singlet and Non-Singlet Observables, Phys. Rev. D 95 (2017) 014033 [arXiv: 1610.06197] [INSPIRE].

[30] Y.V. Kovchegov, D. Pitonyak and M.D. Sievert, Small-x Asymptotics of the Quark Helicity Distribution: Analytic Results, Phys. Lett. B 772 (2017) 136 [arXiv:1703. 05809] [INSPIRE].

[31] Y.V. Kovchegov, D. Pitonyak and M.D. Sievert, Small-x Asymptotics of the Gluon Helicity Distribution, JHEP 10 (2017) 198 [arXiv:1706.04236] [INSPIRE].

[32] J. Bartels, B.I. Ermolaev and M.G. Ryskin, Nonsinglet contributions to the structure function g1 at small $x$, Z. Phys. C 70 (1996) 273 [hep-ph/9507271] [INSPIRE].

[33] J. Bartels, B.I. Ermolaev and M.G. Ryskin, Flavor singlet contribution to the structure function $g_{1}$ at small $x, Z$. Phys. C 72 (1996) 627 [hep-ph/9603204] [INSPIRE].

[34] V.G. Gorshkov, V.N. Gribov, L.N. Lipatov and G.V. Frolov, Doubly logarithmic asymptotic behavior in quantum electrodynamics, Sov. J. Nucl. Phys. 6 (1968) 95 [INSPIRE].

[35] T. Altinoluk, N. Armesto, G. Beuf, M. Martínez and C.A. Salgado, Next-to-eikonal corrections in the CGC: gluon production and spin asymmetries in pA collisions, JHEP 07 (2014) 068 [arXiv: 1404.2219] [InSPIRE].

[36] E. Laenen, G. Stavenga and C.D. White, Path integral approach to eikonal and next-to-eikonal exponentiation, JHEP 03 (2009) 054 [arXiv: 0811.2067] [INSPIRE].

[37] E. Laenen, L. Magnea and G. Stavenga, On next-to-eikonal corrections to threshold resummation for the Drell-Yan and DIS cross sections, Phys. Lett. B 669 (2008) 173 [arXiv: 0807.4412] [INSPIRE].

[38] E. Laenen, L. Magnea, G. Stavenga and C.D. White, Next-to-eikonal corrections to soft gluon radiation: a diagrammatic approach, JHEP 01 (2011) 141 [arXiv:1010.1860] [INSPIRE].

[39] G.A. Chirilli, Y.V. Kovchegov and D.E. Wertepny, Regularization of the Light-Cone Gauge Gluon Propagator Singularities Using Sub-Gauge Conditions, JHEP 12 (2015) 138 [arXiv: 1508.07962] [INSPIRE].

[40] I.I. Balitsky and V.M. Braun, Evolution Equations for QCD String Operators, Nucl. Phys. B 311 (1989) 541 [INSPIRE].

[41] V.M. Braun, G.P. Korchemsky and A.N. Manashov, Evolution of twist - three parton distributions in QCD beyond the large $N_{c}$ limit, Phys. Lett. B 476 (2000) 455 [hep-ph/0001130] [INSPIRE].

[42] V.M. Braun, A.N. Manashov and J. Rohrwild, Baryon Operators of Higher Twist in QCD and Nucleon Distribution Amplitudes, Nucl. Phys. B 807 (2009) 89 [arXiv:0806.2531] [INSPIRE].

[43] V.M. Braun, A.N. Manashov and B. Pirnay, Scale dependence of twist-three contributions to single spin asymmetries, Phys. Rev. D 80 (2009) 114002 [Erratum ibid. D 86 (2012) 119902] [arXiv:0909.3410] [INSPIRE].

[44] V.M. Braun, Y. Ji and A.N. Manashov, Higher-twist B-meson Distribution Amplitudes in HQET, JHEP 05 (2017) 022 [arXiv:1703.02446] [INSPIRE].

[45] J.C. Collins, Foundations of Perturbative QCD, Cambridge University Press, Cambridge U.K. (2011) [INSPIRE]. 